\title{
Industrial Wastewater Treatment Technologies For Reuse, Recycle, And Recovery: Advantages, Disadvantages, And Gaps.
}

Ricardo Mejía ( $\square$ marchenar@uninorte.edu.co)

Universidad del Norte https://orcid.org/0000-0001-9529-4029

\section{Aymer Maturana}

Universidad del Norte

Diego Gómez

Universidad del Norte

Christian Quintero

Universidad del Norte

Luis Arismendy

Universidad del Norte

Carlos Cárdenas

Universidad del Norte

\section{Research Article}

Keywords: Wastewater, Industry, water treatment, technological gaps, circular economy

Posted Date: December 17th, 2021

DOI: https://doi.org/10.21203/rs.3.rs-1147300/v1

License: (c) (1) This work is licensed under a Creative Commons Attribution 4.0 International License. Read Full License 


\section{Abstract}

To reduce demand and discharge, instead of industrial wastewater being poorly treated and disposed of, it can be recycled, reused, or recovered if it is properly managed, thus having a substantial decrease in the water requirement and environmental impacts. The challenge is to select the appropriate process or combination of processes to achieve this based on the wastewater quality. Consequently, the objective of this investigation is to review every technology from conventional through advanced, for reliable and sustainable wastewater treatment and derived sludges, focusing on advantages, disadvantages, and technical gaps for development. Even though there is a wide range of possible technologies, it was evinced that there is huge potential to exploit and make them economically and sustainably viable for waste processing and circular economy, even in the mature massively implemented wastewater treatment technologies in the industry. Overall, we identify that independently from the technology to be studied, the future investigations on every unit, especially on those not vastly implemented, should be focused on: (1) The capacity in removing selected pollutants and decreasing impurities, (2) energy efficiency, (3) environmental safety, (4) economic viability, (5) hybrid processes, and (6) sustainability by waste processing.

\section{Introduction}

Water use in the industrial sector is estimated to fluctuate between $22-80 \%$ of the total supply in developed countries, whereas in developing countries it was about $4-30 \%$, though there is enough scope for increase (Ahmetovic et al., 2017; Jana et al., 2018; Piadeh et al., 2014). With this large quantity of freshwater consumed, nearly $90 \%$ is being discharged untreated or partially treated into the ecosystems as effluents having unused raw materials and by-products in them (Mahto et al., 2021). To reduce demand and discharge, instead of industrial wastewater (IWW) being disposed of, it can be recycled, reused, or recovered if it is properly treated, thus having a substantial decrease in the water requirement and environmental impacts (Anderson, 2002; Jana et al., 2018). Even though most of the present-day operations do not intend to reuse, recover and recycle (3R) in IWW treatment unless faced with severe constraints, this has received more attention recently (Mohapatra \& Kirpalani, 2019). Nevertheless, In current practices, the industrial cycle of resources is generally a linear system since the waste is not recovered to be recirculated (Figure 1) (Piadeh et al., 2014; Ranade \& Bhandari, 2014b; Van de Worp, 2002).

Given the rapid growth of the sector, resulting in a competition for water between industry and agriculture, and the escalating cost of water thus having more efficient water use practices and the prevention of pollution, a greater emphasis on the 3R scheme of IWW and derived sludge treatment are more relevant day by day (Levine \& Asano, 2002; Ranade \& Bhandari, 2014a, 2014b). For this reason, more companies are recognizing the cost implications of water consumption and the benefits of reuse rather than disposal. The cost of recovering, recycling, and reusing water, valuable substances, and energy contained in IWW is also becoming financially more advantageous as resource costs, water supply, and effluent restrictions increase, as well as a significant reduction in the environmental impact of industry (Accepta, 1997; Levine \& Asano, 2002; Ranade \& Bhandari, 2014a; Smol et al., 2020; Strehler et al., 2018). Consequently, unit operations and processes in wastewater treatment is no longer just an approach to purify water but also an important tool 
for improving cost competitiveness, enhancing energy efficiency, creating new jobs, and increasing productivity with the fundamental goal of addressing environmental concern (Ranade \& Bhandari, 2014b; Safferman et al., 2017).

The industry has two main objectives: economic profit and continuity. From this perspective, wastewater management should contribute to these goals. In general, the current practice is to make every effort to optimize water use and emissions, rather than minimize them. In this circumstance, it is typically supposed that the only goal of IWW treatment is the removal of pollutants to such levels that it meets the norms of discharge intending to minimize the cost of treatment (Bhandari \& Ranade, 2014; Ranade \& Bhandari, 2014a; Van de Worp, 2002). Unfortunately, this approach offers a very limited and impractical scope as it limits the possibilities of the $3 R$ scheme. Water use has to be related to its quality, not just availability and economic aspects (Van de Worp, 2002).

As mentioned, the valorization of IWW is generally governed almost entirely by economics (Crini \& Lichtfouse, 2019). Increasingly stringent environmental legislation for environmental protection has created a hostile economic environment for the industry, nevertheless, greater intensity, efficiency, and diversity of treatment technologies have made effluent reuse and recovery more feasible to overcome these difficulties (Judd, 2002; Mpofu et al., 2021).

The 3R scheme can take several forms: in-process reusing of IWW with or without treatment; recycling of IWW, related to the reclamation of water for potable usage by replacing or upgrading the existing plant designed; resources recovery in wastewater, including inorganic nutrients such as nitrogen and phosphorus, organic fertilizers, biogas, biopolymers, energy, heavy metals, salts, industrial products, etc. (Judd, 2002; Smol et al., 2020). These scheme strategies in IWW aimed at closing industrial water cycles and obtaining valuable components that will in most cases require a combination of wastewater treatment operations (Figure 2). However, this is a difficult task because the wastewater contains specific pollutants, high organic matter contents, and poorly biodegradable components (Mels \& Teerikangas, 2002; Morales-arrieta et al., 2021).

For this reason, IWW treatment is a huge area covering numerous physical, chemical, and biological methods of treatment (Bhandari \& Ranade, 2014). The possible combinations of processes, but not limited, are shown in Figure 3, where generally are classified based on the degrees of treatments (Drewes et al., 2017). However, the selection of these is the major challenge and will greatly depend on the nature of the wastewater, like the quality of wastewater, nature of pollutants, and desired level of reduction/recovery of these pollutants (Ahirrao, 2014; Bhandari \& Ranade, 2014). This given that unlike urban wastewater, the quality of IWW varies enormously among themselves, even in the same category of industries, so there is no single or universal technology or sequence of these (Buccolieri et al., 2017; Crini \& Lichtfouse, 2019; Woodard \& Curran, 2006a).

The 3R scheme still requires a lot of development of technological nature (maturity, reliability, demonstration, quality control), social (public acceptance), and economic (costs, reimbursements, financial risks); the challenge is to solve them and create possibilities for sustainable development (Baawain et al., 2020; Jana et al., 2018; Mohapatra \& Kirpalani, 2019; Van de Worp, 2002). Consequently, the present state 
of the art addresses the advantages and disadvantages of unit operations and processes in the IWW treatment field, as well as those gaps and potential to exploit for the $3 \mathrm{R}$ scheme implementation in the industry sector.

\section{Iww Treatment Technologies.}

Several treatment units have been well established in chemical engineering separations and wastewater treatment in the industry (Ranade \& Bhandari, 2014a). Each one presents its limitations not only in the economic aspect but also in terms of feasibility, availability of equipment or space, removal efficiency, reliability, environmental impact, sludge and by-products production, the difficulty of operation, pretreatment requirements, and the formation of potentially toxic residues (Crini \& Lichtfouse, 2019). Despite a large number of unit treatment alternatives, only a few are common in IWW treatment, and unfortunately, as it was mentioned, their main reason for selection is the economic one (Crini \& Lichtfouse, 2019).

Following, different operations and unit processes for IWW treatment will be presented (Figure 4), which together and correct sequencing can achieve a 3R scheme technology system. In addition, various technologies for the treatment of generated sludge (Figure 5), which must be considered within the same treatment system. Additionally, We classified them into 5 different categories: Research (large scale has not been completely demonstrated), Emerging (large scale has been demonstrated but it is not fully implemented), Innovative (large scale has been demonstrated but just some degree of initial use), Established (large scale has been demonstrated and widely implemented), and Adaptive Use (established technology but they are still in research and development), which have been covered in the following sections.

\subsection{Conventional Physicochemical treatment units.}

This includes all common treatment unit operations that are not based on biological conversion, whose elimination principles are based on phase separation, molecular separation, or chemical conversion. Usually, considered in wastewater treatment as the primary process or pretreatment units, but in a 3R scheme, these could even be considered secondary or tertiary. The advantages, disadvantages, its category, common or potential industry sector, and gaps for a 3R scheme implementation in the industry are presented in Table 1. Complete technical descriptions of every technology are already covered somewhere else (Ahirrao, 2014; Bhandari \& Ranade, 2014; Kyzas, 2018; Mai et al., 2018; Mels \& Teerikangas, 2002; Nidheesh et al., 2021).

Technologies as coagulation/flocculation are one of the most widely used treatment methods and are considered well established, economical, practical, and relatively efficient in today's context (C. Zhao et al., 2021). It is typically employed as a primary or secondary treatment step, depending on the effluent's quality, either in the conventional method (chemically induced coagulation) or relatively less used in the unconventional method (electro-coagulation or Cavigulation) (Bhandari \& Ranade, 2014). Nevertheless, depending on the industry, this technology is even the key limiting factor in reusing and recovering systems (Zheng et al., 2021). 
Previously, industrial wastewater treatment plants (WWTP) mainly involved settling and filtration (Screening, Coarse media filtration to a lesser extent rapid filtration) combine with a biological process (Ranade \& Bhandari, 2014b). Nowadays, these are still extensively used in industry because of their large-scale experience and simple operation. Other technologies as flotation or chemical precipitation are common methods for IWW treatment, especially when the goal is to recover metal from it, being used the precipitation for high content of metals and flotation for low content. These have the benefits of simple process, low investment, mature technology, extensive large-scale experience, and a high degree of automation (Kyzas, 2018; J. Wang, 2018).

In addition to these treatment units, Adsorption is an effective treatment process for recalcitrant impurities which are hard to remove by biological alternatives in IWW (Mai et al., 2018). It can be considered that this process, in the same way as those already mentioned, has securely established itself in terms of commercial use and are also technologically mature, indicating their wide acceptability in the industry and remained to be one of the most promising recovery options owing to its facile implementation, low cost, high availability and high removal efficiencies even at low target metal concentrations (Dawn \& Vishwakarma, 2021; Perez et al., 2019; Ranade \& Bhandari, 2014b; J. Wang, 2018).

Other conventional unit operations as absorption, evaporation, and crystallization are generally considered in secondary and tertiary treatment stages (Ranade \& Bhandari, 2014b). However, these are not seen as unconventional or advanced unit treatment operations but are generally used in conventional recycling and reuse of wastewater systems (Ahirrao, 2014; Muhammad Yaqub \& Lee, 2019).

Based on Figure 6, adsorption processes correspond to the technology with more interest, development, and potential to exploit. The reason is that these processes are capable to produce high-quality effluents at low operational costs compared with other conventional technologies, the high adsorption potential of new material and fast adsorption of metals, toxic impurities, and persistent organic pollutants (Gupta et al., 2015; Ramírez Calderón et al., 2020). Other technologies with a special interest in IWW treatment with a likely view to a 3R scheme are ion exchange and precipitation.

\subsection{Unconventional Physicochemical treatment units.}

In the course of recent decades, conventional wastewater treatment unit operation and processes have succeeded to some extent in treating effluents for discharge purposes. Nevertheless, improvements and integration with unconventional technologies are highly necessary to consider recovery and make treated wastewater re-usable for industrial and other sector purposes (Ezugbe \& Rathilal, 2020). These unconventional physicochemical treatment units correspond to those relatively novel water treatment processes that are not based on biological conversion. Pros, drawbacks, gaps for a 3R scheme, and other relevant information are shown in Table 2, for technical descriptions of every technology the followed cited investigations are recommended (Abdullah et al., 2019; Cui et al., 2010; Devda et al., 2021; Ezugbe \& Rathilal, 2020; Judd, 2002; Poyatos et al., 2010; Saharan et al., 2014; Shrestha et al., 2021; Urbina-suarez et al., 2021; Xie et al., 2016; Yan et al., 2018b; Ye et al., 2020). 
Unconventional physicochemical treatment units such as membrane processes are often considered to be more energy-efficient, simpler to operate, and yield higher quality products and water with a low impact on the environment. Membrane technology is emerging as a promising route to recover metals, nutrients, and other components in IWW (Ali et al., 2021; Shrestha et al., 2021; Xie et al., 2016; Ye et al., 2020). However, this may not be true in all cases, and there are many limitations, especially in industrial wastewater reclamation that is strongly constrained by membrane fouling (Bhandari \& Ranade, 2014; Tong et al., 2020).

Even though membrane processes are a relatively new type of IWW treatment, several membrane processes, particularly pressure-driven membrane processes including Reverse Osmosis (RO), Nanofiltration (NF), Ultrafiltration (UF), and Microfiltration (MF), are already extensively applied on IWW treatment (Cui et al., 2010). These are commonly employed as a primary treatment as well as a fine polishing step in tertiary treatment. In fact, these are some of the processes that have the huge potential to yield pure water that may be recycled and reused (Bhandari \& Ranade, 2014).

In emerging membrane processes, such as Forward osmosis (FO), Membrane Distillation (MD) and Electrodialysis (ED), and other electrochemical membrane technologies, such as Membrane Electrolysis (ME), Electro-Electrodialysis (EED), Bipolar Membrane Electrodialysis (BMED), Membrane-assisted Electron Ionization (EDI), and Capacitive Membrane Deionization (MCDI), their selectivity is conducive to the fraction of valuable nutrient, salts, and other substances, and their energy requirements and associated costs are competitive in comparison with more conventional units, and even the already mentioned pressure-driven membrane processes (Handojo et al., 2019; Jaroszek \& Dydo, 2016a; Mejía Marchena et al., 2020; Wei et al., 2013; Xie et al., 2016). While almost all these emerging membrane technologies show huge potential, there are still disadvantages that prevent their implementation on a full industrial scale. Although many investigations have been performed on the recovery in the industry, most of these focused on synthetic matrices, which are often very distinct in quality from the real ones (Perez et al., 2019). But in general, all membrane alternatives are expected to continue growing in investigation and implementation.

Technologies as Advanced oxidation (AO) systems are not widely used in industry, this is not because of their result treated water but rather because of a general lack of awareness and understanding about the current advanced oxidation technologies and their applications to different cases (Bhuta, 2014). However, as shown in Figure 7, AO processes have a huge potential to exploit in improving the performance of IWW treatment based on the number of publications, hence, the vast implementation of these processes in the new and/or up-date WWTP in the industry is expected in the future.

Other technologies that have recently undergone a considerable increase in new developments with an important interest correspond to the electrochemical treatment. It is anticipated that electrochemical processes will be able to make an important contribution to wastewater reclamation, since these techniques can be used in a wide variety of ways, such as selective separation technology and/or degrading processes (Muddemann et al., 2019). However, most recent applications are yet at the laboratory or pilot scale stage (Muhammad Yaqub \& Lee, 2019).

\subsection{Biological treatment unit.}


In general, biological treatment is supposed to be more economical than any other type of treatment where relatively complete treatment is required and wherever it can be made to work successfully (Woodard \& Curran, 2006b). However, in most cases, industrial wastewaters contain extremely high concentrations of organic compounds, according to biological oxygen demand (BOD) and chemical oxygen demand (COD); more than ten times the concentrations in municipal wastewater. So, nutrient limitations, the presence of inhibiting or resistance to biological substances are potential problems for biological treatment of some IWW (Buccolieri et al., 2017; Ranade \& Bhandari, 2014b). A complete list of advantages, disadvantages, and gaps to overcome in the industry for each biological alternative is presented in Table 3. For technical descriptions, the cited texts are suggested (Arabi et al., 2020; Hamedi et al., 2019; Moran, 2018; Nidheesh et al., 2021; Perera et al., 2019; Singh et al., 2019; Thuptimdang et al., 2020; Urbina-suarez et al., 2021; Yildiz Sizirici, 2012).

In the same way as adsorption technologies, biological processes such as Conventional Activated Sludge (CAS), Membrane Bioreactor (MBR), and Biological Nutrient Recovery, have securely established themselves in terms of commercial use and technological maturity, indicating their wide acceptability in the industry (Figure 8). This, in comparison to mentioned processes namely membrane separation, AO processes, and coagulation that are considered technologically mature to a lesser extent and require further development for increasing their use in the industry, but expected an upward rise in the use because of the intensification of refractory pollutants (Ranade \& Bhandari, 2014b).

\subsection{Sludge treatment unit.}

IWW treatment results in a sludge that is a solid waste, these solids should be an integral part of the economics in water treatment systems, even if it is considered to be "thrown away" there is a cost involved (Fytili \& Zabaniotou, 2008; Stessel, 1996). The transition towards the implementation of 3R schemes in industrial processes often involves the assessment of only the water without taking into consideration solid wastes. However, it could be possible to simultaneously optimize the complete WWTP and consequently generate a higher economic yield and a reduced environmental impact (Elalami et al., 2019).

On the one hand, the CAS and its different configurations, being the most used technology for wastewater treatment in the world, results in the generation of a considerable amount of excess sludge that must be eliminated (Guo \& Xu, 2011; Yoshino et al., 2020). This sludge contains high volatile solids fractions and retains large amounts of water, resulting in extremely large volumes of residual solids produced and significant disposal costs (S. Pérez-Elvira et al., 2006). In fact, the treatment and disposal of this excess sludge can represent between $25-65 \%$ of the total operating cost of a WWTP. Thus, most of the conventional physicochemical and biological methods turn an IWW problem into a solid waste disposal problem (Sara Pérez-Elvira et al., 2017; Salehiziri et al., 2018). This is why the management of these solid wastes must be carried out from a sustainable approach in which some requirements are met in the efficient recycling of resources without having an environmental impact (Fytili \& Zabaniotou, 2008).

Similarly, as a result of the different physicochemical treatments in IWW, it is common to obtain inorganic sludge, compounds derived from metal cations, fluoride anions, cyanides, and phosphates, as well as 
synthetic organic compounds such as phenols, detergents, and oily emulsions (Lawrence K. Wang et al., 2005).

Although the easiest way to treat residual sludge is not to generate it in the first place, when there are no other means to reduce it, it must be treated seeking its reuse, recycling, revaluation, and ultimately its final disposal (Golomeova et al., 2013; Woodard \& Curran, 2006c). The most frequent technologies for the treatment of industrial solid waste, such as incineration or landfill, are subject to quite strict environmental quality requirements. Furthermore, the legislation on this application is increasingly stringent to avoid risks to human and environmental health due to potentially toxic elements in sewage sludge such as heavy metals, pathogens, and persistent organic pollutants (Sara Pérez-Elvira et al., 2017). However, trends in the field of sludge management correspond to technologies such as wet oxidation, pyrolysis, gasification, and combustion for later use as an energy source, have generated significant scientific interest (Elalami et al., 2019; Fytili \& Zabaniotou, 2008), among other alternatives such as synthesis of nanomaterials and formulation of building materials (Figure 9) (Anawar et al., 2019; Bernal et al., 2016; Bhatnagar et al., 2014; Bouargane et al., 2019; Guerra-Rodríguez et al., 2020; Martínez-Martínez et al., 2020; Norambuena-Contreras et al., 2018; Pérez-villarejo et al., 2015; Tsiligiannis \& Tsiliyannis, 2020; Tsiliyannis, 2017; Yoldi et al., 2019).

Within the future 3R scheme implementation, WWTP in the industry should convert to water-resourcerecovery from sludge and wastewater itself. Hence, in the future, sludge should be considered as feedstock for renewable energy and materials. For this purpose, emerging biological technologies such as dark fermentation, bioelectrochemical systems (BES), and others are currently under investigation (Elalami et al., 2019).

Advantages, disadvantages, and the potential to still exploit are presented in Table 4.For complete descriptions of every technology the followed cited investigation are recommended (Elalami et al., 2019; Fytili \& Zabaniotou, 2008; Golomeova et al., 2013; Mohanty et al., 2018; Safferman et al., 2017; Woodard \& Curran, 2006c). We also recommend the following investigations for minimization of sludge production in WWTP no cover in the present review (S. Pérez-Elvira et al., 2006; Sara Pérez-Elvira et al., 2017).

\section{General Future Perspective.}

As it was already discussed most of the WWTP implemented in the industry have the objective of removing pollutants from IWW, no recycling nor recovery (Ahmed et al., 2015). However, the cost of wastewater disposal and resources supply price, expected to increase even more pronounced shortly, will make the recovery of metals, nutrients and other materials that can be returned to the material cycles remain a challenge for the future (Burlakovs et al., 2018; Verhuelsdonk et al., 2021). Additionally, the nature of the recovered pollutant could become attractive and contributes to making an additional value to the existing industrial process. But still, significant efforts are required to enhance the process efficiencies and to develop newer separation options for recovery, recycling, and reuse.

As already seen, IWW treatment is a vast area covering several physicochemical and biological treatment methods which are commercially available and demonstrated that can recover resources and even produce energy. The appropriate application of these technologies can reduce pollution, mitigate global climate 
change, and create new industries and jobs since new applications can emerge for converting pollutants to useful materials rather than just removing and destroying them (Ranade \& Bhandari, 2014b; Safferman et al., 2017).

Emerging technologies, even they are promising for 3R schemes in IWW management, deal with several major difficulties, already mentioned, that may impair operations or prevent them from being commercially viable options, principally the energy consumption and general cost compared to the leading established technologies. For those established ones, they are mostly limited because they are not enough for the $3 R$ schemes with a low potential less to exploit, at least if they are not complemented with innovative, emerging, or adaptive use technologies. Nevertheless, it was shown that these established technologies still have space for improvement.

A proposed solution to all already mentioned problems is the 'Zero Liquid Discharge' or even more visionary the 'Zero Waste'. These correspond to any process or combination of processes in which there is no liquid effluent, waste, or discharge from industry (Ahirrao, 2014). This is attracting interest as a valuable water management solution for IWW by maximizing water recycling, minimizing wastewater volumes, and resource recovery (Muhammad Yaqub \& Lee, 2019). Nevertheless, Zero Waste is a myth since the current intensive energy use and usually expensive technology that has not yet been widely implemented, thus has urged research into finding alternatives or development of the emerging technologies. Even though, zero waste will stimulate innovation to develop and implement the 3R scheme in industry (Muhammad Yaqub \& Lee, 2019).

The cases of studies for the 3R scheme in different industries sectors, type of technology, or depending on the element interested in being valorized are already covered somewher eelse, the following cited papers are suggested (Goh et al., 2022; Hossain et al., 2020; Muhammad Yaqub \& Lee, 2019; Rezende Moreira et al., 2022; Verhuelsdonk et al., 2021; B. Yadav et al., 2020; G. Yadav et al., 2021).

\section{Conclusions}

Proper selection of wastewater treatment technology is the key to achieving recovery, recycling, and reuse to close the cycles of resources in the industry. However, even there is a wide range of possible technologies that correctly combined can achieve this, it was demonstrated that there is a lot of gaps and potential to exploit to make them economically and sustainably viable for a 3R scheme, even those mature massively implemented technologies in the industry. The goal is to implement zero waste systems, where even the solid waste generated from the water treatment is considered on large scale and much more competitive than traditional systems where just the disposal is considered. In general, to accomplish this, independent of the technology to be studied, the future investigation should be focused on: (1) The capacity in removing selected pollutants and decreasing impurities; (2) energy efficiency; (3) environmental safety; (4) economic viability; (5) hybrid processes, and (5) sustainability by reuse, recycle and recover.

\section{Declarations}


Funding: This work was funded by MINCIENCIAS - Ministry of Science, Technology, and Innovation of Colombia, and approved in the call 786-2017 for projects that aspire to obtain tax benefits for investment in CTel, project: 6765-786-59834.

Conflicts of interest/Competing interests: The authors have no relevant financial or non-financial interests to disclose.

Ethics approval: Not aplicable.

Consent to participate: Not aplicable.

Consent for publication: Not aplicable.

Availability of data and material: Not aplicable.

Code availability: Not aplicable.

Authors' contribution: All authors contributed to the study conception and design. Ricardo Mejía and Aymer Maturana had the idea for the article; the literature search was performed by Ricardo Mejia, Luis Arismendy and Carlos Cárdenas; The first draft of the manuscript was written by Ricardo Mejía. The manuscript was critically revised by Aymer Maturana, Diego Gómez and Christian Quintero. All authors read and approved the final manuscript.

\section{References}

1. Abdel-Karim A, Leaper S, Skuse C, Zaragoza G, Gryta M, Gorgojo P (2021) Membrane cleaning and pretreatments in membrane distillation - a review. Chem Eng J 422(March):129696. https://doi.org/10.1016/j.cej.2021.129696

2. Abdullah N, Yusof N, Lau WJ, Jaafar J, Ismail AF (2019) Recent trends of heavy metal removal from water/wastewater by membrane technologies. J Ind Eng Chem 76:17-38. https://doi.org/10.1016/j.jiec.2019.03.029

3. Accepta (1997) Cost-Effective Membrane Technologies for Minimising Wastes. In Environmental Technology Best Practice Programme (pp. 1-47)

4. Ahirrao S (2014) Zero Liquid Discharge Solutions. In Industrial Wastewater Treatment, Recycling and Reuse (pp. 489-520). Elsevier Ltd. https://doi.org/10.1016/B978-0-08-099968-5.00013-1

5. Ahmed M, Hasan CK, Rahman H, Hossain MA, Uddin SA (2015) Prospects of Using Wastewater as a Resource-Nutrient Recovery and Energy Generation. American Journal of Environmental Sciences Review 11(2). https://doi.org/10.3844/ajessp.2015.98

6. Ahmetovic E, Grossmann IE, Kravanja Z, Ibric N (2017) Water optimization in process industries. In Sustainable Utilization of Natural Resources (pp. 487-512). https://doi.org/10.1201/9781315153292

7. Al-Rashdi B, Somerfield C, Hilal N (2011) Heavy metals removal using adsorption and nanofiltration techniques. Sep Purif Rev 40(3):209-259. https://doi.org/10.1080/15422119.2011.558165 
8. Ali A, Quist-Jensen CA, Jørgensen MK, Siekierka A, Christensen ML, Bryjak M, Hélix-Nielsen C, Drioli E (2021) A review of membrane crystallization, forward osmosis and membrane capacitive deionization for liquid mining. Resources, Conservation and Recycling, 168(November 2020).

https://doi.org/10.1016/j.resconrec.2020.105273

9. Anawar HM, Strezov V, Hossain MZ (2019) Comparison of different nanoprocesses and industrial waste-based adsorbents such as red mud, steel slag, and fly ashes for treating wastewater nanomaterial contaminants. In: Mishra AK, Anawar HMD, Nadjib D (eds) Emerging and Nanomaterial Contaminants in Wastewater: Advanced Treatment Technologies, 1st edn. Elsevier Inc, pp 107-136. https://doi.org/10.1016/B978-0-12-814673-6.00005-X

10. Anderson J (2002) Prospects for international guidelines for water recycling. In A. Piet, Lens. Look, Hulshoff. Peter, Wilderer. Takashi (Ed.), Water recycling and resource recovery in industry: Analysism technologies and implementation (1st ed., pp. 161-179). IWA Publishing

11. Arabi S, Pellegrin ML, Aguinaldo J, Sadler ME, McCandless R, Sadreddini S, Wong J, Burbano MS, Koduri S, Abella K, Moskal J, Alimoradi S, Azimi Y, Dow A, Tootchi L, Kinser K, Kaushik V, Saldanha V (2020) Membrane processes. Water Environ Res 92(10):1447-1498. https://doi.org/10.1002/wer.1385

12. Asif MB, Hai FI, Jegatheesan V, Price WE, Nghiem LD, Yamamoto K (2018) Applications of membrane bioreactors in biotechnology processes. In: Bacile A, Charcosset $C$ (eds) Current Trends and Future Developments on (Bio-) Membranes: Membrane Processes in the Pharmaceutical and Biotechnological Field, 1st edn. Elsevier Inc, pp 223-257. https://doi.org/10.1016/B978-0-12-813606-5.00008-7

13. Baawain MS, Al-Mamun A, Omidvarborna H, Al-Sabti A, Choudri BS (2020) Public perceptions of reusing treated wastewater for urban and industrial applications: challenges and opportunities. Environ Dev Sustain 22(3):1859-1871. https://doi.org/10.1007/s10668-018-0266-0

14. Bastidas-Oyanedel J-R, Bonk F, Hedegaard Thomsen M, Ejbye Schimdt J (2019) The Future Perspectives of Dark Fermentation: Moving from Only Biohydrogen to Biochemicals. In: BastidasOyanedel J-R, Schmidt JE (eds) Biorefinery: Integrated Sustainable Processes for Biomass Conversion to Biomaterials, Biofuels and Fertilizers, 1st edn. Springer, pp 375-412

15. Bennamoun L (2012) An Overview on Application of Exergy and Energy for Determination of Solar Drying Efficiency. 2:184-194. https://doi.org/10.5923/j.ijee.20120205.01. 5

16. Bernal SA, Rodríguez ED, Kirchheim AP, Provis JL (2016) Management and valorisation of wastes through use in producing alkali-activated cement materials. J Chem Technol Biotechnol 91(9):23652388. https://doi.org/10.1002/jctb.4927

17. Bhandari VM, Ranade VV (2014) Advanced Physico-chemical Methods of Treatment for Industrial Wastewaters. Industrial Wastewater Treatment, Recycling and Reuse. Elsevier Ltd, pp 81-140. https://doi.org/10.1016/B978-0-08-099968-5.00002-7

18. Bhatnagar A, Kaczala F, Hogland W (2014) Valorization of solid waste products from olive oil industry as potential adsorbents for water pollution control - a review. Environ Sci Pollut Res 268-298. https://doi.org/10.1007/s11356-013-2135-6

19. Bhatt M, Chakinala AG, Joshi JB, Sharma A, Pant KK, Shah K, Sharma A (2021) Valorization of solid waste using advanced thermo-chemical process: A review. Journal of Environmental Chemical 
Engineering 9(4):105434. https://doi.org/10.1016/j.jece.2021.105434

20. Bhattacharya J, Dev S, Das B (2017) Design of Wastewater Bioremediation Plant and Systems. In Butterworth \& Heinemann (Eds.), Low Cost Wastewater Bioremediation Technology (1st ed., pp. 265313). Joe Hayton. https://doi.org/10.1016/b978-0-12-812510-6.00011-5

21. Bhuta H (2014) Advanced Treatment Technology and Strategy for Water and Wastewater Management. Industrial Wastewater Treatment, Recycling and Reuse. Elsevier Ltd, pp 193-213. https://doi.org/10.1016/B978-0-08-099968-5.00004-0

22. Biswas RK, Habib MA, Karmakar AK, Tanzin S (2016) Recovery of manganese and zinc from waste ZnC cell powder: Mutual separation of $\mathrm{Mn}(\mathrm{II})$ and $\mathrm{Zn}$ (II) from leach liquor by solvent extraction technique. Waste Manag 51:149-156. https://doi.org/10.1016/j.wasman.2015.09.041

23. Blair J, Mataraarachchi S (2021) A Review of Landfills, Waste and the Nearly Forgotten Nexus with Climate Change. Environments 8(73):1-25

24. Bottrel C, Amorim C, Leão MD, Costa P, Lacerda A (2014) Degradation of ethylenethiourea pesticide metabolite from water by photocatalytic processes. Journal of Environmental Science and Health - Part B Pesticides, Food Contaminants, and Agricultural Wastes 49(4):263-270. https://doi.org/10.1080/03601234.2014.868280

25. Bouargane B, Marrouche A, El Issiouy S, Biyoune MG, Mabrouk A, Atbir A, Bachar A, Bellajrou R, Boukbir L, Bakiz B (2019) Recovery of $\mathrm{Ca}(\mathrm{OH}) 2, \mathrm{CaCO} 3$, and Na2SO4 from Moroccan phosphogypsum waste. J Mater Cycles Waste Manage 21(6):1563-1571. https://doi.org/10.1007/s10163-019-00910-9

26. Buccolieri A, Serra A, Maruccio G, Monteduro AG, Padmanabhan SK, Licciulli A, Bonfrate V, Salvatore L, Manno D, Calcagnile L, Giancane G (2017) Synthesis and Characterization of Mixed Iron-Manganese Oxide Nanoparticles and Their Application for Efficient Nickel Ion Removal from Aqueous Samples. Journal of Analytical Methods in Chemistry, 2017, 1-9. https://doi.org/10.1155/2017/9476065

27. Burlakovs J, Jani Y, Kriipsalu M, Vincevica-Gaile Z, Kaczala F, Celma G, Ozola R, Rozina L, Rudovica V, Hogland M, Viksna A, Pehme KM, Hogland W, Klavins M (2018) On the way to 'zero waste' management: Recovery potential of elements, including rare earth elements, from fine fraction of waste. J Clean Prod 186:81-90. https://doi.org/10.1016/j.jclepro.2018.03.102

28. Cecconet D, Sabba F, Devecseri M, Callegari A, Capodaglio AG (2020) In situ groundwater remediation with bioelectrochemical systems: A critical review and future perspectives. Environ Int 137(January). https://doi.org/10.1016/j.envint.2020.105550

29. Chen WS, Liao CT, Lin KY (2017) Recovery Zinc and Manganese from Spent Battery Powder by Hydrometallurgical Route. Energy Procedia, 107(September 2016), 167-174. https://doi.org/10.1016/j.egypro.2016.12.162

30. Colón J, Alarcón M, Healy MG, Namli A, Sanin FD, Tayà C, Ponsà S (2017) Producing sludge for agricultural applications. In J. Lema \& S. Suarez (Eds.), Innovative Wastewater Treatment \& Resource Recovery Technologies: Impacts on Energy, Economy and Environment (1st ed., pp. 296-322). IWA Publishing. https://doi.org/10.2166/9781780407876_0296

31. Costa CZ, Sousa-Aguiar EF, Couto MAPG, de Filho JFS C (2020) Hydrothermal treatment of vegetable oils and fats aiming at yielding hydrocarbons: A review. Catalysts 10(8). 
https://doi.org/10.3390/catal10080843

32. Crini G, Lichtfouse E (2019) Advantages and disadvantages of techniques used for wastewater treatment. Environ Chem Lett 17(1):145-155. https://doi.org/10.1007/s10311-018-0785-9

33. Cui ZF, Jiang Y, Field RW (2010) Fundamentals of Pressure-Driven Membrane Separation Processes. In Z.. Cui \& H. S. Muralidhara (Eds.), Membrane Technology (First Edit, pp. 1-18). Butterworth-Heinemann. https://doi.org/10.1016/B978-1-85617-632-3.00001-X

34. Das S, Bhat AP, Gogate PR (2021) Degradation of dyes using hydrodynamic cavitation: Process overview and cost estimation. Journal of Water Process Engineering 42(January):102126. https://doi.org/10.1016/j.jwpe.2021.102126

35. Dawn SS, Vishwakarma V (2021) Chemosphere Recovery and recycle of wastewater contaminated with heavy metals using adsorbents incorporated from waste resources and nanomaterials-A review. Chemosphere 273:129677. https://doi.org/10.1016/j.chemosphere.2021.129677

36. Dayarathne HNP, Angove MJ, Aryal R, Abuel-Naga H, Mainali B (2021) Removal of natural organic matter from source water: Review on coagulants, dual coagulation, alternative coagulants, and mechanisms. Journal of Water Process Engineering, 40(December 2020), 101820. https://doi.org/10.1016/j.jwpe.2020.101820

37. De Luna MDG, Rance DPM, Bellotindos LM, Lu MC (2017) Removal of sulfate by fluidized bed crystallization process. Journal of Environmental Chemical Engineering 5(3):2431-2439. https://doi.org/10.1016/j.jece.2017.04.052

38. Devda V, Chaudhary K, Varjani S, Pathak B, Patel AK, Singhania RR, Taherzadeh MJ, Ngo HH, Wong JWC, Guo W, Chaturvedi P (2021) Recovery of resources from industrial wastewater employing electrochemical technologies: status, advancements and perspectives. Bioengineered 12(1):46974718. https://doi.org/10.1080/21655979.2021.1946631

39. Drewes JE, Horstmeyer N, Michel P, Khan S (2017) Producing high-quality recycled water. In: Lema J, Suarez S (eds) Innovative Wastewater Treatment \& Resource Recovery Technologies: Impacts on Energy, Economy and Environment, 1st edn. IWA Publishing London, pp 285-295. https://doi.org/10.2166/9781780407876_0285

40. Elalami D, Carrere H, Monlau F, Abdelouahdi K, Oukarroum A, Barakat A (2019) Pretreatment and codigestion of wastewater sludge for biogas production: Recent research advances and trends. Renew Sustain Energy Rev 114(February):109287. https://doi.org/10.1016/j.rser.2019.109287

41. Elliott JAK, Ball AS (2021) Selection of industrial trade waste resource recovery technologies-A systematic review. Resources 10(4). https://doi.org/10.3390/resources10040029

42. Eykamp W (1995) Microfiltration and ultrafiltration. In R. D. Noble \& S. A. Stern (Eds.), Membrane Science and Technology (1st ed., Vol. 2, pp. 1-43). https://doi.org/10.1016/S0927-5193(06)80003-3

43. Ezugbe EO, Rathilal S (2020) Membrane technologies in wastewater treatment: A review. Membranes 10(5). https://doi.org/10.3390/membranes10050089

44. Farhat NM, Christodoulou C, Placotas P, Blankert B, Sallangos O, Vrouwenvelder JS (2020) Cartridge filter selection and replacement: Optimization of produced water quantity, quality, and cost. Desalination, 473(July 2019), 114172. https://doi.org/10.1016/j.desal.2019.114172 
45. Fernández-alba AR (2006) Tratamientos avanzados de aguas residuales industriales (J. De la Sota (ed.); 1 st). citme

46. Fernández-Torres MJ, Randall DG, Melamu R, von Blottnitz H (2012) A comparative life cycle assessment of eutectic freeze crystallisation and evaporative crystallisation for the treatment of saline wastewater. Desalination 306:17-23. https://doi.org/10.1016/j.desal.2012.08.022

47. Folaranmi G, Bechelany M, Sistat P, Cretin M, Zaviska F (2020) Towards electrochemical water desalination techniques: A review on capacitive deionization, membrane capacitive deionization and flow capacitive deionization. Membranes 10(5). https://doi.org/10.3390/membranes10050096

48. Fytili D, Zabaniotou A (2008) Utilization of sewage sludge in EU application of old and new methods-A review. Renew Sustain Energy Rev 12(1):116-140. https://doi.org/10.1016/j.rser.2006.05.014

49. Galinha CF, Sanches S, Crespo JG (2018) Membrane bioreactors. In P. Luis (Ed.), Fundamental Modeling of Membrane Systems: Membrane and Process Performance (1st ed., pp. 209-249). Susan Dennis. https://doi.org/10.1016/B978-0-12-813483-2.00006-X

50. Genc S, Akhisaroglu M, Kuralay F, Genc K (2002) Erythropoietin restores glutathione peroxidase activity in 1-methyl-4-phenyl-1,2,5,6-tetrahydropyridine-induced neurotoxicity in C57BL mice and stimulates murine astroglial glutathione peroxidase production in vitro. Neurosci Lett 321(1-2):73-76. https://doi.org/10.1016/S0304-3940(02)00041-1

51. Ghorbani Y, Franzidis JP, Petersen J (2016) Heap leaching technology - Current State, innovations, and future directions: A review. Miner Process Extr Metall Rev 37(2):73-119.

https://doi.org/10.1080/08827508.2015.1115990

52. Giwa A, Yusuf A, Balogun HA, Sambudi NS, Bilad MR, Adeyemi I, Chakraborty S, Curcio S (2021) Recent advances in advanced oxidation processes for removal of contaminants from water: A comprehensive review. Process Saf Environ Prot 146:220-256. https://doi.org/10.1016/j.psep.2020.08.015

53. Goh PS, Wong KC, Ismail AF (2022) Membrane technology: A versatile tool for saline wastewater treatment and resource recovery. Desalination, 521(July 2021), 115377. https://doi.org/10.1016/j.desal.2021.115377

54. Goli VSNS, Singh DN, Baser T (2021) A critical review on thermal treatment technologies of combustible fractions from mechanical biological treatment plants. Journal of Environmental Chemical Engineering 9(4):105643. https://doi.org/10.1016/j.jece.2021.105643

55. Golomeova S, Srebrenkoska V, Krstevas S, Spasovas S (2013) Solid Waste Treatment Technologies. Scientific Proceedings X International Congress "Machines, Technologies, Material," 2, 63-65. http://www.psemr.vojvodina.gov.rs

56. Green D, Perry R (2015) Perry's Chemical Engineers' Handbook (8th Editio, Vol. 3, Issue 2). McGraw-Hill. http://repositorio.unan.edu.ni/2986/1/5624.pdf

57. Guerra-Rodríguez S, Oulego P, Rodríguez E, Singh DN, Rodríguez-Chueca J (2020) Towards the implementation of circular economy in the wastewater sector: Challenges and opportunities. Water (Switzerland) 12(5). https://doi.org/10.3390/w12051431

58. Guo J, Xu Y (2011) Review of Enzymatic Sludge Hydrolysis. Journal of Bioremediation and Biodegradation 02(05). https://doi.org/10.4172/2155-6199.1000130 
59. Gupta VK, Tyagi I, Sadegh H, Ghoshekand RS-, Makhlouf ASH, Maazinejad B (2015) Nanoparticles as Adsorbent; A Positive Approach for Removal of Noxious Metal Ions: A Review. Science, Technology and Development 34(3):195-214. https://doi.org/10.3923/std.2015.195.214

60. Hamedi H, Ehteshami M, Mirbagheri SA, Rasouli SA, Zendehboudi S (2019) Current Status and Future Prospects of Membrane Bioreactors (MBRs) and Fouling Phenomena: A Systematic Review. Can J Chem Eng 97(1):32-58. https://doi.org/10.1002/cjce.23345

61. Handojo L, Wardani AK, Regina D, Bella C, Kresnowati MTAP, Wenten IG (2019) Electro-membrane processes for organic acid recovery. RSC Adv 9:7854-7869. https://doi.org/10.1039/C8RA09227C

62. Hassan SR, Zwain HM, Dahlan I (2013) Development of Anaerobic Reactor for Industrial Wastewater Treatment: An Overview, Present Stage and Future Prospects. Journal of Advanced Scientific Research 4(1):7-12

63. Helmreich B, Metzger S (2017) Post-treatment for micropollutants removal. In J. Lema \& S. Suarez (Eds.), Innovative Wastewater Treatment \& Resource Recovery Technologies: Impacts on Energy, Economy and Environment (1st ed., pp. 214-232). IWA Publishing.

https://doi.org/10.2166/9781780407876_0214

64. Hossain N, Bhuiyan MA, Pramanik BK, Nizamuddin S, Griffin G (2020) Waste materials for wastewater treatment and waste adsorbents for biofuel and cement supplement applications: A critical review. Journal of Cleaner Production, 255. https://doi.org/10.1016/j.jclepro.2020.120261

65. Innocenzi V, Veglio F (2012) Separation of manganese, zinc and nickel from leaching solution of nickelmetal hydride spent batteries by solvent extraction. Hydrometallurgy 129-130:50-58.

https://doi.org/10.1016/j.hydromet.2012.08.003

66. Iorhemen OT, Hamza RA, Tay JH (2016) Membrane bioreactor (Mbr) technology for wastewater treatment and reclamation: Membrane fouling. Membranes 6(2):13-16.

https://doi.org/10.3390/membranes6020033

67. Ives KJ (1970) Review Paper - Rapid Filtration. Rapid Filtration 4(1):201-223

68. Jana S, Gnanakan K, Jana BB (2018) Multiple reuse of wastewater: Economic perspectives. In Wastewater Management Through Aquaculture (pp. 255-267). https://doi.org/10.1007/978-981-107248-2_13

69. Janajreh I, Adeyemi I, Raza SS, Ghenai C (2021) A review of recent developments and future prospects in gasification systems and their modeling. Renewable and Sustainable Energy Reviews, 138(October 2020), 110505. https://doi.org/10.1016/j.rser.2020.110505

70. Jaroszek H, Dydo P (2016a) lon-exchange membranes in chemical synthesis-a review. Open Chemistry 14(1):1-19. https://doi.org/10.1515/chem-2016-0002

71. Jaroszek H, Dydo P (2016b) lon-exchange membranes in chemical synthesis-a review. Open Chemistry 14(1):1-19. https://doi.org/10.1515/chem-2016-0002

72. Johnson DB (2018) The evolution, current status, and future prospects of using biotechnologies in the mineral extraction and metal recovery sectors. Minerals 8(8):1-14.

https://doi.org/10.3390/min8080343 
73. Judd SJ (2002) Industrial experience of water reuse by membrane technology. In A. Piet, Lens. Look, Hulshoff. Peter, Wilderer. Takashi (Ed.), Water recycling and resource recovery in industry: Analysism technologies and implementation (1st ed., pp. 472-487). IWA Publishing

74. Kamali M, Sweygers N, Al-Salem S, Appels L, Aminabhavi TM, Dewil R (2022) Biochar for soil applications-sustainability aspects, challenges and future prospects. Chemical Engineering Journal, 428(March 2021), 131189. https://doi.org/10.1016/j.cej.2021.131189

75. Kamarulzaman A, Hasanuzzaman M, Rahim NA (2021) Global advancement of solar drying technologies and its future prospects: A review. Solar Energy, 221(August 2020), 559-582. https://doi.org/10.1016/j.solener.2021.04.056

76. Kinidi L, Ai I, Tan W, Binti N, Wahab A, Fikri K, Tamrin B, Hipolito CN, Salleh SF (2018) Recent Development in Ammonia Stripping Process for Industrial Wastewater Treatment. International Journal of Chemical Engineering, 2018, 14

77. Knez Ž, Pantić M, Cör D, Novak Z, Knez Hrnčič M (2019) Are supercritical fluids solvents for the future? Chemical Engineering and Processing - Process Intensification, 141(December 2018). https://doi.org/10.1016/j.cep.2019.107532

78. Kyzas GZ (2018) Flotation in Water and Wastewater Treatment. Processes 6(116):1-16. https://doi.org/10.3390/pr6080116

79. Levine AD, Asano T (2002) Water reclamation, recycling, and reuse in industry. In: Lens T, Hulshoff $P$, Wilderer $\mathrm{L}$, Asano $\mathrm{P}$ (eds) Water recycling and resource recovery in industry: Analysism technologies and implementation, 1st edn. IWA Publishing, pp 29-50

80. Ljunggren $M$ (2006) Micro screening in wastewater treatment - an overview. Vatten 62:171-177

81. Lu H, Wang J, Wang T, Wang N, Bao Y, Hao H (2017) Crystallization techniques in wastewater treatment: An overview of applications. Chemosphere 173:474-484. https://doi.org/10.1016/j.chemosphere.2017.01.070

82. Luz E, Bashan Y (2004) Recent advances in removing phosphorus from wastewater and its future use as fertilizer (1997 - 2003). Water Res 38:4222-4246. https://doi.org/10.1016/j.watres.2004.07.014

83. Ma P, Rosen C (2021) Land application of sewage sludge incinerator ash for phosphorus recovery: A review. Chemosphere 274:129609. https://doi.org/10.1016/j.chemosphere.2021.129609

84. Mahto A, Aruchamy K, Meena R, Kamali M (2021) Forward osmosis for industrial effluents treatment sustainability considerations. Separation and Purification Technologies, 254(August 2020). https://doi.org/10.1016/j.seppur.2020.117568

85. Mai DT, Kunacheva C, Stuckey DC (2018) A review of posttreatment technologies for anaerobic effluents for discharge and recycling of wastewater. Crit Rev Environ Sci Technol 48(2):167-209. https://doi.org/10.1080/10643389.2018.1443667

86. Martínez-Martínez S, Pérez-Villarejo L, Eliche-Quesada D, Sánchez-Soto PJ, Christogerou A, Kanellopoulou DG, Angelopoulos GN (2020) New waste-based clinkers for the preparation of lowenergy cements. A step forward toward circular economy. Int J Appl Ceram Technol 17(1):12-21. https://doi.org/10.1111/ijac.13390 
87. Maurer M, Muncke J, Larsen T (2002) Technologies for nitrogen recovery and reuse. In A. Piet, Lens. Look, Hulshoff. Peter, Wilderer. Takashi (Ed.), Water recycling and resource recovery in industry: Analysism technologies and implementation (1st ed., Issue 3, pp. 491-510). IWA Publishing

88. Mejía Marchena R, Maturana Córdoba A, Gomez Cerón D, Monroy Q, Montes CArismendy, Perez C (2020) C. Reuse of manganese sulfate as raw material by recovery from pesticide's wastewater using nanofiltration and electro-electrodialysis: process simulation and analysis from actual data. Water Science and Technology, wst2020179, 1-16. https://doi.org/10.2166/wst.2020.179

89. Mels AR, Teerikangas E (2002) Physico - chemical wastewater treatment. In A. Piet, Lens. Look, Hulshoff. Peter, Wilderer. Takashi (Ed.), Water recycling and resource recovery in industry: Analysism technologies and implementation (1sr ed., pp. 433-451). IWA Publishing

90. Mocellin J, Mercier G, Morel JL, Charbonnier P, Blais JF, Simonnot MO (2017) Recovery of zinc and manganese from pyrometallurgy sludge by hydrometallurgical processing. J Clean Prod 168:311-321. https://doi.org/10.1016/j.jclepro.2017.09.003

91. Mohanty S, Ghosh S, Bal B, Das AP (2018) A review of biotechnology processes applied for manganese recovery from wastes. Rev Environ Sci Biotechnol 17(4):791-811. https://doi.org/10.1007/s11157-0189482-1

92. Mohapatra DP, Kirpalani DM (2019) Advancement in treatment of wastewater: Fate of emerging contaminants. Can J Chem Eng 97(10):2621-2631. https://doi.org/10.1002/cjce.23533

93. Mohsin ASM, Maruf MNI, Sayem AHM, Mojumdar MRR, Farhad S, H. M (2011) Prospect \& Future of Solar Dryer: Perspective Bangladesh. International Journal of Engineering and Technology 3(2):165170. https://doi.org/10.7763/ijet.2011.v3.217

94. Mohtashami R, Shang JQ (2019) Electroflotation for Treatment of Industrial Wastewaters: A Focused Review. Environmental Processes 6(2):325-353. https://doi.org/10.1007/s40710-019-00348-z

95. Morales-arrieta S, Okoye PU, Ang R (2021) Recycling industrial wastewater for improved carbohydraterich biomass production in a semi-continuous photobioreactor: Effect of hydraulic retention time. $\mathrm{J}$ Environ Manage 284(January):2-10. https://doi.org/10.1016/j.jenvman.2021.112065

96. Moran S (2018) Dirty water unit operation design. In Elseiver (Ed.), An Applied Guide to Water and Effluent Treatment Plant Design (1st ed., pp. 171-202). Joe Hayton. https://doi.org/10.1016/b978-0-12811309-7.00014-x

97. Mpofu AB, Oyekola OO, Welz PJ (2021) Anaerobic treatment of tannery wastewater in the context of a circular bioeconomy for developing countries. J Clean Prod 296:126490. https://doi.org/10.1016/j.jclepro.2021.126490

98. Muddemann T, Haupt D, Sievers M, Kunz U (2019) Electrochemical Reactors for Wastewater Treatment. ChemBioEng Reviews 6(5):142-156. https://doi.org/10.1002/cben.201900021

99. Muhammad Yaqub, Lee W (2019) Zero-liquid discharge (ZLD) technology for resource recovery from wastewater: A review. Sci Total Environ 681:551-563. https://doi.org/10.1016/j.scitotenv.2019.05.062

100. Naqvi SR, Tariq R, Shahbaz M, Naqvi M, Aslam M, Khan Z, Mackey H, Mckay G, Al-Ansari T (2021) Recent developments on sewage sludge pyrolysis and its kinetics: Resources recovery, 
thermogravimetric platforms, and innovative prospects. Comput Chem Eng 150:107325. https://doi.org/10.1016/j.compchemeng.2021.107325

101. Nidheesh PV, Ravindran V, Gopinath A, Kumar MS (2021) Emerging technologies for mixed industrial wastewater treatment in developing countries: An overview. Environmental Quality Management, January 2020,1-21. https://doi.org/10.1002/tqem.21762

102. Norambuena-Contreras J, Quilodran J, Gonzalez-Torre I, Chavez M, Borinaga-Treviño R (2018) Electrical and thermal characterisation of cement-based mortars containing recycled metallic waste. J Clean Prod 190:737-751. https://doi.org/10.1016/j.jclepro.2018.04.176

103. Nowak M, Jaroszek H, Turkowska M (2014) Conversion of waste sodium sulfate with bipolar membrane electrodialysis. Membranes and Membrane Processes in Environmental Protection 119(June):337-349

104. Nunes SP, Culfaz-Emecen PZ, Ramon GZ, Visser T, Koops GH, Jin W, Ulbricht M (2020) Thinking the future of membranes: Perspectives for advanced and new membrane materials and manufacturing processes. Journal of Membrane Science, 598(October 2019), 117761.

https://doi.org/10.1016/j.memsci.2019.117761

105. Ødegaard H, Liao Z, Hansen AT (2002) Coarse media filtration - an alternative to settling in wastewater treatment. Water Sci Technol 47(12):81-88

106. Oren $Y(2008)$ Capacitive deionization (CDI) for desalination and water treatment - past, present and future (a review). Desalination 228(1-3):10-29. https://doi.org/10.1016/j.desal.2007.08.005

107. Paidar M, Fateev V, Bouzek K (2016) Membrane electrolysis-History, current status and perspective. Electrochim Acta 209:737-756. https://doi.org/10.1016/j.electacta.2016.05.209

108. Papatzani S (2019) A review on the valorization of marble dust/solids or slurry: Classification, current trends and potentials. Indian Concrete Journal 93(9):36-54

109. Patil DS, Chavan SM, Oubagaranadin JUK (2016) A review of technologies for manganese removal from wastewaters. Journal of Environmental Chemical Engineering 4(1):468-487. https://doi.org/10.1016/j.jece.2015.11.028

110. Peng W, Chang L, Li P, Han G, Huang Y, Cao Y (2019) An overview on the surfactants used in ion flotation. Journal of Molecular Liquids, 286. https://doi.org/10.1016/j.molliq.2019.110955

111. Perera MK, Englehardt JD, Dvorak AC (2019) Technologies for Recovering Nutrients from Wastewater: A Critical Review. Environ Eng Sci 36(5):511-529. https://doi.org/10.1089/ees.2018.0436

112. Pérez-Elvira S, Diez N, Fdz-Polanco F (2006) Sludge minimisation technologies. Rev Environ Sci Biotechnol 5(4):375-398. https://doi.org/10.1007/s11157-005-5728-9

113. Pérez-Elvira S, Fdz-Polanco F, Tomei MC, Bertanza G, Braguglia CM, Gianico A, Carrére H (2017) Reducing the impact of sludge. In J. Lema \& S. Suarez (Eds.), Innovative Wastewater Treatment \& Resource Recovery Technologies: Impacts on Energy, Economy and Environment (1 st, pp. 255-282). IWA Publishing. https://doi.org/10.2166/9781780407876_0255

114. Pérez-villarejo L, Martínez-martínez S, Carrasco-hurtado B, Eliche-quesada D (2015) Applied Clay Science Valorization and inertization of galvanic sludge waste in clay bricks. Appl Clay Sci 105106:89-99. https://doi.org/10.1016/j.clay.2014.12.022 
115. Perez JPH, Folens K, Leus K, Vanhaecke F, Van Der Voort P, Du Laing G (2019) Progress in hydrometallurgical technologies to recover critical raw materials and precious metals from lowconcentrated streams. Resources, Conservation and Recycling, 142(December 2018), 177-188. https://doi.org/10.1016/j.resconrec.2018.11.029

116. Piadeh F, Moghaddam MRA, Mardan S (2014) Present situation of wastewater treatment in the Iranian industrial estates: Recycle and reuse as a solution for achieving goals of eco-industrial parks. Resour Conserv Recycl 92:172-178. https://doi.org/10.1016/j.resconrec.2014.06.004

117. Pisarska B, Jaroszek H, Mikołajczak W, Nowak M, Cichy B, Stopa H, Markowicz P (2017) Application of electro-electrodialysis for processing of sodium sulphate waste solutions containing organic compounds: Preliminary study. J Clean Prod 142:3741-3747.

https://doi.org/10.1016/j.jclepro.2016.10.092

118. Pisarska B, Mikołajczak W, Jaroszek H, Nowak M, Dylewski R, Cichy B (2017) Processing of sodium sulphate solutions using the EED method: From a batch toward a continuous process. Polish Journal of Chemical Technology 19(1):54-58. https://doi.org/10.1515/pjct-2017-0008

119. Ponsa S, Bolzonella D, Colon J, Deshusses MA, Fonts I, Gil-Lalaguna N, Komilis D, Lyberatos G, PérezElvira SI, Sánchez JL (2017) Recovering energy from sludge. In J. Lema \& S. Suarez (Eds.), Innovative Wastewater Treatment \& Resource Recovery Technologies: Impacts on Energy, Economy and Environment (1st ed., pp. 325-354). IWA Publishing. https://doi.org/10.2166/9781780407876_0325

120. Porada S, Zhao R, Van Der Wal A, Presser V, Biesheuvel PM (2013) Review on the science and technology of water desalination by capacitive deionization. Prog Mater Sci 58(8):1388-1442. https://doi.org/10.1016/j.pmatsci.2013.03.005

121. Poyatos JM, Muñio MM, Almecija MC, Torres JC, Hontoria E, Osorio F (2010) Advanced oxidation processes for wastewater treatment: State of the art. Water Air Soil Pollut 205(1-4):187-204. https://doi.org/10.1007/s11270-009-0065-1

122. Ramírez Calderón OA, Abdeldayem OM, Pugazhendhi A, Rene ER (2020) Current Updates and Perspectives of Biosorption Technology: an Alternative for the Removal of Heavy Metals from Wastewater. Current Pollution Reports 6(1):8-27. https://doi.org/10.1007/s40726-020-00135-7

123. Ranade VV, Bhandari VM (2014a) Industrial Wastewater Treatment, Recycling, and Reuse-Past, Present and Future. Industrial Wastewater Treatment, Recycling and Reuse. Elsevier Ltd, pp 521-535. https://doi.org/10.1016/B978-0-08-099968-5.00014-3

124. Ranade VV, Bhandari VM (2014b) Industrial Wastewater Treatment, Recycling, and Reuse: An Overview. Industrial Wastewater Treatment, Recycling and Reuse. Elsevier Ltd, pp 1-80. https://doi.org/10.1016/B978-0-08-099968-5.00001-5

125. Rezende Moreira V, Abner Rocha Lebron Y, Amaral CS, M (2022) Enhancing industries exploitation: Integrated and hybrid membrane separation processes applied to industrial effluents beyond the treatment for disposal. Chem Eng J 430(P3):133006. https://doi.org/10.1016/j.cej.2021.133006

126. Roy A, Bhattacharya J (2014) Nanotechnology in Industrial Wastewater Treatment. In Water Intelligence Online. IWA Publishing 13. 1st edn.https://doi.org/10.2166/9781780406886 
127. Safferman SI, Smith JS, Dong Y, Saffron CM, Wallace JM, Binkley D, Thomas MR, Miller SA, Bissel E, Booth J, Lenz J (2017) Resources from Wastes: Benefits and Complexity. J Environ Eng 143(11):03117005. https://doi.org/10.1061/(asce)ee.1943-7870.0001259

128. Saharan VK, Pinjari DV, Gogate PR, Pandit AB (2014) Advanced Oxidation Technologies for Wastewater Treatment: An Overview. Industrial Wastewater Treatment, Recycling and Reuse. Elsevier Ltd, pp 141191. https://doi.org/10.1016/B978-0-08-099968-5.00003-9

129. Salehiziri M, Rad A, Novak JT (2018) Disruption of cell to cell communication in the aeration unit of a cannibal process: Sludge reduction efficiency and related mechanisms. Biochem Eng J 137:326-333. https://doi.org/10.1016/j.bej.2018.06.015

130. Sayilgan E, Kukrer T, Yigit NO, Civelekoglu G, Kitis M (2010) Acidic leaching and precipitation of zinc and manganese from spent battery powders using various reductants. J Hazard Mater 173(1-3):137143. https://doi.org/10.1016/j.jhazmat.2009.08.063

131. Shrestha R, Ban S, Devkota S, Sharma S, Joshi R, Tiwari AP, Kim HY, Joshi MK (2021) Technological trends in heavy metals removal from industrial wastewater: A review. Journal of Environmental Chemical Engineering 9(4):105688. https://doi.org/10.1016/j.jece.2021.105688

132. Shyam S, Arun J, Gopinath KP, Ribhu G, Ashish M, Ajay S (2022) Biomass as source for hydrochar and biochar production to recover phosphates from wastewater: A review on challenges, commercialization, and future perspectives. Chemosphere 286(P1):131490. https://doi.org/10.1016/j.chemosphere.2021.131490

133. Simon RG, Stöckl M, Becker D, Steinkamp AD, Abt C, Jungfer C, Weidlich C, Track T, Mangold KM (2018) Current to Clean Water - Electrochemical Solutions for Groundwater, Water, and Wastewater Treatment. Chem-Ing-Tech 90(11):1832-1854. https://doi.org/10.1002/cite.201800081

134. Singh R, Samal K, Dash RR, Bhunia P (2019) Vermifiltration as a sustainable natural treatment technology for the treatment and reuse of wastewater: A review. Journal of Environmental Management, 247(December 2018), 140-151. https://doi.org/10.1016/j.jenvman.2019.06.075

135. Smol M, Adam C, Preisner M (2020) Circular economy model framework in the European water and wastewater sector. J Mater Cycles Waste Manage 22(3):682-697. https://doi.org/10.1007/s10163019-00960-z

136. Srinivasan A, Chowdhury P, Viraraghavan T (2008) Air Stripping in Industrial Waste Water Treatment. In: Vigneswaran S (ed) Water and wastewater treatment technologies, 1st edn. EOLSS publisher, pp 88110

137. Stanbury PF, Whitaker A, Hall SJ (2016) Effluent treatment. In Butterworth \& Heinemann (Eds.), Principles of Fermentation Technology (3rd ed., pp. 687-723). Joe Hayton. https://doi.org/https://doi.org/10.1016/B978-0-08-099953-1.00011-9

138. Stessel R (1996) Recycling and Resource Recovery Engineering. In U. Förstner, J. R. Murohy, \& W. H. Rulkens (Eds.), Military Engineer (1st editio, Issue 712). Springer

139. Strehler JL, Gauff R, Miner E, Turgeon J, Valero D (2018) Sustainability and energy management for water resource recovery facilities. In ASCE Manuals and Reports on Engineering Practice (1st ed., Vols. 2018-Janua, Issue 137). WEF, ASCE/EWRI. https://doi.org/10.1061/9780784481233 
140. Tahreen A, Jami MS, Ali F (2020) Role of electrocoagulation in wastewater treatment: A developmental review. Journal of Water Process Engineering 37(April):101440.

https://doi.org/10.1016/j.jwpe.2020.101440

141. Tambone F, Scaglia B, Scotti S, Adani F (2011) Bioresource Technology Effects of biodrying process on municipal solid waste properties. Bioresour Technol 102(16):7443-7450.

https://doi.org/10.1016/j.biortech.2011.05.010

142. Thuptimdang P, Siripattanakul-Ratpukdi S, Ratpukdi T, Youngwilai A, Khan E (2020) Biofiltration for treatment of recent emerging contaminants in water: Current and future perspectives. Water Environ Res 0-2. https://doi.org/10.1002/wer.1493

143. Tong X, Cui Y, Wang YH, Bai Y, Yu T, Zhao XH, Ikuno N, Luo H, jia, Hu HY, Wu YH (2020) Fouling properties of reverse osmosis membranes along the feed channel in an industrial-scale system for wastewater reclamation. Sci Total Environ 713:136673.

https://doi.org/10.1016/j.scitotenv.2020.136673

144. Tran HT, Lin C, Bui XT, Ngo HH, Cheruiyot NK, Hoang HG, Vu CT (2021) Aerobic composting remediation of petroleum hydrocarbon-contaminated soil. Current and future perspectives. Sci Total Environ 753:142250. https://doi.org/10.1016/j.scitotenv.2020.142250

145. Tsiligiannis A, Tsiliyannis $C$ (2020) Oil refinery sludge and renewable fuel blends as energy sources for the cement industry. Renewable Energy 157:55-70. https://doi.org/10.1016/j.renene.2020.03.129

146. Tsiliyannis CA (2017) Industrial Wastes and By-products as Alternative Fuels in Cement Plants Evaluation of an Industrial Symbiosis Option. 22. https://doi.org/10.1111/jiec.12644. 5

147. Urbina-suarez NA, Machuca-martínez F, Barajas-solano AF (2021) Advanced oxidation processes and biotechnological alternatives for the treatment of tannery wastewater. Molecules 26(11):1-25. https://doi.org/10.3390/molecules 26113222

148. Van de Worp JJM (2002) Sustainable water management in industry. In: Lens P, Hulshoff L, Wilderer P, Asano T (eds) Water recycling and resource recovery in industry: Analysism technologies and implementation, 1st edn. IWA Publishing, pp 3-26

149. Van der Bruggen B (2018) Ion-exchange membrane systems-Electrodialysis and other electromembrane processes. In: Luis P (ed) Fundamental Modeling of Membrane Systems: Membrane and Process Performance, 1st edn. Elsevier Inc, pp 251-300. https://doi.org/10.1016/B978-0-12-813483-2.00007-1

150. Varjani S, Joshi R, Srivastava VK, Ngo HH, Guo W (2020) Treatment of wastewater from petroleum industry: current practices and perspectives. Environ Sci Pollut Res 27(22):27172-27180. https://doi.org/10.1007/s11356-019-04725-x

151. Varjani S, Shah AV, Vyas S, Srivastava VK (2021) Processes and prospects on valorizing solid waste for the production of valuable products employing bio-routes: A systematic review. Chemosphere 282(May):130954. https://doi.org/10.1016/j.chemosphere.2021.130954

152. Veit HM, Bernardes AM (2015) Electronic waste: Recycling techniques. In H. M. Veit \& A. Moura Bernardes (Eds.), Electronic Waste: Recycling Techniques (1st ed., pp. 39-59). Springer. https://doi.org/10.1007/978-3-319-15714-6 
153. Verhuelsdonk M, Glas K, Parlar H (2021) Economic evaluation of the reuse of brewery wastewater. Journal of Environmental Management, 281(December 2020), 111804. https://doi.org/10.1016/j.jenvman.2020.111804

154. Vogelpohl A (2002) Advanced oxidation technologies for industrial water reuse. In A. Piet, Lens. Look, Hulshoff. Peter, Wilderer. Takashi (Ed.), Water recycling and resource recovery in industry: Analysism technologies and implementation (1st ed., pp. 453-469). IWA Publishing

155. Wang G, Dai Y, Yang H, Xiong Q, Wang K, Zhou J, Li Y, Wang S (2020) A review of recent advances in biomass pyrolysis. Energy Fuels 34(12):15557-15578.

https://doi.org/10.1021/acs.energyfuels.0c03107

156. Wang J (2018) Reuse of Heavy Metal from Industrial Effluent Water. IOP Conference Series: Earth and Environmental Science, 199(4). https://doi.org/10.1088/1755-1315/199/4/042002

157. Wang LK, Vaccari DA, Li Y, Shammas NK (2005) Chemical Precipitation. In L.K Wang, T. Hung, Y, \& N.. Shammas (Eds.), Physicochemical Treatment Processes (1st ed., Vol. 3, pp. 141-197). The Humana Press Inc. https://doi.org/10.1385/1-59259-820-x:141

158. Wei Y, Wang Y, Zhang X, Xu T (2013) Comparative study on regenerating sodium hydroxide from the spent caustic by bipolar membrane electrodialysis (BMED) and electro- electrodialysis (EED). Sep Purif Technol 118:1-5. https://doi.org/10.1016/j.seppur.2013.06.025

159. Wikberg H, Jermakka J (2015) Hydrothermal refining of biomass - an overview and future perspectives Rights an overview and future perspectives. Tappi Journa 14(March):195-207. https://doi.org/10.32964/TJ14.3.195

160. Williams CJ (1992) Testing the performance of spool-wound cartridge filters. Filtration and Separation 29(2):162-168. https://doi.org/10.1016/0015-1882(92)80045-K

161. Wong JWC, Selvam A (2006) Speciation of heavy metals during co-composting of sewage sludge with lime. Chemosphere 63:980-986. https://doi.org/10.1016/j.chemosphere.2005.08.045

162. Woodard, Curran (2006a) Evaluating and Selecting Industrial Waste Treatment Systems. In ButterworthHeinemann (Ed.), Industrial Waste Treatment Handbook (2nd ed., pp. 1-28). Elsevier Science. https://doi.org/10.1016/B978-0-7506-7963-3.50003-5

163. Woodard, Curran (2006b) Methods for Treating Wastewaters from Industry. In Butterworth-Heinemann (Ed.), Industrial Waste Treatment Handbook (2nd ed., pp. 149-334). Elsevier Science. https://doi.org/10.1016/b978-075067963-3/50009-6

164. Woodard, Curran (2006c) Solid Waste Treatment and Disposal. In: Butterworth-Heinemann (ed) Industrial Waste Treatment Handbook, 2nd edn. Elsevier Science, pp 363-408

165. Wu D, Chen GQ, Hu B, Deng H (2019) Feasibility and energy consumption analysis of phenol removal from salty wastewater by electro-electrodialysis. Sep Purif Technol. https://doi.org/10.1016/j.seppur.2019.01.001

166. Xie M, Kyong H, Gray SR, Elimelech M (2016) Membrane-based processes for wastewater nutrient recovery: Technology, challenges, and future direction. Water Res 89:210-221. https://doi.org/10.1016/j.watres.2015.11.045 
167. Yadav A, Labhasetwar PK, Shahi VK (2022) Membrane distillation crystallization technology for zero liquid discharge and resource recovery: Opportunities, challenges and futuristic perspectives. Sci Total Environ 806:150692. https://doi.org/10.1016/j.scitotenv.2021.150692

168. Yadav B, Chavan S, Atmakuri A, Tyagi RD, Drogui P (2020) A review on recovery of proteins from industrial wastewaters with special emphasis on PHA production process: Sustainable circular bioeconomy process development. Bioresour Technol 317(August):124006.

https://doi.org/10.1016/j.biortech.2020.124006

169. Yadav G, Mishra A, Ghosh P, Sindhu R, Vinayak V, Pugazhendhi A (2021) Technical, economic and environmental feasibility of resource recovery technologies from wastewater. Sci Total Environ 796:149022. https://doi.org/10.1016/j.scitotenv.2021.149022

170. Yan T, Ye Y, Ma H, Zhang Y, Guo W, Du B, Wei Q, Wei D, Ngo HH (2018a) A critical review on membrane hybrid system for nutrient recovery from wastewater. Chem Eng J.

https://doi.org/10.1016/j.cej.2018.04.166

171. Yan T, Ye Y, Ma H, Zhang Y, Guo W, Du B, Wei Q, Wei D, Ngo HH (2018b) A critical review on membrane hybrid system for nutrient recovery from wastewater. Chem Eng J 348:143-156. https://doi.org/10.1016/j.cej.2018.04.166

172. Yang C, Hu Y, Cao L, Yang J (2003) Performance Optimization of an Electromembrane Reactor for Recycling and Resource Recovery of Desulfurization Residuals. VTT Publications 60(504):3-194. https://doi.org/10.1002/aic

173. Yang L, Hu W, Chang Z, Liu T, Fang D, Shao P, Shi H, Luo X (2021) Electrochemical recovery and high value-added reutilization of heavy metal ions from wastewater: Recent advances and future trends. Environment International, 152. https://doi.org/10.1016/j.envint.2021.106512

174. Ye Y, Ngo HH, Guo W (2020) Bioresource Technology Reports Nutrient recovery from wastewater: From technology to economy. Bioresource Technology Reports 11(April):100425. https://doi.org/10.1016/j.biteb.2020.100425

175. Yildiz Sizirici B (2012) Water and wastewater treatment: Biological processes. In F. Zeman (Ed.), Metropolitan Sustainability: Understanding and Improving the Urban Environment (1st ed., pp. 406428). Woodhead Publishing. https://doi.org/10.1533/9780857096463.3.406

176. Yoldi M, Fuentes-Ordoñez EG, Korili SA, Gil A (2019) Zeolite synthesis from industrial wastes. Microporous Mesoporous Mater 287(June):183-191. https://doi.org/10.1016/j.micromeso.2019.06.009

177. Yorkor B, Momoh Y (2019) A Review of Anoxic Wastewater Treatment: An Overlooked Aspect in A Review of Anoxic Wastewater Treatment : An Overlooked Aspect in Wastewater Treatment in Nigeria. https://doi.org/10.12691/ajwr-7-4-2. January

178. Yoshimura M, Byrappa K (2008) Hydrothermal Processing of Materials: Past, Present and Future. J Mater Sci 43(April):2085-2103. https://doi.org/10.1007/s10853-007-1853-x

179. Yoshino H, Hori T, Hosomi M, Terada A (2020) Identifying prokaryotes and eukaryotes disintegrated by a high-pressure jet device for excess activated sludge reduction. Biochem Eng J 157(January):107495. https://doi.org/10.1016/j.bej.2020.107495 
180. Zaharioiu AM, Bucura F, lonete RE, Marin F, Constantinescu M, Oancea S (2021) Opportunities regarding the use of technologies of energy recovery from sewage sludge. SN Applied Sciences 3(9). https://doi.org/10.1007/s42452-021-04758-3

181. Zhang T, Tremblay PL (2016) Editorial: Current challenges and future perspectives on emerging bioelectrochemical technologies. Front Microbiol 7(JUN):8-10. https://doi.org/10.3389/fmicb.2016.00860

182. Zhao C, Zhou J, Yan Y, Yang L, Xing G, Li H, Wu P, Wang M, Zheng H (2021) Application of coagulation/flocculation in oily wastewater treatment: A review. Sci Total Environ 765:142795. https://doi.org/10.1016/j.scitotenv.2020.142795

183. Zhao L, Gu W, He P, Shao L (2010) Effect of air-flow rate and turning frequency on bio-drying of dewatered sludge. Water Res 44(20):6144-6152. https://doi.org/10.1016/j.watres.2010.07.002

184. Zhao R, Satpradit O, Rijnaarts HHM, Biesheuvel PM, van der Wal A (2013) Optimization of salt adsorption rate in membrane capacitive deionization. Water Res 47(5):1941-1952. https://doi.org/10.1016/j.watres.2013.01.025

185. Zheng M, Sun Z, Han H, Zhang Z, Ma W, Xu C (2021) Enhanced coagulation coupled with heavy metal capturing for heavy metals removal from coal gasification brine and a novel mathematical model. Journal of Water Process Engineering 40(February):101954. https://doi.org/10.1016/j.jwpe.2021.101954

\section{Tables}


Table 1

Conventional Physicochemical treatment units: Advantages, disadvantages and gaps/potential to exploit.

\begin{tabular}{|c|c|c|c|c|}
\hline Technology & $\begin{array}{l}\text { Advantages }(\sqrt{ }) \text {; Disadvantages }(\mathrm{x}) \text {; } \\
\text { Gaps/Potential to exploit }(\bullet) \text {. }\end{array}$ & Category & $\begin{array}{l}\text { Common or } \\
\text { Potencial } \\
\text { Industry } \\
\text { Sector }\end{array}$ & Source \\
\hline $\begin{array}{l}\text { Coagulation/ } \\
\text { Flocculation } \\
\text { (conventional) }\end{array}$ & $\begin{array}{l}\sqrt{ } \text { Improves sludge stability. } \\
\sqrt{ } \text { Improves sedimentation rates. } \\
\sqrt{ } \text { Extensive large-scale experience. } \\
\sqrt{ } \text { Simple operation. } \\
\sqrt{ } \text { Significant decrease in COD } \\
\text { (Depending on the content in } \\
\text { solids). } \\
\sqrt{ } \text { Efficient in removing total solid } \\
\text { suspend and colloidal material. } \\
\sqrt{ } \text { Low CAPEX (capital expenditure). } \\
\text { x Reagent dosage requirement. } \\
x \text { Requirement of additional } \\
\text { operations for agglomerate solids } \\
\text { removal. } \\
x \text { Additional costs for the treatment } \\
\text { of the sludge. } \\
x \text { The requirement for flocculating } \\
\text { agents can change the chemistry of } \\
\text { the water. }\end{array}$ & Established & $\begin{array}{l}\text { Electronic } \\
\text { Nuclear } \\
\text { Petroleum } \\
\text { Pulp and } \\
\text { Paper } \\
\text { Tannery }\end{array}$ & $\begin{array}{l}\text { (Abdullah et } \\
\text { al., 2019; } \\
\text { Bhandari \& } \\
\text { Ranade, } \\
\text { 2014; } \\
\text { Dayarathne } \\
\text { et al., 2021; } \\
\text { Mels \& } \\
\text { Teerikangas, } \\
\text { 2002; Ranade } \\
\text { \& Bhandari, } \\
\text { 2014b; } \\
\text { Varjani et al., } \\
\text { 2020; G. } \\
\text { Yadav et al., } \\
\text { 2021) }\end{array}$ \\
\hline
\end{tabular}




\section{Technology Advantages $(\sqrt{ })$; Disadvantages $(x)$; $\quad$ Category Gaps/Potential to exploit $(\bullet)$.

- New coagulants with specific properties to treat IWW.

- New inorganic coagulants such as polymers, thus they may provide a reduction in treatment dosages, and thereby mitigating some of the toxicity issues.

- New organic coagulants from wastes.

- Integration of advantages of inorganic and organic coagulants in the form of coagulant formulation (physical or chemical functional modification).

- More information in the open literature on Coagulation/ Flocculation viability in IWW treatment and techno-economic for feasibility analysis.

- Research is still required to better understand how coagulant systems can be better optimized for various conditions.

- Explore opportunities to improve efficiency in coagulant systems by better understanding the control parameters.

- New designs and theoretical models for predicting performance.

- There is a need to develop more biodegradable polymer coagulants for use in the IWW treatment to reduce issues associated with toxic and hard-to-treat sludge.

- Performance guarantees for commercial operations involving different IWW and a variety of pollutants.

$\sqrt{ }$ Easy to operate.

$\sqrt{ }$ Low OPEX(Operational expenditures) and CAPEX.

$\sqrt{ }$ Extensive large-scale experience.
Established Nuclear

Pulp and

Paper

Tannery
(Mels \& Teerikangas, 2002; Ødegaard et al., 2002; G. Yadav et al., 2021) 


\section{Technology $\quad$ Advantages $(\sqrt{ })$; Disadvantages $(x)$; $\quad$ Category Gaps/Potential to exploit $(\bullet)$.

$x$ Depending on the pollutant, it may not be efficient.

$x$ Very area-consuming.

x Mature with little potential less to exploit.

- New designs and theoretical

models for predicting performance.

- Less space requirement.

- Reduce hydraulic retention times.

- Reuse for the sludge produced.

Flotation

$\sqrt{ }$ High selectivity of metals.

$\sqrt{ }$ Low OPEX.

$\sqrt{ }$ Extensive large-scale experience.

$\sqrt{ }$ High removal rate, even of small and low-density particles.

$\sqrt{ }$ More concentrated sludge, which means low volume to be treated.

$\sqrt{ }$ Elimination of certain volatile compounds.

$\sqrt{ }$ Good selectivity and easy construction systems.

$\sqrt{ }$ Low hydraulic retention time.

$\sqrt{ }$ Potential use in water recovery systems, such as metal recovery.

$x$ Low recovery rate at high metal concentrations.

$x$ Requirement of surfactant agents to improve performance.

$x$ Environmental toxicity limits its wide application in the environmental pollution remediation field.

$x$ Additional costs for the treatment of the sludge.
Established Battery

Electronic

Petroleum

Pulp and

Paper

Tannery
(Kyzas, 2018; Mels \& Teerikangas, 2002; Patil et al., 2016; Peng et al., 2019; Varjani et al., 2020; G. Yadav et al., 2021) 


\begin{tabular}{|c|c|c|c|c|}
\hline Technology & $\begin{array}{l}\text { Advantages }(\sqrt{ }) \text {; Disadvantages }(\mathrm{x}) \text {; } \\
\text { Gaps/Potential to exploit }(\bullet) \text {. }\end{array}$ & Category & $\begin{array}{l}\text { Common or } \\
\text { Potencial } \\
\text { Industry } \\
\text { Sector }\end{array}$ & Source \\
\hline & $\begin{array}{l}\text { - Even though the progress obtained } \\
\text { in the exploration of green, efficient } \\
\text { surfactants as collectors for } \\
\text { flotation. There are still a series of } \\
\text { challenges to making this } \\
\text { technology an extensively industrial } \\
\text { application. }\end{array}$ & & & \\
\hline & $\begin{array}{l}\text { - Development of new } \\
\text { biosurfactants with an } \\
\text { environmentally friendly feature, } \\
\text { even in large dosages, and also } \\
\text { short flotation time, and high } \\
\text { efficiency. }\end{array}$ & & & \\
\hline & $\begin{array}{l}\text { - Development of Nanoparticle as } \\
\text { new collectors. }\end{array}$ & & & \\
\hline & $\begin{array}{l}\text { - Lowering costs and scalable } \\
\text { production research is still needed. }\end{array}$ & & & \\
\hline & $\begin{array}{l}\text { - Performance guarantees for } \\
\text { commercial operations involving } \\
\text { different IWW and a variety of } \\
\text { pollutants. }\end{array}$ & & & \\
\hline \multirow[t]{10}{*}{ Screening } & $\sqrt{ }$ Extensive large-scale experience. & Established & Agriculture & \multirow{10}{*}{$\begin{array}{l}\text { (Ljunggren, } \\
\text { 2006; Mels \& } \\
\text { Teerikangas, } \\
\text { 2002) }\end{array}$} \\
\hline & $\sqrt{ }$ Simple operation. & & Beverage & \\
\hline & $\begin{array}{l}\sqrt{ } \text { It does not change the chemistry } \\
\text { of the water. }\end{array}$ & & \multirow[t]{8}{*}{ Food } & \\
\hline & $\sqrt{ }$ Low OPEX and CAPEX. & & & \\
\hline & $\begin{array}{l}\text { x Limited to relatively large } \\
\text { suspended solid material. }\end{array}$ & & & \\
\hline & $\begin{array}{l}x \text { Irreversible clogging of the filter } \\
\text { media. }\end{array}$ & & & \\
\hline & $\begin{array}{l}\text { x Mature with little potential less to } \\
\text { exploit. }\end{array}$ & & & \\
\hline & $\begin{array}{l}\text { - There is a need for more } \\
\text { documentation. }\end{array}$ & & & \\
\hline & $\begin{array}{l}\text { - Development of a stronger } \\
\text { theoretical basis. }\end{array}$ & & & \\
\hline & - Reuse for the sludge produced. & & & \\
\hline
\end{tabular}




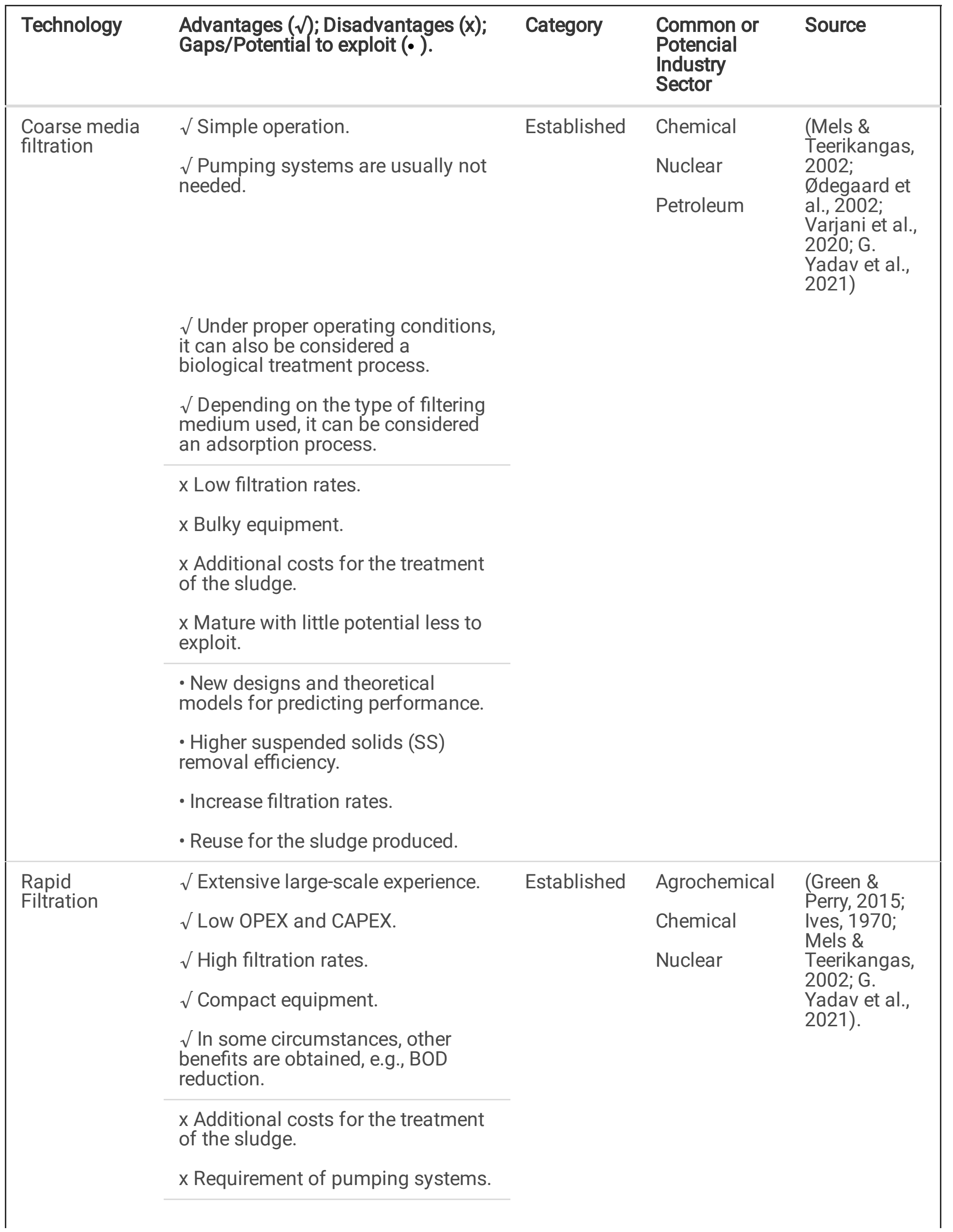




\begin{tabular}{|c|c|c|c|c|}
\hline Technology & $\begin{array}{l}\text { Advantages }(\sqrt{ }) \text {; Disadvantages }(\mathrm{x}) \text {; } \\
\text { Gaps/Potential to exploit }(\bullet) \text {. }\end{array}$ & Category & $\begin{array}{l}\text { Common or } \\
\text { Potencial } \\
\text { Industry } \\
\text { Sector }\end{array}$ & Source \\
\hline & $\begin{array}{l}\text { - New designs and theoretical } \\
\text { models for predicting performance. } \\
\text { - Higher SS removal efficiency. } \\
\text { - Increase filtration rates. } \\
\text { - Reduce the requirement of } \\
\text { pumping systems. } \\
\text { - Reuse for the cake produced. }\end{array}$ & & & \\
\hline $\begin{array}{l}\text { Cartridge } \\
\text { filters (CF) }\end{array}$ & $\begin{array}{l}\sqrt{ } \text { Extensive large-scale experience. } \\
\sqrt{ } \text { Simple operation. } \\
\sqrt{ } \text { Low CAPEX and OPEX. } \\
\sqrt{ } \text { Modular equipment, it is easily } \\
\text { scalable. } \\
\sqrt{ } \text { Low or no impact on water } \\
\text { chemistry. } \\
\text { x Constant change requirement. } \\
\text { x Limited to certain particle size. } \\
\text { - Membranes with chemical } \\
\text { resistance. } \\
\text { - Temperature resistance. } \\
\text { - More sustainable manufacturing } \\
\text { membranes. } \\
\text { - Reusability. } \\
\text { - Recovery of solids. }\end{array}$ & Established & $\begin{array}{l}\text { Chemical } \\
\text { Mining } \\
\text { Nuclear } \\
\text { Petroleum } \\
\text { Textile }\end{array}$ & $\begin{array}{l}\text { (Farhat et al., } \\
\text { 2020; } \\
\text { Williams, } \\
\text { 1992; G. } \\
\text { Yadav et al., } \\
\text { 2021) }\end{array}$ \\
\hline
\end{tabular}




\begin{tabular}{|c|c|c|c|c|}
\hline Technology & $\begin{array}{l}\text { Advantages }(\sqrt{ }) \text {; Disadvantages }(\mathrm{x}) \text {; } \\
\text { Gaps/Potential to exploit }(\cdot) \text {. }\end{array}$ & Category & $\begin{array}{l}\text { Common or } \\
\text { Potencial } \\
\text { Industry } \\
\text { Sector }\end{array}$ & Source \\
\hline Precipitation & $\begin{array}{l}\sqrt{ } \text { Low CAPEX. } \\
\sqrt{ } \text { Extensive large-scale experience. } \\
\sqrt{ } \text { Low OPEX (except oxidative } \\
\text { precipitation). } \\
\sqrt{ } \text { Simple operation. } \\
\sqrt{ } \text { High recovery (depends on the } \\
\text { chemical agent and the species). } \\
\sqrt{ } \text { The widely used method in the } \\
\text { industry. } \\
\sqrt{ } \text { Selective recovery (depends on } \\
\text { the species). } \\
\sqrt{ } \text { Potential use in water recovery } \\
\text { systems. } \\
\text { x Additional costs for the treatment } \\
\text { of the sludge. } \\
x \text { Reagent dosage requirement. } \\
\text { x It changes the chemistry of the } \\
\text { water. } \\
x \text { Requirement of an oxidation stage } \\
\text { for some complex metals. } \\
\text { x Inviable at very low } \\
\text { concentrations. } \\
\text { - Understanding the precipitation } \\
\text { mechanism from different } \\
\text { wastewater industry scenarios. } \\
\text { - Increase precipitation rates. } \\
\text { - Reduce space requirement. } \\
\text { - Reuse for the sludge produced. }\end{array}$ & Established & $\begin{array}{l}\text { Agrochemical } \\
\text { Battery } \\
\text { Chemical } \\
\text { Electronic } \\
\text { Metal } \\
\text { Mining } \\
\text { Tannery }\end{array}$ & $\begin{array}{l}\text { (Abdullah et } \\
\text { al., 2019; } \\
\text { Chen et al., } \\
\text { 2017; Mels \& } \\
\text { Teerikangas, } \\
\text { 2002; } \\
\text { Mocellin et } \\
\text { al., 2017; } \\
\text { Patil et al., } \\
\text { 2016; Roy \& } \\
\text { Bhattacharya, } \\
\text { 2014; } \\
\text { Sayilgan et } \\
\text { al., 2010) }\end{array}$ \\
\hline
\end{tabular}




\begin{tabular}{|c|c|c|c|c|}
\hline Technology & $\begin{array}{l}\text { Advantages }(\sqrt{ }) \text {; Disadvantages }(\mathrm{x}) \text {; } \\
\text { Gaps/Potential to exploit }(\bullet) \text {. }\end{array}$ & Category & $\begin{array}{l}\text { Common or } \\
\text { Potencial } \\
\text { Industry } \\
\text { Sector }\end{array}$ & Source \\
\hline Adsorption & $\begin{array}{l}\sqrt{ } \text { Simple operation. } \\
\sqrt{ } \text { Extensive large-scale experience. } \\
\sqrt{ } \text { It is possible to regenerate the } \\
\text { adsorbent surface. } \\
\sqrt{ } \text { Wide range of contaminants that } \\
\text { can be removed/recovered. } \\
\sqrt{ } \text { Possibility of selectivity } \\
\text { depending on the pollutant. } \\
\sqrt{ } \text { Relatively low OPEX. } \\
\sqrt{ } \text { Wide selection of adsorbents. } \\
\sqrt{ } \text { High research interest. } \\
\text { x Low-cost or natural adsorbents } \\
\text { have low removal performance. } \\
\text { x Inviable at high concentrations. } \\
x \text { Relatively high CAPEX. } \\
x \text { Low removal efficiency/cost ratio. } \\
x \text { Post-treatment processes are } \\
\text { required. }\end{array}$ & $\begin{array}{l}\text { Adaptive } \\
\text { Use }\end{array}$ & $\begin{array}{l}\text { Agricultural } \\
\text { Mining } \\
\text { Petroleum } \\
\text { Petroleum } \\
\text { Pulp and } \\
\text { Paper } \\
\text { Tannery } \\
\text { Textile }\end{array}$ & $\begin{array}{l}\text { (Abdullah et } \\
\text { al., 2019; ;l- } \\
\text { Rashdi et al., } \\
\text { 2011; } \\
\text { Bhandari \& } \\
\text { Ranade, } \\
\text { 2014; Mels \& } \\
\text { Teerikangas, } \\
\text { 2002; Patil et } \\
\text { al., 2016; } \\
\text { Perez et al., } \\
\text { 2019; Ranade } \\
\text { \& Bhandari, } \\
\text { 2014b; } \\
\text { Shrestha et } \\
\text { al., 2021; G. } \\
\text { Yadav et al., } \\
\text { 2021) }\end{array}$ \\
\hline
\end{tabular}




\section{Technology Advantages $(\sqrt{ })$; Disadvantages $(x)$; $\quad$ Category Gaps/Potential to exploit $(\bullet)$.

- Nanomaterial and/or polymer as adsorbents.

- New adsorbents from biomass and wastes.

- In general, new, efficient, and costeffective adsorbents for better performance.

- High capacity for removal of pollutants with selectivity.

- Efficient ways to regenerate and reuse adsorbents (fewer chemicals and energy and avoid producing secondary waste streams).

- Better theoretical models for prediction of process performance and evaluation.

- Performance guarantees for commercial operations involving different IWW and a variety of pollutants.

- A balance between capacity and renewability must be met to transcend from lab-scale experiments to pilot-scale studies for new adsorbents.

Absorption $\quad \sqrt{ }$ High efficiency in removal of metallic species.

$\sqrt{ }$ Allows selective removal of metals.

$\sqrt{ }$ It is possible to regenerate the absorbent solution.

$\sqrt{ }$ Extensive large-scale experience.

$\sqrt{ }$ Relatively low CAPEX.
Adaptive Chemical

Use
(Biswas et al., 2016; Innocenzi \& Veglio, 2012; Ranade \& Bhandari, 2014b, 2014a) 


\section{Technology $\quad$ Advantages $(\sqrt{ })$; Disadvantages $(x)$; $\quad$ Category Gaps/Potential to exploit $(\bullet)$.

$x$ Requirement of a complementary process for the removal/recovery of pollutants.

x Relatively high OPEX.

x Inviable at low pollutant concentrations.

$x$ Excessive use of extractants.

$x$ Other risks associated with organic extracts.

x Limited application in wastewater.

- New biodegradable solvents, suitable for selective removal of pollutants.

- New equipment and process design for extraction and recovery.

- Reduce costs.

- Efficient ways to regenerate and reuse absorbents (less energy and avoid producing secondary waste streams).

- Performance guarantees for commercial operations involving different IWW and a variety of pollutants.

- A better understanding of the process and enhanced physical property database. 


\begin{tabular}{|c|c|c|c|c|}
\hline Technology & $\begin{array}{l}\text { Advantages }(\sqrt{ }) \text {; Disadvantages }(\mathrm{x}) \text {; } \\
\text { Gaps/Potential to exploit }(\bullet) \text {. }\end{array}$ & Category & $\begin{array}{l}\text { Common or } \\
\text { Potencial } \\
\text { Industry } \\
\text { Sector }\end{array}$ & Source \\
\hline Ion Exchange & $\begin{array}{l}\sqrt{ } \text { Extensive large-scale experience. } \\
\sqrt{ } \text { An effective system for the } \\
\text { recovery of metals. } \\
\sqrt{ } \text { The system has a high economic } \\
\text { and energy efficiency. } \\
\sqrt{ } \text { Simple operation } \\
\sqrt{ } \text { There is no generation of sludge. } \\
\sqrt{ } \text { Fast and efficient process. } \\
\sqrt{ } \text { Can become selective with some } \\
\text { pollutants. } \\
x \text { Resins with limited adsorption } \\
\text { capacity. } \\
x \text { Risk of system clogging. } \\
x \text { Risk of degradation of the resins } \\
\text { depending on the quality of the } \\
\text { wastewater. } \\
x \text { Generally used in solutions with } \\
\text { low metal concentrations. } \\
x \text { Large amounts of chemical } \\
\text { agents are required for the } \\
\text { regeneration of exchange resins. } \\
x \text { The regeneration of resins } \\
\text { generates multiple secondary } \\
\text { pollution problems. } \\
x \text { Commercial resins are not } \\
\text { selective. } \\
x \text { Not effective for emerging } \\
\text { pollutants. } \\
x \text { High CAPEX and OPEX. }\end{array}$ & $\begin{array}{l}\text { Adaptive } \\
\text { Use }\end{array}$ & $\begin{array}{l}\text { Agrochemical } \\
\text { Battery } \\
\text { Chemical } \\
\text { Nuclear } \\
\text { Textile }\end{array}$ & $\begin{array}{l}\text { (Abdullah et } \\
\text { al., 2019; } \\
\text { Bhandari \& } \\
\text { Ranade, } \\
\text { 2014; Mels \& } \\
\text { Teerikangas, } \\
\text { 2002; Patil et } \\
\text { al., 2016; Roy } \\
\text { \& } \\
\text { Bhattacharya, } \\
\text { 2014; G. } \\
\text { Yadav et al., } \\
\text { 2021) }\end{array}$ \\
\hline
\end{tabular}




\begin{tabular}{|c|c|c|c|c|}
\hline Technology & $\begin{array}{l}\text { Advantages }(\sqrt{ }) \text {; Disadvantages }(\mathrm{x}) \text {; } \\
\text { Gaps/Potential to exploit }(\bullet) \text {. }\end{array}$ & Category & $\begin{array}{l}\text { Common or } \\
\text { Potencial } \\
\text { Industry } \\
\text { Sector }\end{array}$ & Source \\
\hline & $\begin{array}{l}\text { - Development of low-cost ion } \\
\text { exchange materials. }\end{array}$ & & & \\
\hline & $\begin{array}{l}\text { - Devise more robust, high-capacity, } \\
\text { and high-selectivity exchangers. }\end{array}$ & & & \\
\hline & $\begin{array}{l}\text { - Development of highly selective } \\
\text { materials. }\end{array}$ & & & \\
\hline & $\begin{array}{l}\text { - More experimental and theoretical } \\
\text { information of sorption equilibria is } \\
\text { required. }\end{array}$ & & & \\
\hline & $\begin{array}{l}\text { - More information in the open } \\
\text { literature on adsorption/ion } \\
\text { exchange in IWW treatment and } \\
\text { techno-economic for feasibility } \\
\text { analysis. }\end{array}$ & & & \\
\hline \multirow{12}{*}{$\begin{array}{l}\text { Multi-effect } \\
\text { evaporative } \\
\text { crystallization } \\
\text { (MEEC) }\end{array}$} & $\sqrt{ }$ Extensive large-scale experience. & \multirow[t]{12}{*}{ Established } & \multirow[t]{12}{*}{ Chemical } & \multirow{12}{*}{$\begin{array}{l}\text { (De Luna et } \\
\text { al., 2017; } \\
\text { Fernández- } \\
\text { Torres et al. } \\
\text { 2012; Lu et } \\
\text { al., 2017) }\end{array}$} \\
\hline & $\begin{array}{l}\sqrt{ } \text { It allows the recovery of } \\
\text { substances of interest with high } \\
\text { purity. }\end{array}$ & & & \\
\hline & $\sqrt{ }$ High processing speed. & & & \\
\hline & $\begin{array}{l}\sqrt{ } \text { Potential use in water recovery } \\
\text { systems. }\end{array}$ & & & \\
\hline & x High energy requirement. & & & \\
\hline & x Relatively complex system. & & & \\
\hline & x High OPEX and CAPEX. & & & \\
\hline & $\begin{array}{l}\text { - In IWW treatment processes, the } \\
\text { long-term reliability has not } \\
\text { completely been proven. }\end{array}$ & & & \\
\hline & $\begin{array}{l}\text { - Better configurations should be } \\
\text { developed to improve the heat } \\
\text { transfer coefficients. Thus, reduce } \\
\text { OPEX and CAPEX. }\end{array}$ & & & \\
\hline & - Reduce complexity. & & & \\
\hline & $\begin{array}{l}\text { - Residual-heat integration in the } \\
\text { industry. }\end{array}$ & & & \\
\hline & • Hybrid processes. & & & \\
\hline
\end{tabular}




\begin{tabular}{|c|c|c|c|c|}
\hline Technology & $\begin{array}{l}\text { Advantages }(\sqrt{ }) \text {; Disadvantages }(\mathrm{x}) \text {; } \\
\text { Gaps/Potential to exploit }(\bullet) \text {. }\end{array}$ & Category & $\begin{array}{l}\text { Common or } \\
\text { Potencial } \\
\text { Industry } \\
\text { Sector }\end{array}$ & Source \\
\hline $\begin{array}{l}\text { Mechanical } \\
\text { vapor } \\
\text { recompression } \\
\text { crystallization } \\
\text { (MVRC) }\end{array}$ & $\begin{array}{l}\sqrt{ } \text { Extensive large-scale experience. } \\
\sqrt{ } \text { High thermodynamic efficiency. } \\
\sqrt{ } \text { It allows the recovery of } \\
\text { substances of interest with high } \\
\text { purity. } \\
\sqrt{ } \text { Configuration of relatively } \\
\text { compact equipment. } \\
\sqrt{ } \text { Potential use in water recovery } \\
\text { systems. } \\
\text { x High energy requirement. } \\
\text { x High OPEX and CAPEX. } \\
\text { - In IWW treatment processes, the } \\
\text { long-term reliability has not } \\
\text { completely been proven. } \\
\text { - Better configurations should be } \\
\text { developed to improve the heat } \\
\text { transfer coefficients. Thus, reduce } \\
\text { OPEX and CAPEX. } \\
\text { - Residual-heat integration in the } \\
\text { industry. }\end{array}$ & Established & Chemical & $\begin{array}{l}\text { (De Luna et } \\
\text { al., 2017; } \\
\text { Fernández- } \\
\text { Torres et al., } \\
\text { 2012; Lu et } \\
\text { al., 2017) }\end{array}$ \\
\hline $\begin{array}{l}\text { Solute } \\
\text { crystallization } \\
\text { (SC) }\end{array}$ & $\begin{array}{l}\sqrt{ } \text { Widely used system in salt } \\
\text { remotion. } \\
\sqrt{ } \text { Simple operation. } \\
\sqrt{ } \text { It allows the recovery of } \\
\text { substances of interest with high } \\
\text { purity. } \\
\sqrt{ } \text { Potential use in water recovery } \\
\text { systems. } \\
\text { x Cooling systems requirement. } \\
\text { x Limited treatment capacity. } \\
\text { x High OPEX and CAPEX. }\end{array}$ & Established & Chemical & $\begin{array}{l}\text { (De Luna et } \\
\text { al., 2017; Lu } \\
\text { et al., 2017) }\end{array}$ \\
\hline
\end{tabular}




\section{Technology $\quad$ Advantages $(\sqrt{ })$; Disadvantages $(x)$; $\quad$ Category Gaps/Potential to exploit $(\bullet)$.

- In IWW treatment processes, the long-term reliability has not completely been proven.

- Better configurations should be developed to improve the heat transfer coefficients. Thus, reduce OPEX and CAPEX.

- Hybrid processes.

Freeze crystallization (FC) $\sqrt{ }$ Simple operation.

$\sqrt{ }$ It allows the recovery of substances of interest with high purity.

$\sqrt{ }$ Potential use in water recovery systems.

x Cooling systems requirement.

$x$ Not extensive large-scale experience.

$x$ Difficulty in recovering the solid.

x Limited treatment capacity.

$x$ High OPEX and CAPEX.

- In IWW treatment processes, the long-term reliability has not completely been proven.

- Better configurations should be developed to improve the heat transfer coefficients. Thus, reduce OPEX and CAPEX.

- Performance guarantees for commercial operations involving different IWW and a variety of pollutants.

- Hybrid processes. 


\begin{tabular}{|c|c|c|c|c|}
\hline Technology & $\begin{array}{l}\text { Advantages }(\sqrt{ }) \text {; Disadvantages }(\mathrm{x}) \text {; } \\
\text { Gaps/Potential to exploit }(\bullet) \text {. }\end{array}$ & Category & $\begin{array}{l}\text { Common or } \\
\text { Potencial } \\
\text { Industry } \\
\text { Sector }\end{array}$ & Source \\
\hline \multirow[t]{12}{*}{ Evaporation } & $\sqrt{ }$ Simple operation. & \multirow[t]{12}{*}{ Established } & Chemical & \multirow{12}{*}{$\begin{array}{l}\text { (Ahirrao, } \\
\text { 2014; G. } \\
\text { Yadav et al., } \\
\text { 2021) }\end{array}$} \\
\hline & $\begin{array}{l}\sqrt{ } \text { Well-established unit operation in } \\
\text { the industry. }\end{array}$ & & \multirow[t]{11}{*}{ Nuclear } & \\
\hline & $\sqrt{ }$ Extensive large-scale experience. & & & \\
\hline & $\begin{array}{l}\sqrt{ } \text { Potential use in Zero liquid } \\
\text { discharge scheme. }\end{array}$ & & & \\
\hline & $x$ High OPEX and CAPEX. & & & \\
\hline & x High energy requirement. & & & \\
\hline & $x$ Prone to Fouling. & & & \\
\hline & $\begin{array}{l}x \text { Inviable for selective recovery of } \\
\text { some substances. }\end{array}$ & & & \\
\hline & $\begin{array}{l}\mathrm{x} \text { Mature technology with little } \\
\text { potential less to exploit. }\end{array}$ & & & \\
\hline & $\begin{array}{l}\text { - Better configurations should be } \\
\text { developed to improve the heat } \\
\text { transfer coefficients. Thus, reduce } \\
\text { OPEX and CAPEX. }\end{array}$ & & & \\
\hline & $\begin{array}{l}\text { - Cleaning in place (CIP) systems } \\
\text { could be improved. }\end{array}$ & & & \\
\hline & $\begin{array}{l}\text { - Residual-heat integration in the } \\
\text { industry. }\end{array}$ & & & \\
\hline \multirow[t]{7}{*}{ Stripping } & $\sqrt{ }$ Simple operation. & \multirow[t]{7}{*}{ Established } & Chemical & \multirow{7}{*}{$\begin{array}{l}\text { (Kinidi et al., } \\
\text { 2018; Maurer } \\
\text { et al., 2002; } \\
\text { Srinivasan et } \\
\text { al., 2008; } \\
\text { Varjani et al., } \\
\text { 2020) }\end{array}$} \\
\hline & $\begin{array}{l}\sqrt{ } \text { Simple remotion of gaseous } \\
\text { pollutants. }\end{array}$ & & \multirow[t]{6}{*}{ Petroleum } & \\
\hline & $\sqrt{ }$ Extensive large-scale experience. & & & \\
\hline & x Cooling systems requirement. & & & \\
\hline & x Difficulty recovering pollutants. & & & \\
\hline & x Limited treatment capacity. & & & \\
\hline & $x$ High CAPEX. & & & \\
\hline
\end{tabular}


- Hybrid processes with membrane contactors, membrane distillation, microwave, etc.

- Structure optimization.

- Energy self-sufficient, in some new configurations.

- Performance guarantees for commercial operations involving different IWW and a variety of pollutants. 
Table 2

Unconventional Physicochemical treatment units: Advantages, disadvantages and gaps/potential to exploit.

\begin{tabular}{|c|c|c|c|c|}
\hline Technology & $\begin{array}{l}\text { Advantages }(\sqrt{ }) \text {; } \\
\text { Disadvantages }(x) \text {; } \\
\text { Gaps/Potential to } \\
\text { exploit }(\cdot) \text {. }\end{array}$ & Category & $\begin{array}{l}\text { Common or Potencial } \\
\text { Industry Sector }\end{array}$ & Source \\
\hline \multirow{14}{*}{$\begin{array}{l}\text { Advanced } \\
\text { Oxidation (AO) }\end{array}$} & \multirow{4}{*}{$\begin{array}{l}\sqrt{ } \text { Extensive large-scale } \\
\text { experience. } \\
\sqrt{ } \text { Fast reaction kinetics, } \\
\text { so retention hydraulic } \\
\text { times are very low. }\end{array}$} & \multirow[t]{14}{*}{ Emerging } & \multirow{14}{*}{$\begin{array}{l}\text { Agriculture } \\
\text { Electronic } \\
\text { Medical } \\
\text { Petroleum } \\
\text { Petroleum } \\
\text { Pharmaceutical } \\
\text { Tannery }\end{array}$} & \multirow{14}{*}{$\begin{array}{l}\text { (Bhandari \& } \\
\text { Ranade, } \\
\text { 2014; Bhuta, } \\
\text { 2014; Bottrel } \\
\text { et al., 2014; } \\
\text { Giwa et al., } \\
\text { 2021; } \\
\text { Helmreich \& } \\
\text { Metzger, } \\
\text { 2017; Varjani } \\
\text { et al., 2020; } \\
\text { Vogelpohl, } \\
\text { 2002; G. } \\
\text { Yadav et al., } \\
\text { 2021) }\end{array}$} \\
\hline & & & & \\
\hline & & & & \\
\hline & & & & \\
\hline & \multirow{4}{*}{$\begin{array}{l}\sqrt{ } \text { Low environmental } \\
\text { impact } \\
\sqrt{ } \text { If it is completed, it } \\
\text { does not introduce new } \\
\text { pollutants. } \\
\sqrt{ } \text { Can treat almost all } \\
\text { organic compounds } \\
\text { and can remove some } \\
\text { heavy metals. }\end{array}$} & & & \\
\hline & & & & \\
\hline & & & & \\
\hline & & & & \\
\hline & $\begin{array}{l}\sqrt{ } \text { It is also considered a } \\
\text { disinfection process. }\end{array}$ & & & \\
\hline & $\begin{array}{l}\sqrt{ } \text { There is no } \\
\text { generation of sludge. }\end{array}$ & & & \\
\hline & $\begin{array}{l}\sqrt{ } \text { It does not } \\
\text { concentrate pollutants, } \\
\text { so it does not usually } \\
\text { require post-treatment. }\end{array}$ & & & \\
\hline & $\begin{array}{l}\sqrt{ } \text { Removal of color and } \\
\text { odor. }\end{array}$ & & & \\
\hline & $\begin{array}{l}\sqrt{ } \text { Potential use in water } \\
\text { recycling systems. }\end{array}$ & & & \\
\hline & $\begin{array}{l}\sqrt{ } \text { Less space is } \\
\text { required in comparison } \\
\text { with conventional } \\
\text { treatment such as } \\
\text { biological treatment. }\end{array}$ & & & \\
\hline
\end{tabular}


Gaps/Potential to

exploit ( $)$.

$x$ High CAPEX and

OPEX.

$x$ Requirement of reactants and/or catalysts.

$x$ Pretreatment is essential to be efficient.

$x$ Formation of unknown intermediate compounds.

$x$ Depending on subsequent units or the use of water, the remaining oxidizing agent must be removed.

- Absence of awareness and understanding about the existing advanced oxidation technologies and their applications.

-Database of successful AO cases at lab, pilot, and plantlevel should be prepared.

- Reduce the cost of materials and processes.

- Hybrid processes and process intensification that result in enhanced efficiency, efficacy, and sustainability, such as OA/biological integration.

- Development of solar photocatalytic processes, with a focus on maximizing visible light utilization and minimizing catalyst deactivation.

- Hydrogen generation. 


\begin{tabular}{|c|c|c|c|c|}
\hline Technology & $\begin{array}{l}\text { Advantages }(\sqrt{ }) ; \\
\text { Disadvantages }(x) \text {; } \\
\text { Gaps/Potential to } \\
\text { exploit }(\cdot) \text {. }\end{array}$ & Category & $\begin{array}{l}\text { Common or Potencial } \\
\text { Industry Sector }\end{array}$ & Source \\
\hline & $\begin{array}{l}\text { - Waste minimization. } \\
\text { - Identification of } \\
\text { catalysts that can } \\
\text { address the issue of } \\
\text { sludge generation. } \\
\text { - Study the } \\
\text { combination of AO and } \\
\text { conventional treatment } \\
\text { processes such as } \\
\text { biological to reduce } \\
\text { OPEX. } \\
\text { - Use of up-to-date } \\
\text { reaction engineering } \\
\text { technologies for more } \\
\text { efficient and effective } \\
\text { AO systems. } \\
\text { - In IWW treatment } \\
\text { processes, the long- } \\
\text { term reliability has not } \\
\text { completely been } \\
\text { proven. } \\
\text { - Performance } \\
\text { guarantees for } \\
\text { commercial operations } \\
\text { involving different IWW } \\
\text { and a variety of } \\
\text { pollutants. } \\
\text { - Artificial neural } \\
\text { networks for modeling } \\
\text { of AO. } \\
\text { - Plasma activation in } \\
\text { photocatalysis } \\
\text { reactions. } \\
\text { - Development of } \\
\text { catalyst structures and } \\
\text { preparation. } \\
\text { processes. }\end{array}$ & & & \\
\hline
\end{tabular}




\begin{tabular}{|c|c|c|c|c|}
\hline Technology & $\begin{array}{l}\text { Advantages }(\sqrt{ }) \text {; } \\
\text { Disadvantages }(x) \text {; } \\
\text { Gaps/Potential to } \\
\text { exploit }(\cdot) \text {. }\end{array}$ & Category & $\begin{array}{l}\text { Common or Potencial } \\
\text { Industry Sector }\end{array}$ & Source \\
\hline $\begin{array}{l}\text { Electrocoagulation } \\
(\mathrm{EC})\end{array}$ & $\begin{array}{l}\sqrt{ } \text { Improves sludge } \\
\text { stability. } \\
\sqrt{ } \text { Improves } \\
\text { sedimentation rates. } \\
\sqrt{ } \text { Extensive large-scale } \\
\text { experience. } \\
\sqrt{ } \text { Does not require } \\
\text { dosing of } \\
\text { coagulants/flocculants. } \\
\sqrt{ } \text { Given the nature of } \\
\text { its application, the } \\
\text { amount of sludge } \\
\text { generated is lower than } \\
\text { that obtained in } \\
\text { conventional operation. } \\
\times \text { Additional costs for } \\
\text { the treatment of the } \\
\text { sludge. } \\
\times \text { Generally, pH } \\
\text { adjustment is required, } \\
\text { thus it is not completely } \\
\text { free of chemical } \\
\text { additives. } \\
\times \text { High OPEX. } \\
\times \text { Relatively ineffective } \\
\text { in the elimination of } \\
\text { stable, persistent } \\
\text { organic compounds. }\end{array}$ & Emerging & $\begin{array}{l}\text { Agrochemical } \\
\text { Chemical } \\
\text { Electronics } \\
\text { Petroleum } \\
\text { Textile }\end{array}$ & $\begin{array}{l}\text { (Bhandari \& } \\
\text { Ranade, } \\
\text { 2014; Goh et } \\
\text { al., 2022; } \\
\text { Nidheesh et } \\
\text { al., 2021; } \\
\text { Ramírez } \\
\text { Calderón et } \\
\text { al., 2020; } \\
\text { Simon et al., } \\
\text { 2018; } \\
\text { Tahreen et al., } \\
\text { 2020; Varjani } \\
\text { et al., 2020; L. } \\
\text { Yang et al., } \\
\text { 2021) }\end{array}$ \\
\hline
\end{tabular}




\begin{tabular}{|c|c|c|c|c|}
\hline Technology & $\begin{array}{l}\text { Advantages }(\sqrt{ }) \\
\text { Disadvantages }(x) \text {; } \\
\text { Gaps/Potential to } \\
\text { exploit }(\cdot) .\end{array}$ & Category & $\begin{array}{l}\text { Common or Potencial } \\
\text { Industry Sector }\end{array}$ & Source \\
\hline & $\begin{array}{l}\text { - Development of better } \\
\text { electrodes. }\end{array}$ & & & \\
\hline & $\begin{array}{l}\text { - Development of newer } \\
\text { electrochemical reactor } \\
\text { configurations. }\end{array}$ & & & \\
\hline & $\begin{array}{l}\text { - Improvements in the } \\
\text { kinetic reactions. }\end{array}$ & & & \\
\hline & $\begin{array}{l}\text { - Reduce energy } \\
\text { consumption. }\end{array}$ & & & \\
\hline & $\begin{array}{l}\text { - In IWW treatment } \\
\text { processes, the long- } \\
\text { term reliability has not } \\
\text { completely been } \\
\text { proven. }\end{array}$ & & & \\
\hline & $\begin{array}{l}\text { - Development of new } \\
\text { electrodes material. }\end{array}$ & & & \\
\hline & $\begin{array}{l}\text { - Large-scale } \\
\text { development. }\end{array}$ & & & \\
\hline & $\begin{array}{l}\text { - Engineered } \\
\text { electrochemical } \\
\text { recovery equipment } \\
\text { requires to be more } \\
\text { developed. }\end{array}$ & & & \\
\hline & $\begin{array}{l}\text { - Develop electrodes } \\
\text { materials with high- } \\
\text { density active sites and } \\
\text { high selectivity. }\end{array}$ & & & \\
\hline & $\begin{array}{l}\text { - Integration with } \\
\text { renewable energy } \\
\text { sources. }\end{array}$ & & & \\
\hline \multirow{6}{*}{$\begin{array}{l}\text { Electroflotation } \\
(\mathrm{EF})\end{array}$} & $\begin{array}{l}\sqrt{ } \text { High selectivity of } \\
\text { metals. }\end{array}$ & \multirow[t]{6}{*}{ Emerging } & Battery & \multirow{6}{*}{$\begin{array}{l}\text { (Mohtashami } \\
\text { \& Shang, } \\
\text { 2019; Varjani } \\
\text { et al., 2020; L } \\
\text { Yang et al., } \\
\text { 2021) }\end{array}$} \\
\hline & \multirow{2}{*}{$\begin{array}{l}\sqrt{ } \text { Extensive large-scale } \\
\text { experience. }\end{array}$} & & Electronic & \\
\hline & & & Petroleum & \\
\hline & $\sqrt{ }$ High removal rate. & & Tannery & \\
\hline & $\begin{array}{l}\sqrt{ } \text { More concentrated } \\
\text { sludge. }\end{array}$ & & & \\
\hline & $\begin{array}{l}\sqrt{ } \text { Elimination of certain } \\
\text { volatile pollutants. }\end{array}$ & & & \\
\hline
\end{tabular}




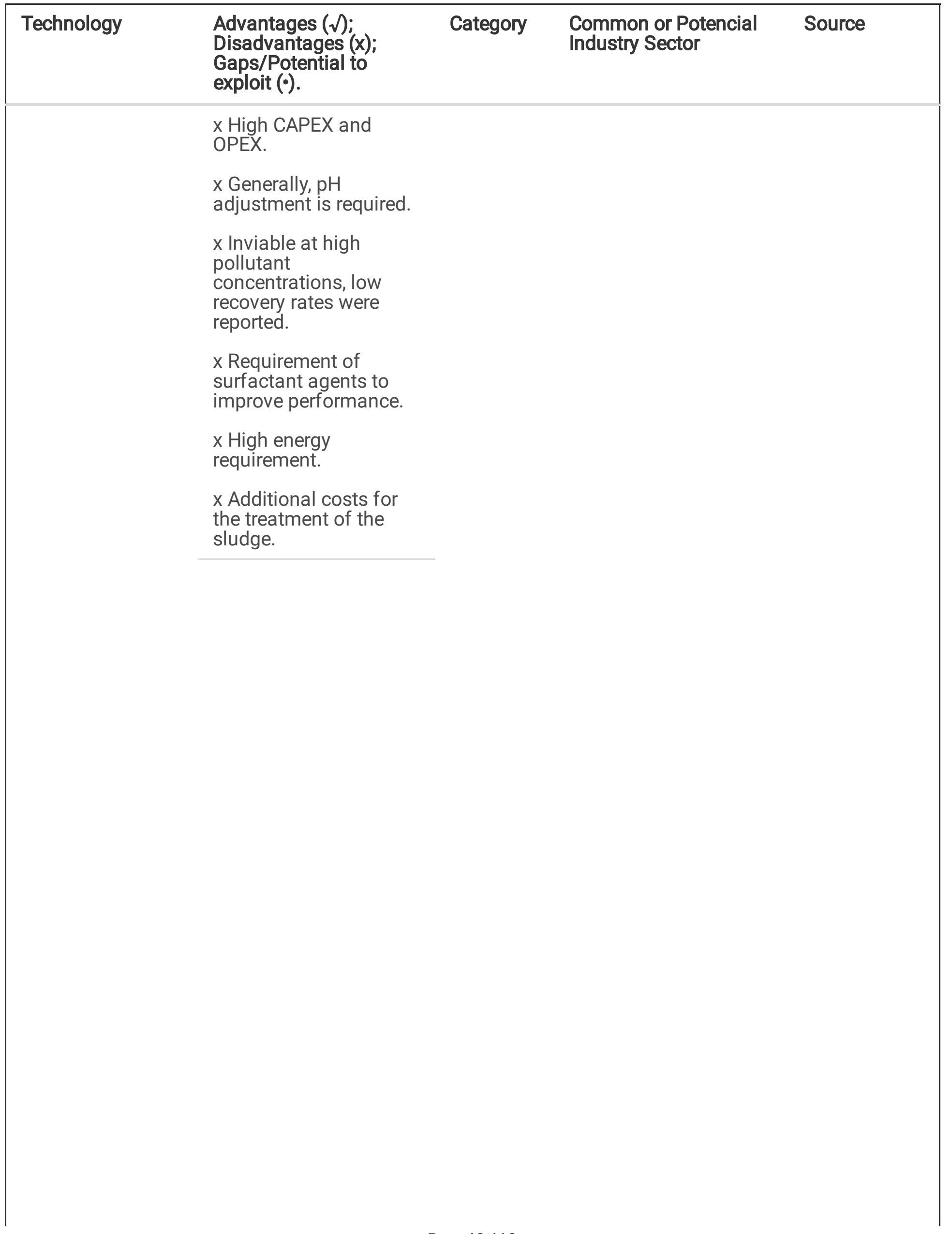




\begin{tabular}{|c|c|c|c|c|}
\hline \multirow[t]{10}{*}{ Technology } & $\begin{array}{l}\text { Advantages }(\sqrt{ }) \text {; } \\
\text { Disadvantages }(x) \text {; } \\
\text { Gaps/Potential to } \\
\text { exploit }(\bullet) \text {. }\end{array}$ & Category & $\begin{array}{l}\text { Common or Potencial } \\
\text { Industry Sector }\end{array}$ & Source \\
\hline & $\begin{array}{l}\text { - In IWW treatment } \\
\text { processes, the long- } \\
\text { term reliability has not } \\
\text { completely been } \\
\text { proven. }\end{array}$ & & & \\
\hline & $\begin{array}{l}\text { - Structure } \\
\text { optimization. }\end{array}$ & & & \\
\hline & $\begin{array}{l}\text { - Reduce energy } \\
\text { consumption. }\end{array}$ & & & \\
\hline & $\begin{array}{l}\text { - Development of new } \\
\text { electrodes material. }\end{array}$ & & & \\
\hline & $\begin{array}{l}\text { - Improvements in the } \\
\text { kinetic of } \\
\text { electroflotation. }\end{array}$ & & & \\
\hline & $\begin{array}{l}\text { - Large-scale } \\
\text { development. }\end{array}$ & & & \\
\hline & $\begin{array}{l}\text { - Engineered } \\
\text { electrochemical } \\
\text { recovery equipment } \\
\text { requires to be more } \\
\text { developed. }\end{array}$ & & & \\
\hline & $\begin{array}{l}\text { - Develop electrodes } \\
\text { materials with high- } \\
\text { density active sites and } \\
\text { high selectivity. }\end{array}$ & & & \\
\hline & $\begin{array}{l}\text { Integration with } \\
\text { renewable energy } \\
\text { sources. }\end{array}$ & & & \\
\hline \multirow{5}{*}{$\begin{array}{l}\text { Electrochemical } \\
\text { precipitation (EP) }\end{array}$} & $\sqrt{ }$ Extensive large-scale & Emerging & Battery & \multirow{5}{*}{$\begin{array}{l}\text { (Muddemann } \\
\text { et al., 2019; } \\
\text { Roy \& } \\
\text { Bhattacharya, } \\
\text { 2014; L. Yang } \\
\text { et al., 2021) }\end{array}$} \\
\hline & & & Chemical & \\
\hline & $\sqrt{ }$ simple operation. & & Electronic & \\
\hline & $\begin{array}{l}\sqrt{\text { High recovery }} \\
\text { (Depends on the } \\
\text { species). }\end{array}$ & & & \\
\hline & $\begin{array}{l}\sqrt{ } \text { Selective recovery } \\
\text { (depends on the } \\
\text { species). }\end{array}$ & & & \\
\hline
\end{tabular}




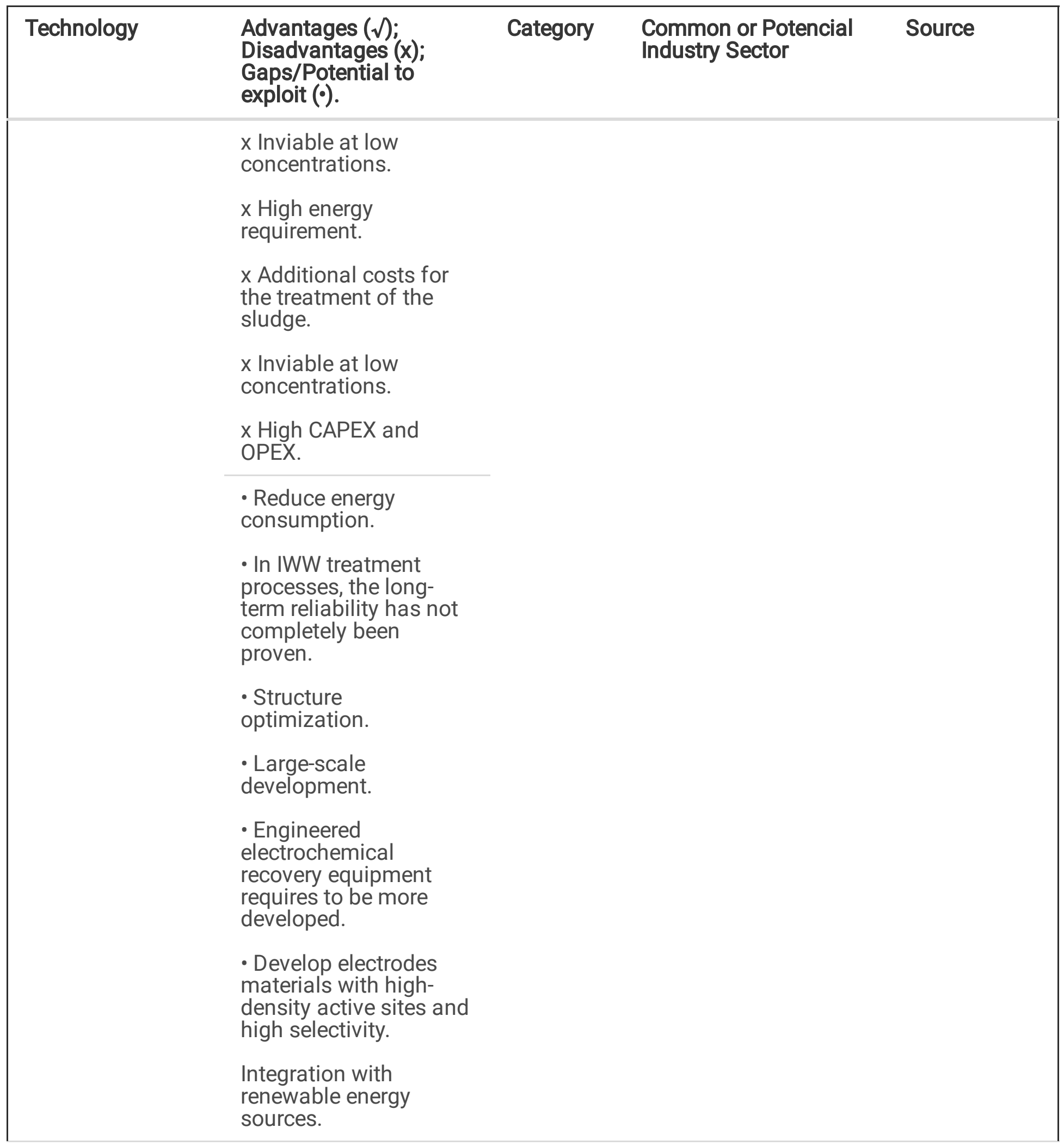




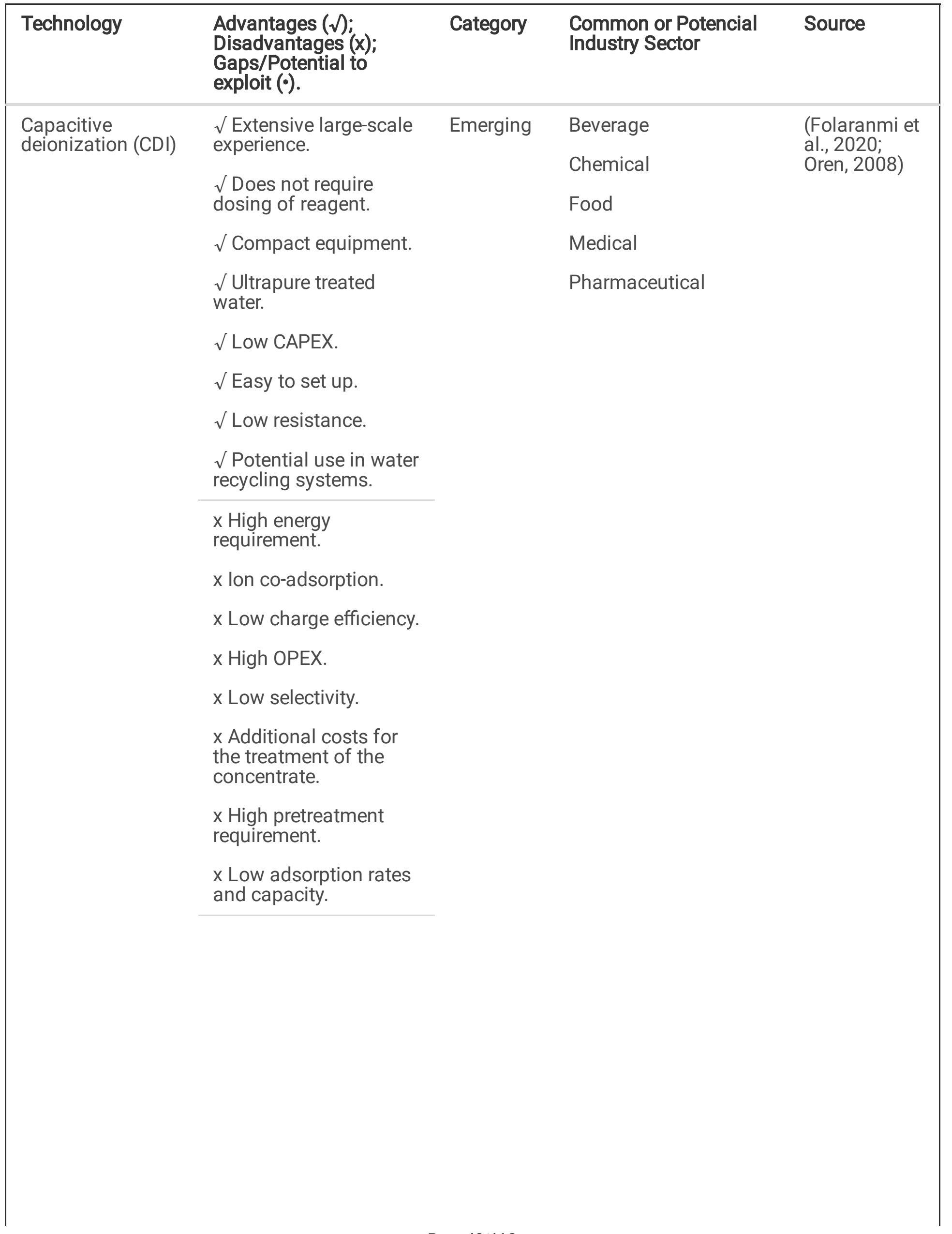




\begin{tabular}{|c|c|c|c|c|}
\hline \multirow[t]{7}{*}{ Technology } & $\begin{array}{l}\text { Advantages }(\sqrt{ }) \text {; } \\
\text { Disadvantages }(x) \text {; } \\
\text { Gaps/Potential to } \\
\text { exploit }(\bullet) \text {. }\end{array}$ & Category & $\begin{array}{l}\text { Common or Potencial } \\
\text { Industry Sector }\end{array}$ & Source \\
\hline & $\begin{array}{l}\text { - Reduce energy } \\
\text { consumption. }\end{array}$ & & & \\
\hline & $\begin{array}{l}\text { - In IWW treatment } \\
\text { processes, the long- } \\
\text { term reliability has not } \\
\text { completely been } \\
\text { proven. }\end{array}$ & & & \\
\hline & $\begin{array}{l}\text { - Develop electrodes } \\
\text { materials with high- } \\
\text { density active sites and } \\
\text { high selectivity. }\end{array}$ & & & \\
\hline & $\begin{array}{l}\text { - Non-Faradaic } \\
\text { operation conditions. }\end{array}$ & & & \\
\hline & $\begin{array}{l}\text { - Reduce energy } \\
\text { consumption. }\end{array}$ & & & \\
\hline & - Hybrid processes. & & & \\
\hline \multirow[t]{10}{*}{ Cavitation } & $\sqrt{ }$ Simple scalability. & Emerging & Fracking & (Bhandari \& \\
\hline & $\sqrt{ }$ High energy & & Medical & 2014; Das et \\
\hline & с & & Petroleum & \\
\hline & $\begin{array}{l}\sqrt{ } \text { Minimized toxic } \\
\text { solvent usage. }\end{array}$ & & & \\
\hline & $\begin{array}{l}\sqrt{ } \text { Simple reactor } \\
\text { design. }\end{array}$ & & & \\
\hline & $\begin{array}{l}\sqrt{ } \text { Zero sludge } \\
\text { production. }\end{array}$ & & & \\
\hline & $\begin{array}{l}\sqrt{ } \text { Enhance chemical } \\
\text { reactions such as AO. }\end{array}$ & & & \\
\hline & $\begin{array}{l}x \text { High CAPEX and } \\
\text { OPEX due to its age. }\end{array}$ & & & \\
\hline & $\begin{array}{l}\text { x Additional chemicals } \\
\text { and oxidants may be } \\
\text { required. }\end{array}$ & & & \\
\hline & $\begin{array}{l}x \text { Cyclic stress on the } \\
\text { system surface causes } \\
\text { fatigue on equipment. }\end{array}$ & & & \\
\hline
\end{tabular}




\begin{tabular}{|c|c|c|c|c|}
\hline Technology & $\begin{array}{l}\text { Advantages }(\sqrt{ }) \\
\text { Disadvantages }(x) \text {; } \\
\text { Gaps/Potential to } \\
\text { exploit }(\cdot) \text {. }\end{array}$ & Category & $\begin{array}{l}\text { Common or Potencial } \\
\text { Industry Sector }\end{array}$ & Source \\
\hline & $\begin{array}{l}\text { - New processes and } \\
\text { equipment designs. }\end{array}$ & & & \\
\hline & $\begin{array}{l}\text { - Principles of } \\
\text { cavitation are still not } \\
\text { clearly understood. }\end{array}$ & & & \\
\hline & $\begin{array}{l}\text { - In IWW treatment } \\
\text { processes, the long- } \\
\text { term reliability has not } \\
\text { completely been } \\
\text { proven. }\end{array}$ & & & \\
\hline & $\begin{array}{l}\text { - Computational fluid } \\
\text { dynamics for better } \\
\text { understanding of flows }\end{array}$ & & & \\
\hline & $\begin{array}{l}\text { - Better theoretical } \\
\text { models for prediction } \\
\text { of process performance } \\
\text { and evaluation. }\end{array}$ & & & \\
\hline & $\begin{array}{l}\text { - Hybrid processes and } \\
\text { process intensification } \\
\text { that result in enhanced } \\
\text { efficiency and efficacy. }\end{array}$ & & & \\
\hline & $\begin{array}{l}\text { - Low cost of operation } \\
\text { and energy. }\end{array}$ & & & \\
\hline \multirow{11}{*}{$\begin{array}{l}\text { Microfiltration } \\
\text { (MF) y } \\
\text { Ultrafiltration (UF) }\end{array}$} & $\begin{array}{l}\sqrt{ } \text { Extensive large-scale } \\
\text { experience. }\end{array}$ & \multirow[t]{11}{*}{ Innovative } & Distillery & \multirow{11}{*}{$\begin{array}{l}\text { (Cui et al., } \\
\text { 2010; } \\
\text { Eykamp, } \\
\text { 1995; Goh et } \\
\text { al., 2022; } \\
\text { Nunes et al., } \\
\text { 2020; } \\
\text { Rezende } \\
\text { Moreira et al., } \\
\text { 2022; Varjani } \\
\text { et al., 2020) }\end{array}$} \\
\hline & \multirow{5}{*}{$\begin{array}{l}\sqrt{ } \text { Simple operation. } \\
\sqrt{ } \text { Easily scalable } \\
\text { modular equipment. } \\
\sqrt{ } \text { Relatively low energy } \\
\text { requirement. }\end{array}$} & & Electronics & \\
\hline & & & Fracking & \\
\hline & & & Medical & \\
\hline & & & Mining & \\
\hline & & & Petroleum & \\
\hline & $\begin{array}{l}\sqrt{ } \text { No sludge is } \\
\text { produced. }\end{array}$ & & Pharmaceutical & \\
\hline & $\begin{array}{l}\sqrt{ } \text { Relatively low CAPEX } \\
\text { and OPEX. }\end{array}$ & & Textile & \\
\hline & $\begin{array}{l}\sqrt{ } \text { Low or no impact on } \\
\text { water chemistry. }\end{array}$ & & & \\
\hline & $\sqrt{ }$ Compact equipment. & & & \\
\hline & $\begin{array}{l}\sqrt{ } \text { Potential use in water } \\
\text { recovery and recycling } \\
\text { systems. }\end{array}$ & & & \\
\hline
\end{tabular}




\begin{tabular}{|c|c|c|c|c|}
\hline Technology & $\begin{array}{l}\text { Advantages }(\sqrt{ }) \\
\text { Disadvantages }(\mathbf{x}) \text {; } \\
\text { Gaps/Potential to } \\
\text { exploit }(\bullet) \text {. }\end{array}$ & Category & $\begin{array}{l}\text { Common or Potencial } \\
\text { Industry Sector }\end{array}$ & Source \\
\hline & $\begin{array}{l}x \text { Additional costs for } \\
\text { the treatment of the } \\
\text { concentrate. }\end{array}$ & & & \\
\hline & $\begin{array}{l}x \text { Limited to certain } \\
\text { particle size. }\end{array}$ & & & \\
\hline & x Fouling. & & & \\
\hline & $\begin{array}{l}\text { - Reduced energy } \\
\text { consumption. }\end{array}$ & & & \\
\hline & - Increased recovery. & & & \\
\hline & $\begin{array}{l}\text { - Improved fouling } \\
\text { resistance }\end{array}$ & & & \\
\hline & $\begin{array}{l}\text { - Development of new } \\
\text { pretreatment and } \\
\text { cleaning strategies. }\end{array}$ & & & \\
\hline & $\begin{array}{l}\text { - Membranes with } \\
\text { higher selectivity, } \\
\text { chemical resistance, } \\
\text { and sufficient } \\
\text { permeance. }\end{array}$ & & & \\
\hline & $\begin{array}{l}\text { - More sustainable } \\
\text { manufacturing } \\
\text { membranes (renewable } \\
\text { raw materials, less } \\
\text { toxic, green solvents, or } \\
\text { no solvents). }\end{array}$ & & & \\
\hline & $\begin{array}{l}\text { - Integration with } \\
\text { renewable energy } \\
\text { sources. }\end{array}$ & & & \\
\hline
\end{tabular}




\begin{tabular}{|c|c|c|c|c|}
\hline Technology & $\begin{array}{l}\text { Advantages }(\sqrt{ }) \text {; } \\
\text { Disadvantages }(x) \text {; } \\
\text { Gaps/Potential to } \\
\text { exploit }(\cdot) \text {. }\end{array}$ & Category & $\begin{array}{l}\text { Common or Potencial } \\
\text { Industry Sector }\end{array}$ & Source \\
\hline Nanofiltration (NF) & $\begin{array}{l}\sqrt{ } \text { Extensive large-scale } \\
\text { experience. } \\
\sqrt{ } \text { Generally, no } \\
\text { additives or other } \\
\text { chemicals are required. } \\
\sqrt{ } \text { No sludge is } \\
\text { produced. } \\
\sqrt{ } \text { Compact equipment. } \\
\sqrt{ } \text { The selective } \\
\text { separation between } \\
\text { monovalent and } \\
\text { multivalent ions is } \\
\text { possible. } \\
\sqrt{ } \text { Easily scalable } \\
\text { modular equipment. } \\
\sqrt{ } \text { Potential use in water } \\
\text { recovery and recycling } \\
\text { systems. } \\
x \text { Relatively high } \\
\text { CAPEX. } \\
x \text { Low remotion of } \\
\text { monovalent salts. } \\
x \text { High pre-treatment } \\
\text { requirement. } \\
x \text { Additional costs for } \\
\text { the treatment of the } \\
\text { concentrate. } \\
x \text { Fouling. }\end{array}$ & Innovative & $\begin{array}{l}\text { Medical } \\
\text { Mining } \\
\text { Nuclear } \\
\text { Petroleum } \\
\text { Pharmaceutical } \\
\text { Textile }\end{array}$ & $\begin{array}{l}\text { (Accepta, } \\
\text { 1997; Cui et } \\
\text { al., 2010; Goh } \\
\text { et al., 2022; } \\
\text { Helmreich \& } \\
\text { Metzger, } \\
\text { 2017; Nunes } \\
\text { et al., 2020; } \\
\text { Rezende } \\
\text { Moreira et al., } \\
\text { 2022; Varjani } \\
\text { et al., 2020) }\end{array}$ \\
\hline
\end{tabular}




\begin{tabular}{|c|c|c|c|c|}
\hline Technology & $\begin{array}{l}\text { Advantages }(\sqrt{ }) \\
\text { Disadvantages }(x) \text {; } \\
\text { Gaps/Potential to } \\
\text { exploit }(\cdot) \text {. }\end{array}$ & Category & $\begin{array}{l}\text { Common or Potencial } \\
\text { Industry Sector }\end{array}$ & Source \\
\hline & $\begin{array}{l}\text { - Reduced energy } \\
\text { consumption. } \\
\text { • Increased recovery. } \\
\text { • Temperature } \\
\text { resistance. } \\
\text { - Narrow pore size } \\
\text { distribution. } \\
\text { - Lower cost } \\
\text { membranes. } \\
\text { - Improved fouling } \\
\text { resistance } \\
\text { - Development of new } \\
\text { pretreatment and } \\
\text { cleaning strategies. } \\
\text { - Higher selectivity } \\
\text { (monovalent vs } \\
\text { divalent selectivity). } \\
\text { - Membranes with } \\
\text { higher chemical } \\
\text { resistance and } \\
\text { sufficient permeance. } \\
\text { - More sustainable } \\
\text { manufacturing } \\
\text { membranes. } \\
\text { - In IWW treatment } \\
\text { processes, the long- } \\
\text { term reliability has not } \\
\text { completely been } \\
\text { proven. } \\
\text { - Integration with } \\
\text { renewable energy } \\
\text { sources. }\end{array}$ & & & \\
\hline
\end{tabular}




\begin{tabular}{|c|c|c|c|c|}
\hline Technology & $\begin{array}{l}\text { Advantages }(\sqrt{ }) \\
\text { Disadvantages }(x) \text {; } \\
\text { Gaps/Potential to } \\
\text { exploit }(\cdot) \text {. }\end{array}$ & Category & $\begin{array}{l}\text { Common or Potencial } \\
\text { Industry Sector }\end{array}$ & Source \\
\hline $\begin{array}{l}\text { Reverse Osmosis } \\
\text { (RO) }\end{array}$ & $\begin{array}{l}\sqrt{ } \text { Extensive large-scale } \\
\text { experience. } \\
\sqrt{ } \text { Generally, no } \\
\text { additives or other } \\
\text { chemicals are required. } \\
\sqrt{ } \text { No sludge is } \\
\text { produced. } \\
\sqrt{ } \text { Compact equipment. } \\
\sqrt{ } \text { Easily scalable } \\
\text { modular equipment. } \\
\sqrt{ } \text { Remotion of all types } \\
\text { of dissolved salts. } \\
\sqrt{ } \text { Potential use in water } \\
\text { recovery and recycling } \\
\text { systems. } \\
\text { x Relatively high OPEX. } \\
\text { x High pre-treatment } \\
\text { requirement. } \\
x \text { Additional costs for } \\
\text { the treatment of the } \\
\text { concentrate. } \\
\text { x The selective } \\
\text { separation between } \\
\text { monovalent and } \\
\text { multivalent ions is not } \\
\text { possible. } \\
x \text { Fouling. }\end{array}$ & Innovative & $\begin{array}{l}\text { Battery } \\
\text { Beverage } \\
\text { Chemical } \\
\text { Distillery } \\
\text { Electronics } \\
\text { Food } \\
\text { Medical } \\
\text { Mining } \\
\text { Nuclear } \\
\text { Petroleum } \\
\text { Pharmaceutical } \\
\text { Textile }\end{array}$ & $\begin{array}{l}\text { (Accepta, } \\
\text { 1997; Cui et } \\
\text { al., 2010; Goh } \\
\text { et al., 2022; } \\
\text { Helmreich \& } \\
\text { Metzger, } \\
\text { 2017; Nunes } \\
\text { et al., 2020; } \\
\text { Rezende } \\
\text { Moreira et al., } \\
\text { 2022; Varjani } \\
\text { et al., 2020; } \\
\text { G. Yadav et } \\
\text { al., 2021) }\end{array}$ \\
\hline
\end{tabular}




\begin{tabular}{|c|c|c|c|c|}
\hline Technology & $\begin{array}{l}\text { Advantages }(\sqrt{ }) ; \\
\text { Disadvantages }(\mathbf{x}) \text {; } \\
\text { Gaps/Potential to } \\
\text { exploit }(\bullet) \text {. }\end{array}$ & Category & $\begin{array}{l}\text { Common or Potencial } \\
\text { Industry Sector }\end{array}$ & Source \\
\hline & $\begin{array}{l}\text { - Reduced energy } \\
\text { consumption. }\end{array}$ & & & \\
\hline & - Increased recovery. & & & \\
\hline & $\begin{array}{l}\text { - Temperature } \\
\text { resistance. }\end{array}$ & & & \\
\hline & $\begin{array}{l}\text { - Improved fouling } \\
\text { resistance }\end{array}$ & & & \\
\hline & $\begin{array}{l}\text { - Development of new } \\
\text { pretreatment and } \\
\text { cleaning strategies. }\end{array}$ & & & \\
\hline & $\begin{array}{l}\text { - Membranes with } \\
\text { chemical resistance } \\
\text { and sufficient } \\
\text { permeance. }\end{array}$ & & & \\
\hline & $\begin{array}{l}\text { - More sustainable } \\
\text { manufacturing } \\
\text { membranes. }\end{array}$ & & & \\
\hline & $\begin{array}{l}\text { - In IWW treatment } \\
\text { processes, the long- } \\
\text { term reliability has not } \\
\text { completely been } \\
\text { proven. }\end{array}$ & & & \\
\hline & $\begin{array}{l}\text { - Integration with } \\
\text { renewable energy } \\
\text { sources. }\end{array}$ & & & \\
\hline
\end{tabular}




\begin{tabular}{|c|c|c|c|c|}
\hline Technology & $\begin{array}{l}\text { Advantages }(\sqrt{ }) \\
\text { Disadvantages }(x) \text {; } \\
\text { Gaps/Potential to } \\
\text { exploit }(\cdot) \text {. }\end{array}$ & Category & $\begin{array}{l}\text { Common or Potencial } \\
\text { Industry Sector }\end{array}$ & Source \\
\hline $\begin{array}{l}\text { Membrane } \\
\text { Distillation (MD) }\end{array}$ & $\begin{array}{l}\sqrt{ } \text { High recovery } \\
\text { potential. } \\
\sqrt{ } \text { Generally, no } \\
\text { additives or other } \\
\text { chemicals are required. } \\
\sqrt{ } \text { No sludge is } \\
\text { produced. } \\
\sqrt{ } \text { Very compact } \\
\text { equipment. } \\
\sqrt{ } \text { Low operating } \\
\text { pressures requirement. } \\
\sqrt{ } \text { Very high } \\
\text { temperatures are not } \\
\text { required (30-60C). } \\
\sqrt{ } \text { Retention of up to } \\
100 \% \text { of non-volatile } \\
\text { substances. } \\
\sqrt{ } \text { Potential use in water } \\
\text { recovery and recycling } \\
\text { systems. } \\
\sqrt{ } \text { Low thermal } \\
\text { conductivity. } \\
\sqrt{ } \text { Can use waste heat. } \\
\sqrt{ } \text { Lower fouling. } \\
\sqrt{ } \text { High resistance to } \\
\text { chemical degradation. }\end{array}$ & Emerging & $\begin{array}{l}\text { Battery } \\
\text { Beverage } \\
\text { Chemical } \\
\text { Food } \\
\text { Fracking } \\
\text { Mining } \\
\text { Nuclear } \\
\text { Textile }\end{array}$ & $\begin{array}{l}\text { (Abdel-Karim } \\
\text { et al., 2021; } \\
\text { Elliott \& Ball, } \\
\text { 2021; Goh et } \\
\text { al., 2022; } \\
\text { Muhammad } \\
\text { Yaqub \& Lee, } \\
\text { 2019; Xie et } \\
\text { al., 2016; A. } \\
\text { Yadav et al., } \\
\text { 2022; Yan et } \\
\text { al., 2018a) }\end{array}$ \\
\hline
\end{tabular}


Gaps/Potential to

exploit ( $)$.

x High pre-treatment requirement.

$x$ Requirement of an extraction solution.

$x$ High CAPEX and OPEX.

$x$ Relatively high energy requirements.

x Unwanted volatile compounds.

$x$ Little or no large-scale experience.

x Fouling.

- Reduced energy consumption.

- Temperature resistance.

- Improved fouling resistance.

- Mechanism of pore wetting.

- Development of new pretreatment and cleaning strategies.

- Membranes with higher selectivity, chemical resistance, and sufficient permeance.

- Prevention of breakthrough.

- Lower cost membranes.

- More sustainable manufacturing membranes.

- Integration with renewable energy sources. 


\begin{tabular}{|c|c|c|c|c|}
\hline \multirow[t]{7}{*}{ Technology } & $\begin{array}{l}\text { Advantages }(\sqrt{ }) \text {; } \\
\text { Disadvantages }(x) \text {; } \\
\text { Gaps/Potential to } \\
\text { exploit }(\cdot) \text {. }\end{array}$ & Category & $\begin{array}{l}\text { Common or Potencial } \\
\text { Industry Sector }\end{array}$ & Source \\
\hline & $\begin{array}{l}\text { - Residual-heat } \\
\text { integration in the } \\
\text { industry. }\end{array}$ & & & \\
\hline & $\begin{array}{l}\text { - In IWW treatment } \\
\text { processes, the long- } \\
\text { term reliability has not } \\
\text { completely been } \\
\text { proven. }\end{array}$ & & & \\
\hline & $\begin{array}{l}\text { - Performance } \\
\text { guarantees for } \\
\text { commercial operations } \\
\text { involving different IWW } \\
\text { and a variety of } \\
\text { pollutants. }\end{array}$ & & & \\
\hline & - Hybrid processes. & & & \\
\hline & $\begin{array}{l}\text { - Draw solution } \\
\text { reconcentration and } \\
\text { recovery process. }\end{array}$ & & & \\
\hline & - Membrane wetting. & & & \\
\hline \multirow{11}{*}{$\begin{array}{l}\text { Forward osmosis } \\
\text { (FO) }\end{array}$} & \multirow{7}{*}{$\begin{array}{l}\sqrt{ } \text { High recovery } \\
\text { potential. } \\
\sqrt{ } \text { Low or no operating } \\
\text { pressures requirement. } \\
\sqrt{ } \text { No sludge is } \\
\text { produced. } \\
\sqrt{ } \text { Compact equipment. } \\
\sqrt{ } \text { Low energy } \\
\text { requirement }\end{array}$} & \multirow[t]{11}{*}{ Emerging } & \multirow{11}{*}{$\begin{array}{l}\text { Agrochemical } \\
\text { Battery } \\
\text { Beverage } \\
\text { Chemical } \\
\text { Food } \\
\text { Textile }\end{array}$} & \multirow{11}{*}{$\begin{array}{l}\text { (Elliott \& Ball, } \\
\text { 2021; Goh et } \\
\text { al., 2022; } \\
\text { Muhammad } \\
\text { Yaqub \& Lee, } \\
\text { 2019; Xie et } \\
\text { al., 2016; Yan } \\
\text { et al., 2018a) }\end{array}$} \\
\hline & & & & \\
\hline & & & & \\
\hline & & & & \\
\hline & & & & \\
\hline & & & & \\
\hline & & & & \\
\hline & $\begin{array}{l}\sqrt{ } \text { High resistance to } \\
\text { Fouling. }\end{array}$ & & & \\
\hline & $\begin{array}{l}\sqrt{ } \text { Potential use in water } \\
\text { recovery and recycling } \\
\text { systems. }\end{array}$ & & & \\
\hline & $\begin{array}{l}\sqrt{ } \text { High rejection rates } \\
\text { of various ions and } \\
\text { contaminants. }\end{array}$ & & & \\
\hline & $\begin{array}{l}\sqrt{ } \text { Can use waste } \\
\text { streams as draw } \\
\text { solutions }\end{array}$ & & & \\
\hline
\end{tabular}




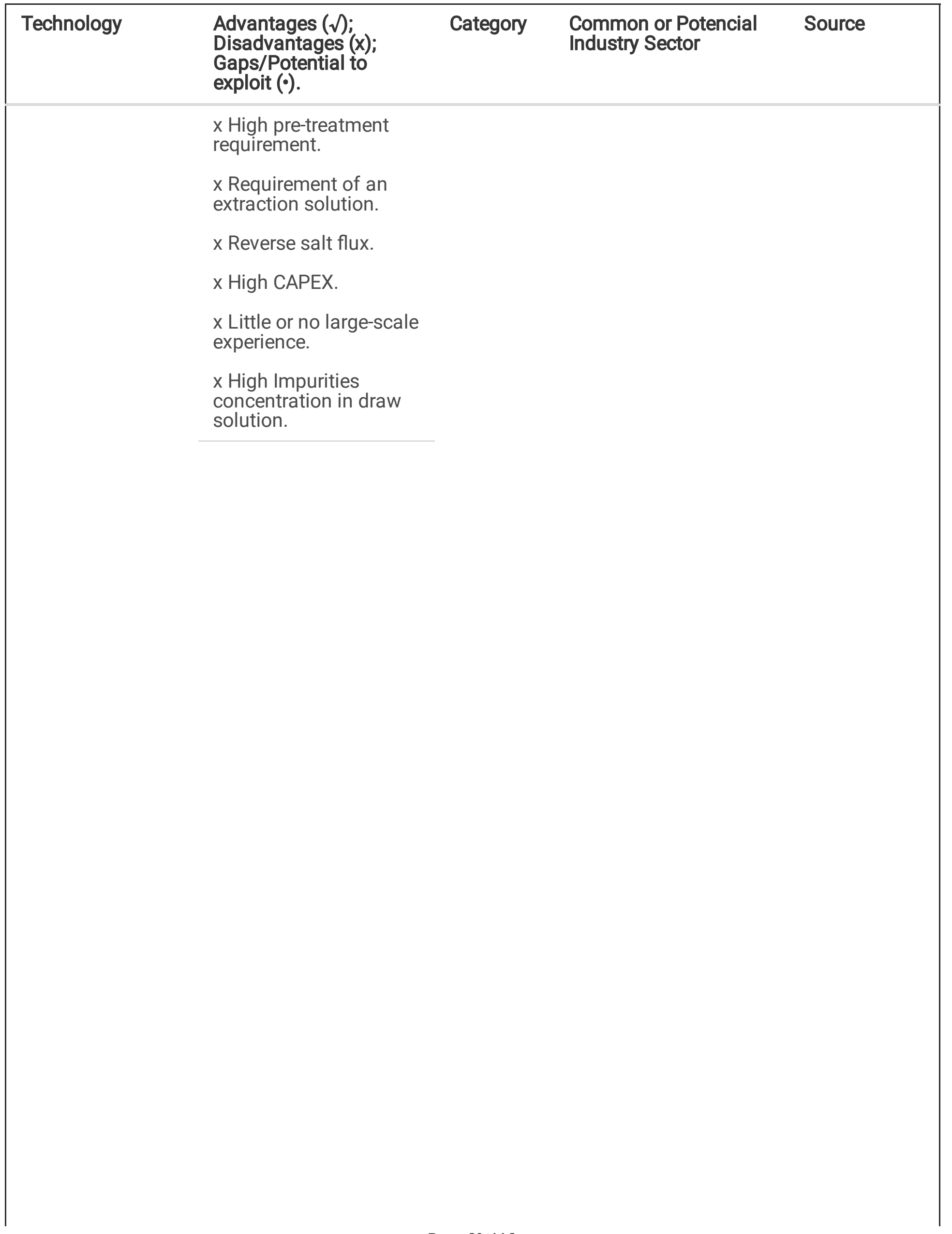




\begin{tabular}{|c|c|c|c|c|}
\hline Technology & $\begin{array}{l}\text { Advantages }(\sqrt{ }) \text {; } \\
\text { Disadvantages }(x) \text {; } \\
\text { Gaps/Potential to } \\
\text { exploit }(\cdot) \text {. }\end{array}$ & Category & $\begin{array}{l}\text { Common or Potencial } \\
\text { Industry Sector }\end{array}$ & Source \\
\hline & - Increased recovery. & & & \\
\hline & - Improved cleanability. & & & \\
\hline & $\begin{array}{l}\text { - Improved fouling } \\
\text { resistance. }\end{array}$ & & & \\
\hline & $\begin{array}{l}\text { - Lower cost } \\
\text { membranes. }\end{array}$ & & & \\
\hline & $\begin{array}{l}\text { - Membranes with } \\
\text { higher selectivity, } \\
\text { chemical resistance, } \\
\text { and sufficient } \\
\text { permeance. }\end{array}$ & & & \\
\hline & - Reverse solute fluxes. & & & \\
\hline & $\begin{array}{l}\text { - Ideal draw solution } \\
\text { and an efficient } \\
\text { recovery system. }\end{array}$ & & & \\
\hline & $\begin{array}{l}\text { - More sustainable } \\
\text { manufacturing } \\
\text { membranes. }\end{array}$ & & & \\
\hline & $\begin{array}{l}\text { - In IWW treatment } \\
\text { processes, the long- } \\
\text { term reliability has not } \\
\text { completely been } \\
\text { proven. }\end{array}$ & & & \\
\hline & $\begin{array}{l}\text { - Performance } \\
\text { guarantees for } \\
\text { commercial operations } \\
\text { involving different IWW } \\
\text { and a variety of } \\
\text { pollutants. }\end{array}$ & & & \\
\hline & $\begin{array}{l}\text { - Draw solution } \\
\text { reconcentration and } \\
\text { recovery process. }\end{array}$ & & & \\
\hline
\end{tabular}




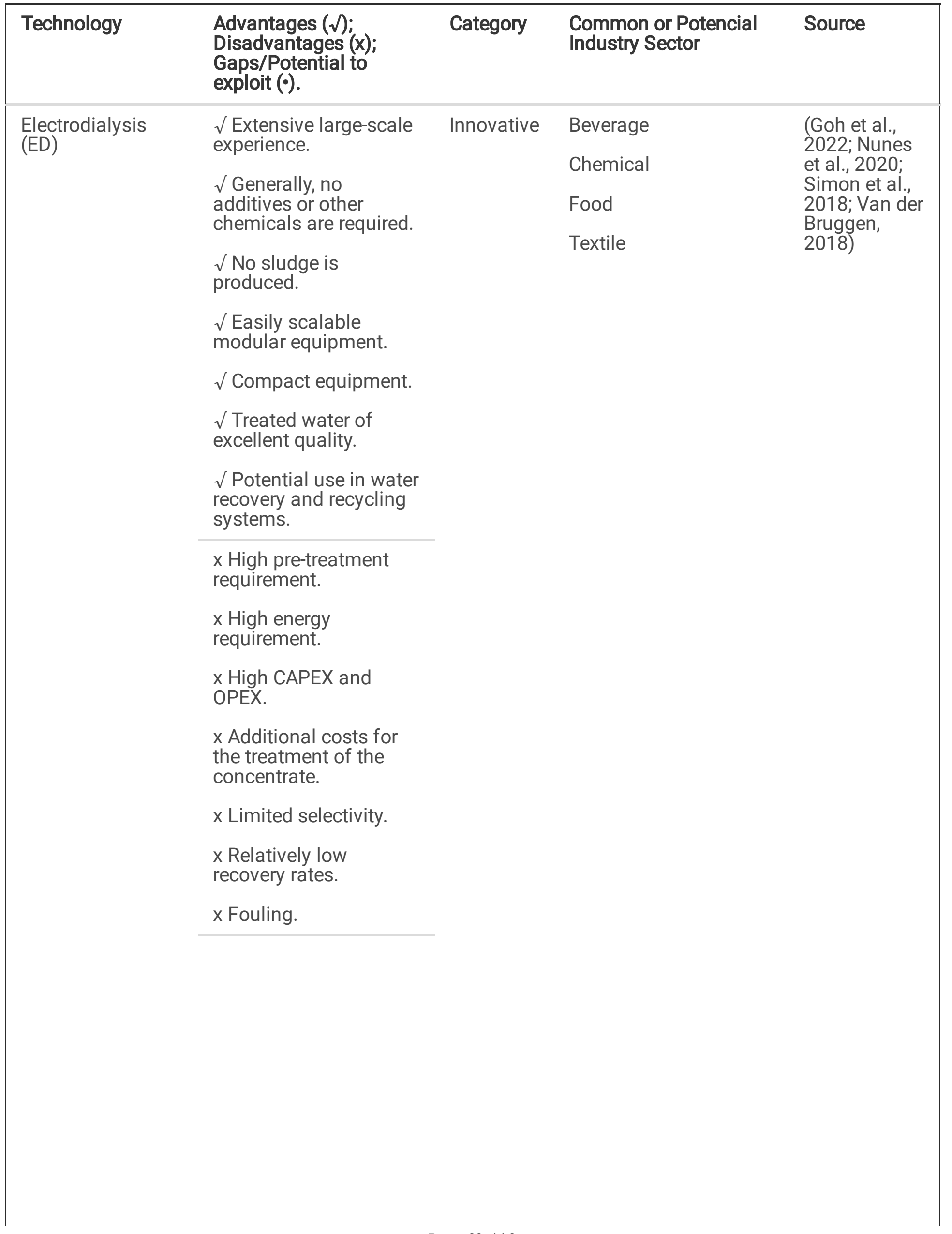




\begin{tabular}{|c|c|c|c|c|}
\hline Technology & $\begin{array}{l}\text { Advantages }(\sqrt{ }) \\
\text { Disadvantages }(x) \text {; } \\
\text { Gaps/Potential to } \\
\text { exploit }(\cdot) \text {. }\end{array}$ & Category & $\begin{array}{l}\text { Common or Potencial } \\
\text { Industry Sector }\end{array}$ & Source \\
\hline & $\begin{array}{l}\text { - Reduced energy } \\
\text { consumption. }\end{array}$ & & & \\
\hline & - Increased recovery. & & & \\
\hline & $\begin{array}{l}\text { - Improved fouling } \\
\text { resistance }\end{array}$ & & & \\
\hline & - Improved cleanability. & & & \\
\hline & $\begin{array}{l}\text { - Membranes with } \\
\text { higher selectivity, } \\
\text { Chemical resistance, } \\
\text { and sufficient } \\
\text { permeance. }\end{array}$ & & & \\
\hline & - Lower resistance. & & & \\
\hline & - Higher selectivity. & & & \\
\hline & $\begin{array}{l}\text { - More sustainable } \\
\text { manufacturing } \\
\text { membranes. }\end{array}$ & & & \\
\hline & $\begin{array}{l}\text { - In IWW treatment } \\
\text { processes, the long- } \\
\text { term reliability has not } \\
\text { completely been } \\
\text { proven. }\end{array}$ & & & \\
\hline & $\begin{array}{l}\text { - Performance } \\
\text { guarantees for } \\
\text { commercial operations } \\
\text { involving different IWW } \\
\text { and a variety of } \\
\text { pollutants. }\end{array}$ & & & \\
\hline & $\begin{array}{l}\text { - Integration with } \\
\text { renewable energy } \\
\text { sources. }\end{array}$ & & & \\
\hline \multirow[t]{6}{*}{$\begin{array}{l}\text { Membrane } \\
\text { electrolysis (ME) }\end{array}$} & $\begin{array}{l}\sqrt{ } \text { No sludge is } \\
\text { produced. }\end{array}$ & \multirow[t]{6}{*}{ Emerging } & Beverage & \multirow{6}{*}{$\begin{array}{l}\text { (Jaroszek \& } \\
\text { Dydo, 2016b; } \\
\text { Paidar et al., } \\
\text { 2016) }\end{array}$} \\
\hline & \multirow{2}{*}{$\begin{array}{l}\sqrt{ } \text { Easily scalable } \\
\text { modular equipment. }\end{array}$} & & Chemical & \\
\hline & & & Food & \\
\hline & $\sqrt{ }$ Compact equipment. & & Medical & \\
\hline & $\begin{array}{l}\sqrt{ } \text { Treated water of } \\
\text { excellent quality. }\end{array}$ & & Pharmaceutical & \\
\hline & $\begin{array}{l}\sqrt{ } \text { Potential use in water } \\
\text { recovery and recycling } \\
\text { systems. }\end{array}$ & & & \\
\hline
\end{tabular}




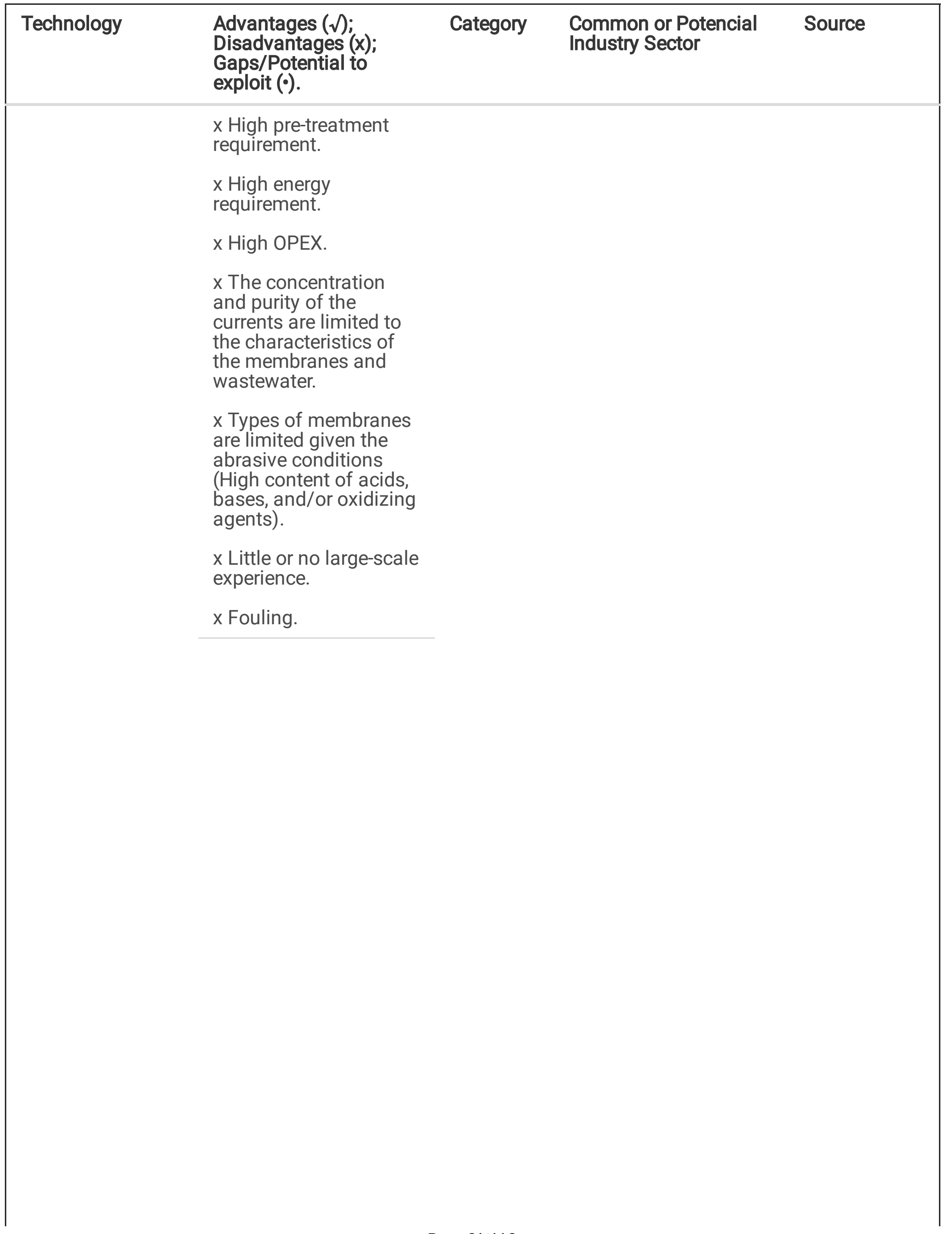




\begin{tabular}{|c|c|c|c|c|}
\hline Technology & $\begin{array}{l}\text { Advantages }(\sqrt{ }) \\
\text { Disadvantages }(x) \text {; } \\
\text { Gaps/Potential to } \\
\text { exploit }(\cdot) \text {. }\end{array}$ & Category & $\begin{array}{l}\text { Common or Potencial } \\
\text { Industry Sector }\end{array}$ & Source \\
\hline & $\begin{array}{l}\text { - Reduced energy } \\
\text { consumption. }\end{array}$ & & & \\
\hline & - Increased recovery. & & & \\
\hline & $\begin{array}{l}\text { - Improved fouling } \\
\text { resistance. }\end{array}$ & & & \\
\hline & $\begin{array}{l}\text { - Lower cost } \\
\text { membranes and } \\
\text { electrodes. }\end{array}$ & & & \\
\hline & - Improved cleanability. & & & \\
\hline & $\begin{array}{l}\text { - Membranes with } \\
\text { higher selectivity, } \\
\text { Chemical resistance, } \\
\text { and sufficient } \\
\text { permeance. }\end{array}$ & & & \\
\hline & $\begin{array}{l}\text { - Higher thermal } \\
\text { stability. }\end{array}$ & & & \\
\hline & $\begin{array}{l}\text { - More sustainable } \\
\text { manufacturing } \\
\text { membranes. }\end{array}$ & & & \\
\hline & $\begin{array}{l}\text { - In IWW treatment } \\
\text { processes, the long- } \\
\text { term reliability has not } \\
\text { completely been } \\
\text { proven. }\end{array}$ & & & \\
\hline & $\begin{array}{l}\text { - Performance } \\
\text { guarantees for } \\
\text { commercial operations } \\
\text { involving different IWW } \\
\text { and a variety of } \\
\text { pollutants. }\end{array}$ & & & \\
\hline & $\begin{array}{l}\text { - Development of } \\
\text { bipolar membranes. }\end{array}$ & & & \\
\hline & $\begin{array}{l}\text { - Temperature } \\
\text { resistance. }\end{array}$ & & & \\
\hline
\end{tabular}




\begin{tabular}{|c|c|c|c|c|}
\hline Technology & $\begin{array}{l}\text { Advantages }(\sqrt{ }) ; \\
\text { Disadvantages }(x) \text {; } \\
\text { Gaps/Potential to } \\
\text { exploit }(\cdot) .\end{array}$ & Category & $\begin{array}{l}\text { Common or Potencial } \\
\text { Industry Sector }\end{array}$ & Source \\
\hline \multirow{15}{*}{$\begin{array}{l}\text { Electro- } \\
\text { Electrodialysis } \\
\text { (EED) }\end{array}$} & \multirow{4}{*}{$\begin{array}{l}\sqrt{ } \text { No sludge is } \\
\text { produced. } \\
\sqrt{ } \text { Compact equipment. } \\
\sqrt{ } \text { Treated water of } \\
\text { excellent quality. }\end{array}$} & \multirow[t]{4}{*}{ Research } & Beverage & \multirow{15}{*}{$\begin{array}{l}\text { (Handojo et } \\
\text { al., 2019; } \\
\text { Jaroszek \& } \\
\text { Dydo, 2016b; } \\
\text { Wei et al., } \\
\text { 2013; Wu et } \\
\text { al., 2019) }\end{array}$} \\
\hline & & & Chemical & \\
\hline & & & Food & \\
\hline & & & Medical & \\
\hline & $\begin{array}{l}\sqrt{ } \text { High purity by- } \\
\text { products. }\end{array}$ & & Pharmaceutica & \\
\hline & $\begin{array}{l}\sqrt{ } \text { Potential use in water } \\
\text { recovery and recycling } \\
\text { systems. }\end{array}$ & & & \\
\hline & $\begin{array}{l}x \text { High pre-treatment } \\
\text { requirement. }\end{array}$ & & & \\
\hline & $\begin{array}{l}\text { x High energy } \\
\text { requirement. }\end{array}$ & & & \\
\hline & x High OPEX. & & & \\
\hline & $\begin{array}{l}x \text { The concentration } \\
\text { and purity of the } \\
\text { currents are limited to } \\
\text { the characteristics of } \\
\text { the membranes and } \\
\text { wastewater. }\end{array}$ & & & \\
\hline & $\begin{array}{l}x \text { Types of membranes } \\
\text { are limited given the } \\
\text { abrasive conditions } \\
\text { (High content of acids, } \\
\text { bases, and/or oxidizing } \\
\text { agents). }\end{array}$ & & & \\
\hline & $\begin{array}{l}x \text { Low concentration in } \\
\text { product solutions. }\end{array}$ & & & \\
\hline & $\begin{array}{l}\text { x Requirement of a pre- } \\
\text { treatment. }\end{array}$ & & & \\
\hline & $\begin{array}{l}\text { x Little or no large-scale } \\
\text { experience. }\end{array}$ & & & \\
\hline & x Fouling. & & & \\
\hline
\end{tabular}




\begin{tabular}{|c|c|c|c|c|}
\hline Technology & $\begin{array}{l}\text { Advantages }(\sqrt{ }) \\
\text { Disadvantages }(x) \text {; } \\
\text { Gaps/Potential to } \\
\text { exploit }(\cdot) .\end{array}$ & Category & $\begin{array}{l}\text { Common or Potencial } \\
\text { Industry Sector }\end{array}$ & Source \\
\hline & $\begin{array}{l}\text { - Reduced energy } \\
\text { consumption. } \\
\text { - Increased recovery. } \\
\text { • Improved fouling } \\
\text { resistance } \\
\text { - Improved cleanability. } \\
\text { - Membranes with } \\
\text { higher selectivity, } \\
\text { chemical resistance, } \\
\text { and sufficient } \\
\text { permeance. } \\
\text { - Lower resistance. } \\
\text { - Higher selectivity } \\
\text { (monovalent vs } \\
\text { divalent selectivity). } \\
\text { - Lower cost } \\
\text { membranes and } \\
\text { electrodes. } \\
\text { - More sustainable } \\
\text { manufacturing } \\
\text { membranes. } \\
\text { - In IWW treatment } \\
\text { processes, the long- } \\
\text { term reliability has not } \\
\text { completely been } \\
\text { proven. } \\
\text { - Performance } \\
\text { guarantees for } \\
\text { commercial operations } \\
\text { involving different IWW } \\
\text { and a variety of } \\
\text { pollutants. }\end{array}$ & & & \\
\hline
\end{tabular}




\begin{tabular}{|c|c|c|c|c|}
\hline Technology & $\begin{array}{l}\text { Advantages }(\sqrt{ }) \\
\text { Disadvantages }(x) \text {; } \\
\text { Gaps/Potential to } \\
\text { exploit }(\cdot) \text {. }\end{array}$ & Category & $\begin{array}{l}\text { Common or Potencial } \\
\text { Industry Sector }\end{array}$ & Source \\
\hline $\begin{array}{l}\text { Bipolar Membrane } \\
\text { Electrodialysis } \\
\text { (BMED) }\end{array}$ & $\begin{array}{l}\sqrt{ } \text { No sludge is } \\
\text { produced. } \\
\sqrt{ } \text { Compact equipment. } \\
\sqrt{ } \text { Treated water of } \\
\text { excellent quality. } \\
\sqrt{ } \text { High purity by- } \\
\text { products. } \\
\sqrt{ } \text { High rate of water } \\
\text { separation. } \\
\sqrt{ } \text { Potential use in water } \\
\text { recovery and recycling } \\
\text { systems. } \\
\text { x High pre-treatment } \\
\text { requirement. } \\
\text { x High energy } \\
\text { requirement. } \\
\text { x High OPEX. } \\
\text { x The concentration } \\
\text { and purity of the } \\
\text { currents are limited to } \\
\text { the characteristics of } \\
\text { the membranes and } \\
\text { wastewater. } \\
\text { x Types of membranes } \\
\text { are limited given the } \\
\text { abrasive conditions } \\
\text { (High content of acids, } \\
\text { bases, and/or oxidizing } \\
\text { agents). } \\
\text { x limited application in } \\
\text { divalent metals. } \\
\times \text { Low concentration in } \\
\text { product solutions. } \\
x \text { Little or no large-scale } \\
\text { experience. } \\
x \text { Fouling. } \\
\text {. }\end{array}$ & Emerging & $\begin{array}{l}\text { Beverage } \\
\text { Chemical } \\
\text { Food } \\
\text { PharmaceuticalMedical }\end{array}$ & $\begin{array}{l}\text { (Nowak et al., } \\
\text { 2014; } \\
\text { Pisarska, } \\
\text { Jaroszek, et } \\
\text { al., 2017; } \\
\text { Pisarska, } \\
\text { Mikołajczak, } \\
\text { et al., 2017; C. } \\
\text { Yang et al., } \\
\text { 2003) }\end{array}$ \\
\hline
\end{tabular}




\begin{tabular}{|c|c|c|c|c|}
\hline Technology & $\begin{array}{l}\text { Advantages }(\sqrt{ }) \\
\text { Disadvantages }(x) \text {; } \\
\text { Gaps/Potential to } \\
\text { exploit }(\cdot) \text {. }\end{array}$ & Category & $\begin{array}{l}\text { Common or Potencial } \\
\text { Industry Sector }\end{array}$ & Source \\
\hline & $\begin{array}{l}\text { - Reduced energy } \\
\text { consumption. }\end{array}$ & & & \\
\hline & - Increased recovery. & & & \\
\hline & $\begin{array}{l}\text { - Improved fouling } \\
\text { resistance. }\end{array}$ & & & \\
\hline & - Improved cleanability. & & & \\
\hline & $\begin{array}{l}\text { - Membranes with } \\
\text { higher selectivity and } \\
\text { sufficient permeance. }\end{array}$ & & & \\
\hline & - Lower resistance. & & & \\
\hline & $\begin{array}{l}\text { - Lower cost } \\
\text { membranes and } \\
\text { electrodes. }\end{array}$ & & & \\
\hline & $\begin{array}{l}\text { - Performance } \\
\text { guarantees for } \\
\text { commercial operations } \\
\text { involving different IWW } \\
\text { and a variety of } \\
\text { pollutants. }\end{array}$ & & & \\
\hline & $\begin{array}{l}\text { - More sustainable } \\
\text { manufacturing } \\
\text { membranes. }\end{array}$ & & & \\
\hline & $\begin{array}{l}\text { - Development of } \\
\text { bipolar membranes. }\end{array}$ & & & \\
\hline & $\begin{array}{l}\text { - In IWW treatment } \\
\text { processes, the long- } \\
\text { term reliability has not } \\
\text { completely been } \\
\text { proven. }\end{array}$ & & & \\
\hline
\end{tabular}




\begin{tabular}{|c|c|c|c|c|}
\hline Technology & $\begin{array}{l}\text { Advantages }(\sqrt{ }) \\
\text { Disadvantages }(x) \text {; } \\
\text { Gaps/Potential to } \\
\text { exploit }(\bullet) \text {. }\end{array}$ & Category & $\begin{array}{l}\text { Common or Potencial } \\
\text { Industry Sector }\end{array}$ & Source \\
\hline \multirow{17}{*}{$\begin{array}{l}\text { Membrane- } \\
\text { assisted electron } \\
\text { ionization (EDI) }\end{array}$} & \multirow{2}{*}{$\begin{array}{l}\sqrt{ } \text { Extensive large-scale } \\
\text { experience. }\end{array}$} & \multirow[t]{5}{*}{ Research } & Beverage & \multirow{17}{*}{$\begin{array}{l}\text { (Handojo et } \\
\text { al., 2019) }\end{array}$} \\
\hline & & & Chemical & \\
\hline & \multirow{2}{*}{$\begin{array}{l}\sqrt{ } \text { Generally, no } \\
\text { additives or other } \\
\text { chemicals are required. }\end{array}$} & & Food & \\
\hline & & & Medical & \\
\hline & $\begin{array}{l}\sqrt{ } \text { No sludge is } \\
\text { produced. }\end{array}$ & & Pharmaceutica & \\
\hline & $\begin{array}{l}\sqrt{ } \text { Easily scalable } \\
\text { modular equipment. }\end{array}$ & & & \\
\hline & $\sqrt{ }$ Compact equipment. & & & \\
\hline & $\begin{array}{l}\sqrt{ } \text { Ultrapure treated } \\
\text { water. }\end{array}$ & & & \\
\hline & $\sqrt{ }$ High recovery rates. & & & \\
\hline & $\begin{array}{l}\sqrt{ } \text { Potential use in water } \\
\text { recovery and recycling } \\
\text { systems. }\end{array}$ & & & \\
\hline & $\begin{array}{l}\text { x High pre-treatment } \\
\text { requirement. }\end{array}$ & & & \\
\hline & $\begin{array}{l}\text { x High energy } \\
\text { requirement. }\end{array}$ & & & \\
\hline & $\begin{array}{l}x \text { High CAPEX and } \\
\text { OPEX. }\end{array}$ & & & \\
\hline & $\begin{array}{l}x \text { Little or no large-scale } \\
\text { experience. }\end{array}$ & & & \\
\hline & $\begin{array}{l}x \text { Additional costs for } \\
\text { the treatment of the } \\
\text { concentrate. }\end{array}$ & & & \\
\hline & $\begin{array}{l}\text { x Limited application in } \\
\text { wastewater. }\end{array}$ & & & \\
\hline & x Fouling. & & & \\
\hline
\end{tabular}




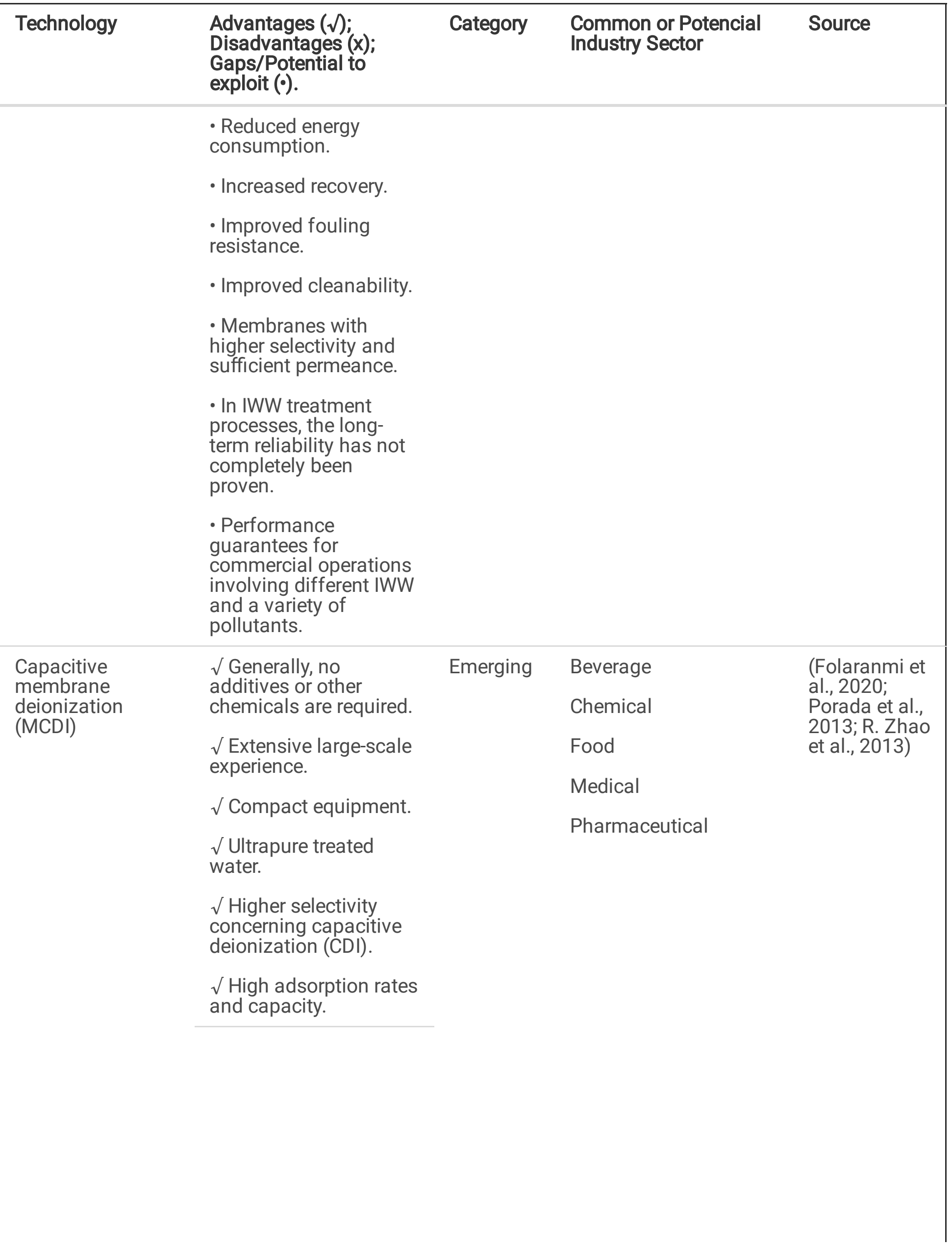




\begin{tabular}{|c|c|c|c|c|}
\hline Technology & $\begin{array}{l}\text { Advantages }(\sqrt{ }) ; \\
\text { Disadvantages }(\mathbf{x}) \text {; } \\
\text { Gaps/Potential to } \\
\text { exploit }(\bullet) \text {. }\end{array}$ & Category & $\begin{array}{l}\text { Common or Potencial } \\
\text { Industry Sector }\end{array}$ & Source \\
\hline & $\begin{array}{l}\text { x High pre-treatment } \\
\text { requirement. }\end{array}$ & & & \\
\hline & x High OPEX. & & & \\
\hline & $\begin{array}{l}x \text { High resistance in the } \\
\text { system. }\end{array}$ & & & \\
\hline & $\begin{array}{l}x \text { Additional costs for } \\
\text { the treatment of the } \\
\text { concentrate. }\end{array}$ & & & \\
\hline & $x\rfloor$ Fouling. & & & \\
\hline & $\begin{array}{l}\text { - Reduced energy } \\
\text { consumption. }\end{array}$ & & & \\
\hline & - Increased recovery. & & & \\
\hline & $\begin{array}{l}\text { - Improved fouling } \\
\text { resistance. }\end{array}$ & & & \\
\hline & - Improved cleanability. & & & \\
\hline & $\begin{array}{l}\text { - Membranes with } \\
\text { higher selectivity and } \\
\text { sufficient permeance. }\end{array}$ & & & \\
\hline & $\begin{array}{l}\text { - In IWW treatment } \\
\text { processes, the long- } \\
\text { term reliability has not } \\
\text { completely been } \\
\text { proven. }\end{array}$ & & & \\
\hline & $\begin{array}{l}\text { - More sustainable } \\
\text { manufacturing } \\
\text { membranes. }\end{array}$ & & & \\
\hline & $\begin{array}{l}\text { - Performance } \\
\text { guarantees for } \\
\text { commercial operations } \\
\text { involving different IWW } \\
\text { and a variety of } \\
\text { pollutants. }\end{array}$ & & & \\
\hline
\end{tabular}


Table 3

Biological treatment units: Advantages, disadvantages and gaps/potential to exploit.

\begin{tabular}{|c|c|c|c|c|}
\hline Technology & $\begin{array}{l}\text { Advantages }(\sqrt{ }) \\
\text { Disadvantages }(x) \text {; } \\
\text { Gaps/Potential to exploit }(\cdot)\end{array}$ & Category & $\begin{array}{l}\text { Common or } \\
\text { Potencial } \\
\text { Industry Sector }\end{array}$ & Source \\
\hline \multirow{13}{*}{$\begin{array}{l}\text { Conventional } \\
\text { activated sludge } \\
\text { (CAS) }\end{array}$} & $\begin{array}{l}\sqrt{ } \text { Simple technology, } \\
\text { economically attractive. }\end{array}$ & \multirow[t]{13}{*}{ Established } & Agriculture & \multirow{13}{*}{$\begin{array}{l}\text { (Bhattacharya } \\
\text { et al., 2017; } \\
\text { Fernández- } \\
\text { alba, 2006; } \\
\text { Ranade \& } \\
\text { Bhandari, } \\
\text { 2014b; } \\
\text { Stanbury et } \\
\text { al., 2016; } \\
\text { Yildiz Sizirici, } \\
\text { 2012) }\end{array}$} \\
\hline & $\sqrt{ }$ Extensive larae-scale & & Beverage & \\
\hline & experience. & & Distillery & \\
\hline & $\sqrt{ }$ High efficiency in the & & Electronics & \\
\hline & organic matter. & & \multirow[t]{9}{*}{ Food } & \\
\hline & x High generation of sludge. & & & \\
\hline & x High energy requirement. & & & \\
\hline & $\begin{array}{l}x \text { It is required to achieve } \\
\text { optimal operating } \\
\text { conditions. }\end{array}$ & & & \\
\hline & x Relatively slow process. & & & \\
\hline & x High CAPEX. & & & \\
\hline & $\begin{array}{l}\text { x Complex degradation } \\
\text { mechanism. }\end{array}$ & & & \\
\hline & $\begin{array}{l}x \text { Generally, post-treatment is } \\
\text { required. }\end{array}$ & & & \\
\hline & $\begin{array}{l}x \text { Additional costs for the } \\
\text { treatment of the sludge. }\end{array}$ & & & \\
\hline
\end{tabular}




\begin{tabular}{|c|c|c|c|c|}
\hline Technology & $\begin{array}{l}\text { Advantages }(\sqrt{ }) ; \\
\text { Disadvantages }(\mathbf{x}) \text {; } \\
\text { Gaps/Potential to exploit ( } \bullet)\end{array}$ & Category & $\begin{array}{l}\text { Common or } \\
\text { Potencial } \\
\text { Industry Sector }\end{array}$ & Source \\
\hline & $\begin{array}{l}\text { - New microorganisms, that } \\
\text { can degrade emerging and } \\
\text { refractory pollutants more } \\
\text { effectively. }\end{array}$ & & & \\
\hline & $\begin{array}{l}\text { - New equipment designs, } \\
\text { especially for increased } \\
\text { effectiveness and less space } \\
\text { and sludge production. }\end{array}$ & & & \\
\hline & $\begin{array}{l}\text { - Hybrid processes and } \\
\text { process intensification that } \\
\text { result in increased efficiency } \\
\text { and efficacy, thus the } \\
\text { implementation of the } 3 R \\
\text { scheme. }\end{array}$ & & & \\
\hline & $\begin{array}{l}\text { - Reduce the cost of } \\
\text { operation and energy } \\
\text { requirement. }\end{array}$ & & & \\
\hline & $\begin{array}{l}\text { - Performance guarantees } \\
\text { for commercial operations } \\
\text { involving different IWW and } \\
\text { a variety of pollutants. }\end{array}$ & & & \\
\hline & $\begin{array}{l}\text { - Properly managed biomass } \\
\text { and exploited toward } \\
\text { recovery and reuse. }\end{array}$ & & & \\
\hline & $\begin{array}{l}\text { - Reduce sludge volume } \\
\text { produced. }\end{array}$ & & & \\
\hline & $\begin{array}{l}\text { - Reuse for the sludge } \\
\text { produced. }\end{array}$ & & & \\
\hline
\end{tabular}




\begin{tabular}{|c|c|c|c|c|}
\hline Technology & $\begin{array}{l}\text { Advantages }(\sqrt{ }) \\
\text { Disadvantages }(\mathbf{x}) \text {; } \\
\text { Gaps/Potential to exploit }(\cdot)\end{array}$ & Category & $\begin{array}{l}\text { Common or } \\
\text { Potencial } \\
\text { Industry Sector }\end{array}$ & Source \\
\hline \multirow{18}{*}{$\begin{array}{l}\text { Aerobic trickling } \\
\text { filter (ATF). }\end{array}$} & \multirow{3}{*}{$\begin{array}{l}\sqrt{ } \text { Relatively simple } \\
\text { technology. } \\
\sqrt{ } \text { Extensive large-scale } \\
\text { experience. }\end{array}$} & \multirow[t]{3}{*}{ Established } & Agriculture & \multirow{18}{*}{$\begin{array}{l}\text { (Moran, 2018; } \\
\text { Stanbury et } \\
\text { al., 2016; } \\
\text { Yildiz Sizirici, } \\
\text { 2012) }\end{array}$} \\
\hline & & & Beverage & \\
\hline & & & Distillery & \\
\hline & $\begin{array}{l}\sqrt{ } \text { High efficiency in the } \\
\text { removal of biodegradable } \\
\text { organic matter. }\end{array}$ & & Food & \\
\hline & $\begin{array}{l}\sqrt{ } \text { Reduces the air injection } \\
\text { requirement. }\end{array}$ & & & \\
\hline & $\begin{array}{l}\sqrt{ } \text { Generally, it does not } \\
\text { require recirculation of } \\
\text { sludge. }\end{array}$ & & & \\
\hline & $\begin{array}{l}\sqrt{ } \text { Biomass concentrations in } \\
\text { the bioreactor are higher } \\
\text { than in conventional } \\
\text { reactors. }\end{array}$ & & & \\
\hline & $\mathrm{x}$ High generation of sludge. & & & \\
\hline & $\begin{array}{l}x \text { The assistance of a settler } \\
\text { is still required, given that the } \\
\text { biomass is capable of } \\
\text { detaching from the support } \\
\text { surfaces. }\end{array}$ & & & \\
\hline & $\begin{array}{l}x \text { High energy requirement } \\
\text { for movement of mechanical } \\
\text { parts. }\end{array}$ & & & \\
\hline & x Risk of system clogging. & & & \\
\hline & $\begin{array}{l}\mathrm{x} \text { It is required to achieve } \\
\text { optimal operating } \\
\text { conditions. }\end{array}$ & & & \\
\hline & x Relatively slow process. & & & \\
\hline & x High CAPEX. & & & \\
\hline & $\begin{array}{l}\text { x Complex degradation } \\
\text { mechanism. }\end{array}$ & & & \\
\hline & $\begin{array}{l}\text { x Complex maintenance, } \\
\text { which implies higher OPEX } \\
\text { cost. }\end{array}$ & & & \\
\hline & $\begin{array}{l}x \text { Generally, post-treatment is } \\
\text { required. }\end{array}$ & & & \\
\hline & $\begin{array}{l}x \text { Additional costs for the } \\
\text { treatment of the sludge. }\end{array}$ & & & \\
\hline
\end{tabular}




\begin{tabular}{|c|c|c|c|c|}
\hline Technology & $\begin{array}{l}\text { Advantages }(\sqrt{ }) ; \\
\text { Disadvantages }(\mathbf{x}) \\
\text { Gaps/Potential to exploit ( }(\cdot)\end{array}$ & Category & $\begin{array}{l}\text { Common or } \\
\text { Potencial } \\
\text { Industry Sector }\end{array}$ & Source \\
\hline & $\begin{array}{l}\text { - Low cost of operation and } \\
\text { energy. }\end{array}$ & & & \\
\hline & $\begin{array}{l}\text { - New microorganisms, that } \\
\text { can degrade emerging and } \\
\text { refractory pollutants more } \\
\text { effectively. }\end{array}$ & & & \\
\hline & $\begin{array}{l}\text { - Hybrid processes and } \\
\text { process intensification that } \\
\text { result in increased efficiency } \\
\text { and efficacy, thus the } \\
\text { implementation of the 3R } \\
\text { scheme. }\end{array}$ & & & \\
\hline & $\begin{array}{l}\text { - Non-complex equipment to } \\
\text { reduce maintenance costs. }\end{array}$ & & & \\
\hline & $\begin{array}{l}\text { - New design to reduce the } \\
\text { necessity of assistance } \\
\text { settler. }\end{array}$ & & & \\
\hline & $\begin{array}{l}\text { - Biomass is properly } \\
\text { managed and exploited } \\
\text { toward recovery and reuse. }\end{array}$ & & & \\
\hline & $\begin{array}{l}\text { - Performance guarantees } \\
\text { for commercial operations. }\end{array}$ & & & \\
\hline & $\begin{array}{l}\text { - Reuse for the sludge } \\
\text { produced. }\end{array}$ & & & \\
\hline
\end{tabular}




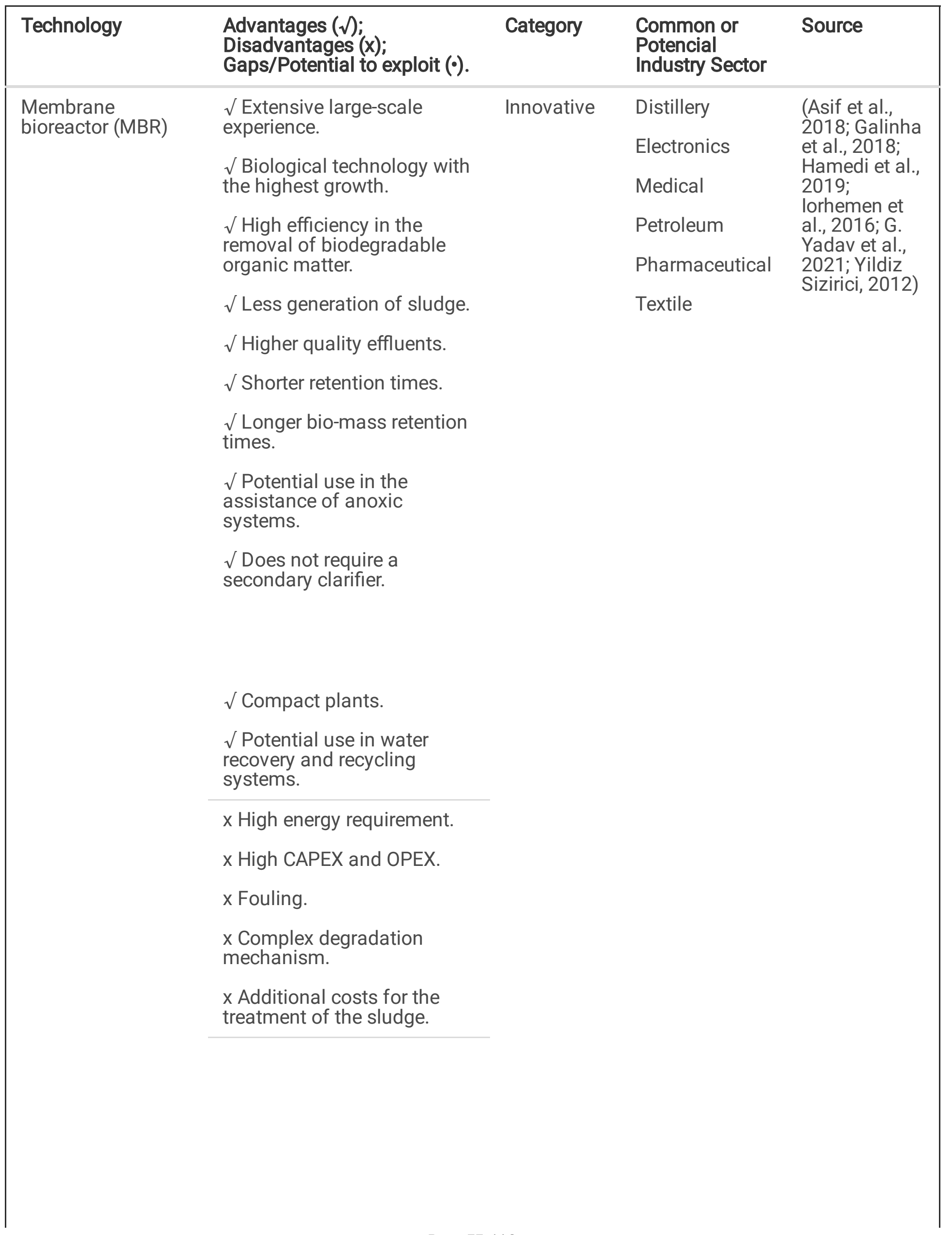




\begin{tabular}{|c|c|c|c|c|}
\hline Technology & $\begin{array}{l}\text { Advantages }(\sqrt{ }) ; \\
\text { Disadvantages }(\mathbf{x}) \text {; } \\
\text { Gaps/Potential to exploit }(\cdot) \text {. }\end{array}$ & Category & $\begin{array}{l}\text { Common or } \\
\text { Potencial } \\
\text { Industry Sector }\end{array}$ & Source \\
\hline & $\begin{array}{l}\text { - Development of low-fouling } \\
\text { membrane systems. }\end{array}$ & & & \\
\hline & $\begin{array}{l}\text { - Investigation of the } \\
\text { characterization and } \\
\text { mechanisms of the fouling } \\
\text { phenomenon. }\end{array}$ & & & \\
\hline & $\begin{array}{l}\text { - New microorganisms, that } \\
\text { can degrade emerging and } \\
\text { refractory pollutants more } \\
\text { effectively. }\end{array}$ & & & \\
\hline & $\begin{array}{l}\text { - Performance guarantees } \\
\text { for commercial operations } \\
\text { involving different IWW and } \\
\text { a variety of pollutants. }\end{array}$ & & & \\
\hline & $\begin{array}{l}\text { - Properly managed biomass } \\
\text { and exploited toward } \\
\text { recovery and reuse. }\end{array}$ & & & \\
\hline & $\begin{array}{l}\text { - Reuse for the sludge } \\
\text { produced. }\end{array}$ & & & \\
\hline & $\begin{array}{l}\text { Integration with renewable } \\
\text { energy sources. }\end{array}$ & & & \\
\hline \multirow[t]{8}{*}{ Biofiltration } & $\sqrt{ }$ Simple design. & Innovative & Agriculture & \multirow{8}{*}{$\begin{array}{l}\text { (Singh et al., } \\
\text { 2019; } \\
\text { Thuptimdang } \\
\text { et al., 2020) }\end{array}$} \\
\hline & $\sqrt{ }$ Low energy requirement. & & Beverage & \\
\hline & $\sqrt{ }$ Non-complex equipment. & & Distillery & \\
\hline & $\sqrt{ }$ Higher treatment potential. & & Food & \\
\hline & $\sqrt{ }$ Low production of sludge. & & & \\
\hline & $\sqrt{ }$ Clogging risk reduction. & & & \\
\hline & $\begin{array}{l}\sqrt{ } \text { Relatively low CAPEX and } \\
\text { OPEX. }\end{array}$ & & & \\
\hline & $\begin{array}{l}\sqrt{ } \text { Potential use in a reuse } \\
\text { water system. }\end{array}$ & & & \\
\hline
\end{tabular}


Advantages $(\sqrt{ })$;

Disadvantages $(x)$;

Category

Gaps/Potential to exploit (•).

$x$ Application has been very

limited to the IWW treatment.

$x$ High sensitivity towards

high salinity, $\mathrm{pH}$, heavy

metals, pesticides, and other pollutants.

$x$ Low efficiency and productivity in IWW treatment.

x It cannot be operated in submerged conditions (Vermifilter).

- Performance guarantees for commercial operations involving different IWW and a variety of pollutants.

- Limited to IWW from the food and beverage industries.

- New equipment designs, especially for increased effectiveness and less space and sludge production.

- Emerging contaminants remotion.

- Understanding of biofilm development and morphological properties.

- Development of novel support material.

- Performance guarantees for commercial operations.

- Hybrid processes and process intensification that result in increased efficiency and efficacy, thus the implementation of the $3 \mathrm{R}$ scheme. 


\begin{tabular}{|c|c|c|c|c|}
\hline Technology & $\begin{array}{l}\text { Advantages }(\sqrt{ }) ; \\
\text { Disadvantages }(x) \text {; } \\
\text { Gaps/Potential to exploit }(\cdot)\end{array}$ & Category & $\begin{array}{l}\text { Common or } \\
\text { Potencial } \\
\text { Industry Sector }\end{array}$ & Source \\
\hline \multirow{18}{*}{$\begin{array}{l}\text { Anaerobic contact } \\
\text { Process (ACP). }\end{array}$} & \multirow{4}{*}{$\begin{array}{l}\sqrt{ } \text { High process efficiency, } \\
\text { even with wastewater with a } \\
\text { high organic load. }\end{array}$} & \multirow[t]{4}{*}{ Established } & Agriculture & \multirow{18}{*}{$\begin{array}{l}\text { (Fernández- } \\
\text { alba, 2006; } \\
\text { Hassan et al., } \\
\text { 2013; Varjani } \\
\text { et al., 2020) }\end{array}$} \\
\hline & & & Beverage & \\
\hline & & & Distillery & \\
\hline & & & Food & \\
\hline & $\begin{array}{l}\sqrt{ } \text { Extensive large-scale } \\
\text { experience. }\end{array}$ & & Petroleum & \\
\hline & $\sqrt{ }$ Low energy consumption. & & & \\
\hline & $\sqrt{ }$ Low production of sludge. & & & \\
\hline & $\sqrt{ }$ Low nutrient requirement. & & & \\
\hline & $\begin{array}{l}\sqrt{ } \text { Obtaining biogas with } \\
\text { potential use in energy } \\
\text { generation. }\end{array}$ & & & \\
\hline & $\sqrt{ }$ Obtaining fertilizers. & & & \\
\hline & $\begin{array}{l}\sqrt{ } \text { Possibility of plant } \\
\text { shutdown without affecting } \\
\text { the system. }\end{array}$ & & & \\
\hline & $\begin{array}{l}x \text { Low effectiveness in } \\
\text { eliminating pathogens and } \\
\text { nutrients. }\end{array}$ & & & \\
\hline & x Generation of odors. & & & \\
\hline & $\begin{array}{l}x \text { High hydraulic residence } \\
\text { times. }\end{array}$ & & & \\
\hline & x Generally, high CAPEX. & & & \\
\hline & $\begin{array}{l}x \text { Generally, post-treatment is } \\
\text { required. }\end{array}$ & & & \\
\hline & $\begin{array}{l}\times \text { Additional costs for the } \\
\text { treatment of the sludge. }\end{array}$ & & & \\
\hline & $\begin{array}{l}x \text { Slight deviations from the } \\
\text { optimal conditions might } \\
\text { result in destabilization. }\end{array}$ & & & \\
\hline
\end{tabular}




\begin{tabular}{|c|c|c|c|c|}
\hline Technology & $\begin{array}{l}\text { Advantages }(\sqrt{ }) \\
\text { Disadvantages }(x) \\
\text { Gaps/Potential to exploit }(\bullet)\end{array}$ & Category & $\begin{array}{l}\text { Common or } \\
\text { Potencial } \\
\text { Industry Sector }\end{array}$ & Source \\
\hline & $\begin{array}{l}\text { - Improve the process } \\
\text { reliability. } \\
\text { - Control of odor production. } \\
\text { - A better understanding of } \\
\text { refractory organic } \\
\text { degradation. } \\
\text { - Reduce hydraulic residence } \\
\text { time. } \\
\text { - Reduce sensibility. } \\
\text { - A better understanding of } \\
\text { fundamentals (microbiology } \\
\text { and biochemical } \\
\text { components). } \\
\text { - Improve the control of the } \\
\text { system. } \\
\text { - Improve the performance in } \\
\text { those cases of treatment of } \\
\text { high solids or high } \\
\text { fat/protein content } \\
\text { wastewater. } \\
\text { - Control of pathogens. } \\
\text { - Improve biogas quality. } \\
\text { - Reuse for the sludge } \\
\text { produced. }\end{array}$ & & & \\
\hline
\end{tabular}




\begin{tabular}{|c|c|c|c|c|}
\hline Technology & $\begin{array}{l}\text { Advantages }(\sqrt{ }) ; \\
\text { Disadvantages }(\mathbf{x}) \text {; } \\
\text { Gaps/Potential to exploit }(\cdot) \text {. }\end{array}$ & Category & $\begin{array}{l}\text { Common or } \\
\text { Potencial } \\
\text { Industry Sector }\end{array}$ & Source \\
\hline $\begin{array}{l}\text { Up-flow anaerobic } \\
\text { sludge blanket } \\
\text { reactor (UASB) }\end{array}$ & $\begin{array}{l}\sqrt{ } \text { High process efficiency, } \\
\text { even with wastewater with a } \\
\text { high organic load. } \\
\sqrt{ } \text { Extensive large-scale } \\
\text { experience. } \\
\sqrt{ } \text { Low energy requirement. } \\
\sqrt{ } \text { Low sludge production. } \\
\sqrt{ } \text { Obtaining fertilizers. } \\
\sqrt{ } \text { Less nutrient requeired } \\
\sqrt{ } \text { Obtaining biogas with } \\
\text { potential use in power } \\
\text { generation. } \\
\sqrt{ } \text { Possibility of plant } \\
\text { shutdown without affecting } \\
\text { the microorganism. } \\
\text { x High Residence times. } \\
\text { x Low effectiveness in } \\
\text { eliminating pathogens and } \\
\text { nutrients. } \\
\text { x High startup times. } \\
\text { x Difficulty in reaching } \\
\text { optimal operating } \\
\text { conditions. } \\
x \text { Generally, high CAPEX. } \\
\text { x Generally, post-treatment is } \\
\text { required. } \\
x \text { Additional costs for the } \\
\text { treatment of the sludge. }\end{array}$ & Established & $\begin{array}{l}\text { Agriculture } \\
\text { Beverage } \\
\text { Distillery } \\
\text { Food } \\
\text { Petroleum } \\
\text { Pulp and Paper } \\
\text { Tannery }\end{array}$ & $\begin{array}{l}\text { (Bhattacharya } \\
\text { et al., 2017; } \\
\text { Hassan et al., } \\
\text { 2013; } \\
\text { Stanbury et } \\
\text { al., 2016; } \\
\text { Varjani et al., } \\
\text { 2020; G. } \\
\text { Yadav et al., } \\
\text { 2021) }\end{array}$ \\
\hline
\end{tabular}




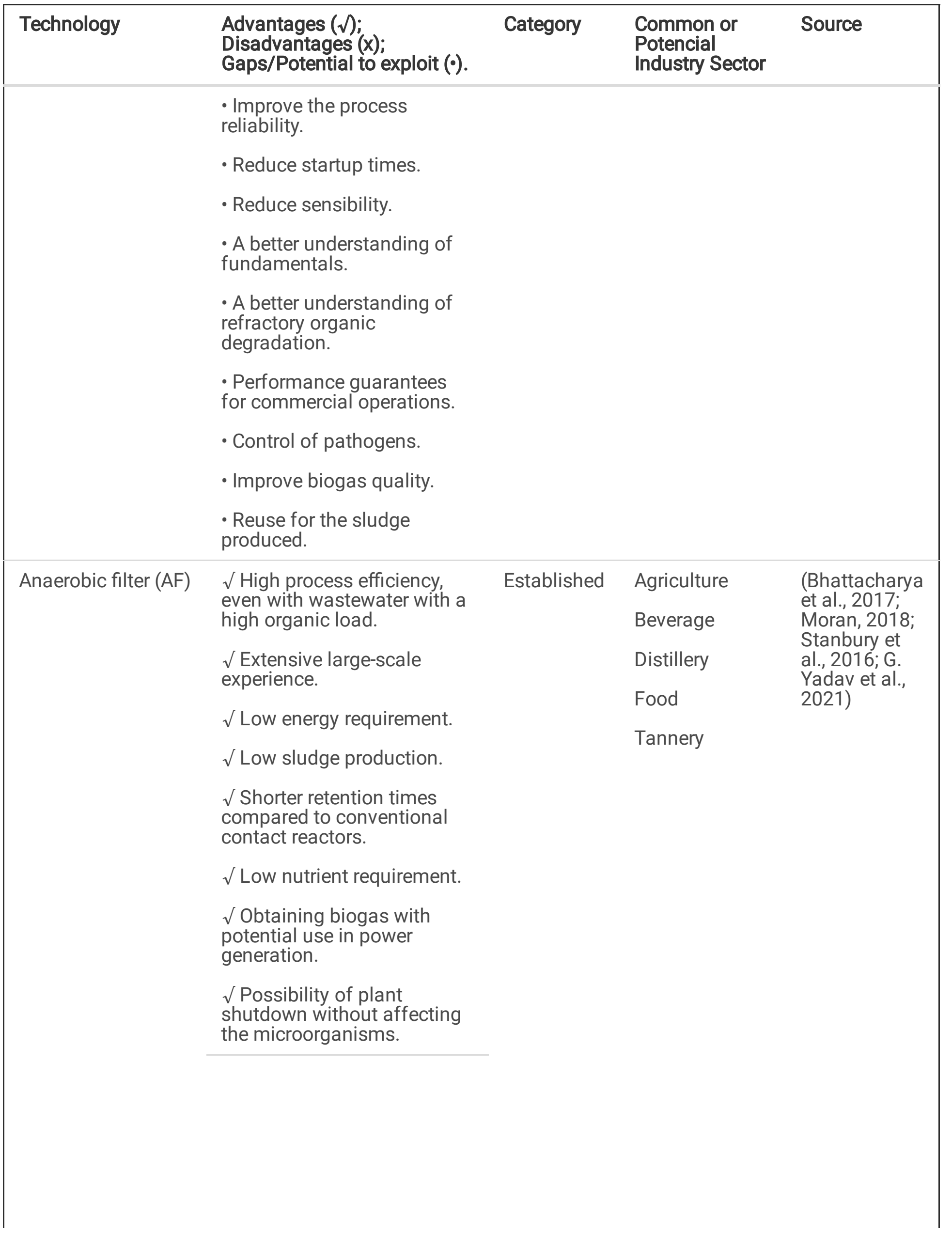




\begin{tabular}{|c|c|c|c|c|}
\hline Technology & $\begin{array}{l}\text { Advantages }(\sqrt{ }) ; \\
\text { Disadvantages }(\mathbf{x}) \text {; } \\
\text { Gaps/Potential to exploit }(\cdot) \text {. }\end{array}$ & Category & $\begin{array}{l}\text { Common or } \\
\text { Potencial } \\
\text { Industry Sector }\end{array}$ & Source \\
\hline & $\begin{array}{l}x \text { Low effectiveness in } \\
\text { eliminating pathogens and } \\
\text { nutrients. }\end{array}$ & & & \\
\hline & x Risk of system clogging. & & & \\
\hline & x Generally, high CAPEX. & & & \\
\hline & $\begin{array}{l}x \text { Generally, post-treatment is } \\
\text { required. }\end{array}$ & & & \\
\hline & $\begin{array}{l}x \text { Additional costs for the } \\
\text { treatment of the sludge. }\end{array}$ & & & \\
\hline & - Process reliability. & & & \\
\hline & $\begin{array}{l}\text { - A better understanding of } \\
\text { fundamentals. }\end{array}$ & & & \\
\hline & $\begin{array}{l}\text { - A better understanding of } \\
\text { refractory organic } \\
\text { degradation. }\end{array}$ & & & \\
\hline & $\begin{array}{l}\text { - Performance guarantees } \\
\text { for commercial operations. }\end{array}$ & & & \\
\hline & • Control of pathogens. & & & \\
\hline & - Improve biogas quality. & & & \\
\hline \multirow{9}{*}{$\begin{array}{l}\text { Anaerobic fluidized } \\
\text { bed reactor (RALF) }\end{array}$} & \multirow{2}{*}{$\begin{array}{l}\sqrt{ } \text { High process efficiency, } \\
\text { even with wastewater with a } \\
\text { high organic load. }\end{array}$} & \multirow[t]{9}{*}{ Established } & Agrochemical & \multirow{9}{*}{$\begin{array}{l}\text { (Bhattacharya } \\
\text { et al., 2017; } \\
\text { Hassan et al., } \\
\text { 2013; G. } \\
\text { Yadav et al., } \\
\text { 2021) }\end{array}$} \\
\hline & & & Chemical & \\
\hline & $\sqrt{ }$ Low energy requirement. & & Distillery & \\
\hline & $\sqrt{ }$ Low sludge production. & & Petroleum & \\
\hline & $\sqrt{ }$ Low nutrient requirement. & & & \\
\hline & $\begin{array}{l}\sqrt{ } \text { Obtaining biogas with } \\
\text { potential use in power } \\
\text { generation. }\end{array}$ & & & \\
\hline & $\begin{array}{l}\sqrt{ } \text { Possibility of plant } \\
\text { shutdown without affecting } \\
\text { the microorganisms. }\end{array}$ & & & \\
\hline & $\begin{array}{l}\sqrt{ } \text { Shorter retention times } \\
\text { compared to conventional } \\
\text { contact reactors. }\end{array}$ & & & \\
\hline & $\sqrt{ }$ Greater COD removal. & & & \\
\hline
\end{tabular}




\begin{tabular}{|c|c|c|c|c|}
\hline Technology & $\begin{array}{l}\text { Advantages }(\sqrt{ }) ; \\
\text { Disadvantages }(\mathrm{x}) \text {; } \\
\text { Gaps/Potential to exploit }(\cdot) \text {. }\end{array}$ & Category & $\begin{array}{l}\text { Common or } \\
\text { Potencial } \\
\text { Industry Sector }\end{array}$ & Source \\
\hline & $\begin{array}{l}x \text { Low effectiveness in } \\
\text { eliminating pathogens and } \\
\text { nutrients. }\end{array}$ & & & \\
\hline & $\begin{array}{l}\text { x Limited large-scale } \\
\text { experience. }\end{array}$ & & & \\
\hline & $\begin{array}{l}\text { x Difficulty in reaching } \\
\text { optimal operating } \\
\text { conditions. }\end{array}$ & & & \\
\hline & x High CAPEX. & & & \\
\hline & $\begin{array}{l}x \text { Generally, post-treatment is } \\
\text { required. }\end{array}$ & & & \\
\hline & $\begin{array}{l}\text { x Additional costs for the } \\
\text { treatment of the sludge. }\end{array}$ & & & \\
\hline & - Process reliability. & & & \\
\hline & - Control of odor production. & & & \\
\hline & $\begin{array}{l}\text { - A better understanding of } \\
\text { fundamentals. }\end{array}$ & & & \\
\hline & $\begin{array}{l}\text { - A better understanding of } \\
\text { refractory organic } \\
\text { degradation. }\end{array}$ & & & \\
\hline & - Control of pathogens. & & & \\
\hline & - Large-scale studies. & & & \\
\hline & - Improve biogas quality. & & & \\
\hline & $\begin{array}{l}\text { - Reuse for the sludge } \\
\text { produced. }\end{array}$ & & & \\
\hline
\end{tabular}




\begin{tabular}{|c|c|c|c|c|}
\hline Technology & $\begin{array}{l}\text { Advantages }(\sqrt{ }) ; \\
\text { Disadvantages }(\mathbf{x}) \text {; } \\
\text { Gaps/Potential to exploit ( } \bullet)\end{array}$ & Category & $\begin{array}{l}\text { Common or } \\
\text { Potencial } \\
\text { Industry Sector }\end{array}$ & Source \\
\hline \multirow{18}{*}{$\begin{array}{l}\text { Nutrient Digester: } \\
\text { Nitrogen removal } \\
\text { bioreactor (NRB) } \\
\text { and Phosphorus } \\
\text { removal bioreactor } \\
\text { (PRB). }\end{array}$} & \multirow{5}{*}{$\begin{array}{l}\sqrt{ } \text { Extensive large-scale } \\
\text { experience. } \\
\sqrt{ } \text { Improvement of the quality } \\
\text { of the treated water. } \\
\sqrt{ } \text { Avoid eutrophication } \\
\text { problems in case of } \\
\text { discharge. }\end{array}$} & \multirow[t]{18}{*}{ Established } & Agriculture & \multirow{18}{*}{$\begin{array}{l}\text { (Ahmed et al., } \\
\text { 2015; } \\
\text { Fernández- } \\
\text { alba, 2006; } \\
\text { Luz \& } \\
\text { Bashan, 2004; } \\
\text { Yorkor \& } \\
\text { Momoh, } \\
\text { 2019) }\end{array}$} \\
\hline & & & Agrochemical & \\
\hline & & & Beverage & \\
\hline & & & Distillery & \\
\hline & & & Food & \\
\hline & $\begin{array}{l}\sqrt{ } \text { The density of the } \\
\text { generated sludge increases. }\end{array}$ & & Tannery & \\
\hline & $\begin{array}{l}\sqrt{ } \text { Decrease in the amount of } \\
\text { sludge generated. }\end{array}$ & & & \\
\hline & $\sqrt{ }$ Greater COD removal. & & & \\
\hline & $\begin{array}{l}\sqrt{ } \text { Potential use in water } \\
\text { recovery and recycling } \\
\text { systems. }\end{array}$ & & & \\
\hline & $\begin{array}{l}\text { x Relatively high energy } \\
\text { requirement. }\end{array}$ & & & \\
\hline & x High CAPEX. & & & \\
\hline & $\begin{array}{l}x \text { Increase in greenhouse gas } \\
\text { emissions, mainly nitrous } \\
\text { oxide. }\end{array}$ & & & \\
\hline & $\begin{array}{l}\text { x Additional costs for the } \\
\text { treatment of the sludge. }\end{array}$ & & & \\
\hline & $\begin{array}{l}\text { - Improve nutrient quality } \\
\text { recovered. }\end{array}$ & & & \\
\hline & $\begin{array}{l}\text { - Reduce sludge volume } \\
\text { produced. }\end{array}$ & & & \\
\hline & $\begin{array}{l}\text { - Reduce greenhouse gas } \\
\text { emissions. }\end{array}$ & & & \\
\hline & - Reduce energy requirement. & & & \\
\hline & $\begin{array}{l}\text { - Reuse for the sludge } \\
\text { produced. }\end{array}$ & & & \\
\hline
\end{tabular}


Table 4

Sludge treatment units: Advantages, disadvantages and gaps/potential to exploit.

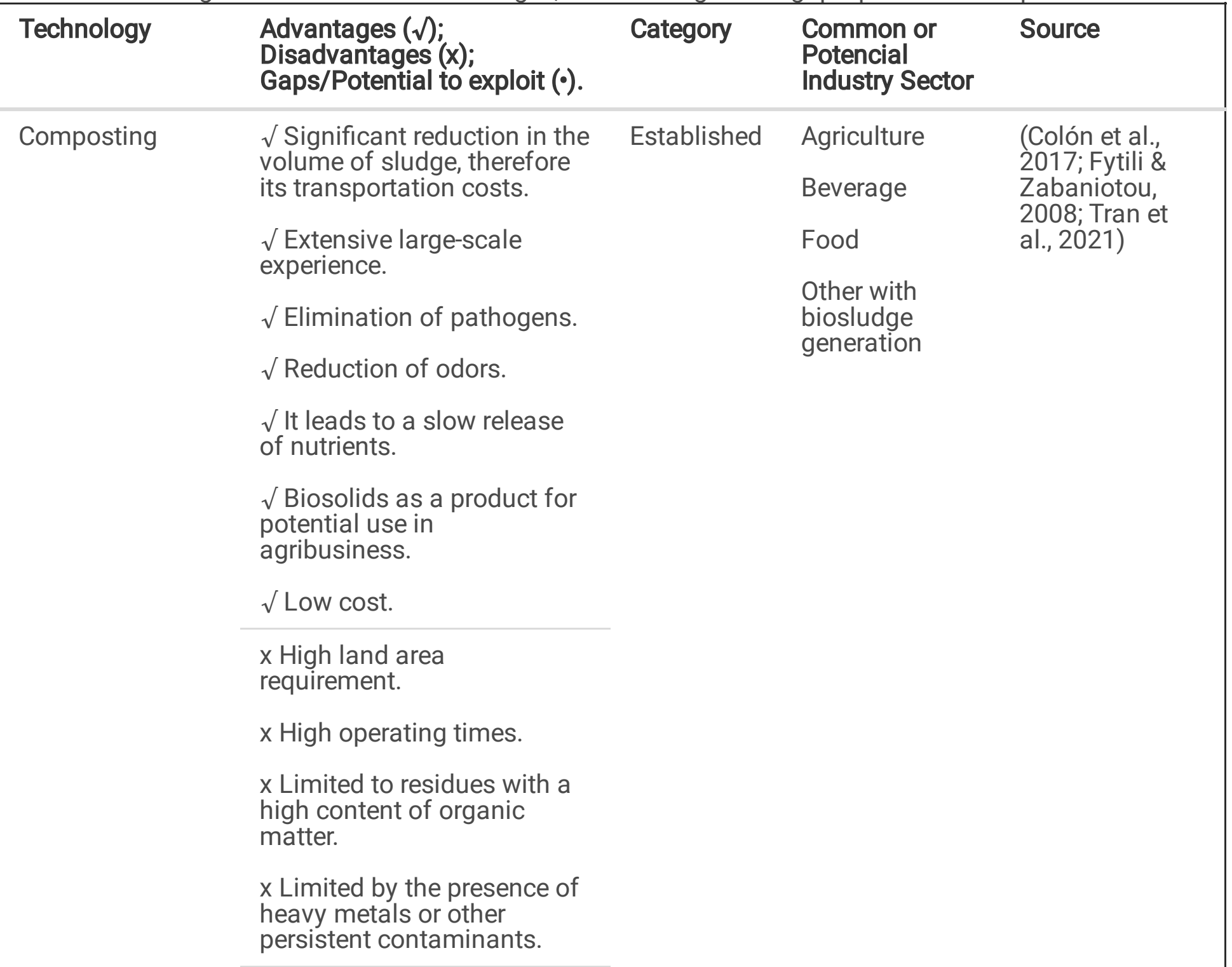




\begin{tabular}{|c|c|c|c|c|}
\hline Technology & $\begin{array}{l}\text { Advantages }(\sqrt{ }) \\
\text { Disadvantages }(x) \text {; } \\
\text { Gaps/Potential to exploit }(\cdot) \text {. }\end{array}$ & Category & $\begin{array}{l}\text { Common or } \\
\text { Potencial } \\
\text { Industry Sector }\end{array}$ & Source \\
\hline & $\begin{array}{l}\text { - Research on optimizing } \\
\text { operational parameters. }\end{array}$ & & & \\
\hline & $\begin{array}{l}\text { - Control strategies (odorous } \\
\text { and toxic gases). }\end{array}$ & & & \\
\hline & $\begin{array}{l}\text { - Understanding the } \\
\text { biodegradation } \\
\text { mechanisms. }\end{array}$ & & & \\
\hline & $\begin{array}{l}\text { - Understanding microbial } \\
\text { diversity and structure } \\
\text { changes along with the } \\
\text { composting processes. }\end{array}$ & & & \\
\hline & $\begin{array}{l}\text { - Reduction in composting } \\
\text { time. }\end{array}$ & & & \\
\hline & $\begin{array}{l}\text { - A better understanding of } \\
\text { fundamentals. }\end{array}$ & & & \\
\hline & - Temperature control. & & & \\
\hline & $\begin{array}{l}\text { - Performance guarantees } \\
\text { for commercial operations } \\
\text { involving solids/sludge from } \\
\text { different IWW and a variety } \\
\text { of pollutants. }\end{array}$ & & & \\
\hline \multirow[t]{6}{*}{ Vermicomposting } & \multirow{2}{*}{$\begin{array}{l}\sqrt{ } \text { Significant reduction in the } \\
\text { volume of sludge, therefore } \\
\text { its transportation costs. }\end{array}$} & \multirow[t]{6}{*}{ Innovative } & Agriculture & \multirow{6}{*}{$\begin{array}{l}\text { (Colón et al., } \\
\text { 2017; Varjani et } \\
\text { al., 2021) }\end{array}$} \\
\hline & & & Beverage & \\
\hline & $\sqrt{ }$ Reduction of odors. & & Food & \\
\hline & $\begin{array}{l}\sqrt{ } \text { Improves water holding } \\
\text { capacity. }\end{array}$ & & \multirow{3}{*}{$\begin{array}{l}\text { Other with } \\
\text { biosludge } \\
\text { generation }\end{array}$} & \\
\hline & $\begin{array}{l}\sqrt{ } \text { It leads to a slow release } \\
\text { of nutrients. }\end{array}$ & & & \\
\hline & $\begin{array}{l}\sqrt{ } \text { Biosolids as a product for } \\
\text { potential use in } \\
\text { agribusiness. }\end{array}$ & & & \\
\hline
\end{tabular}


Advantages $(\sqrt{ })$;

Disadvantages $(x)$;

Gaps/Potential to exploit ( $)$.

$\mathrm{x}$ High land area requirement.

$x$ There is no elimination of pathogens.

x High operating times.

$x$ Not applicable in sludge with low content of organic matter.

$x$ Limited by the presence of heavy metals or other persistent contaminants.

$x$ Limited to residues with a high content of organic matter.

$x$ Suitable growth conditions for worms should be maintained

- Research on optimizing operational parameters.

- Understanding the biodegradation mechanisms.

- Understanding microbial diversity and structure changes along with the vermicomposting processes.

- Reduction of process time.

- A better understanding of fundamentals.

- Temperature control.

- Performance guarantees for commercial operations involving solids/sludge from different IWW and a variety of pollutants. 


\begin{tabular}{|c|c|c|c|c|}
\hline Technology & $\begin{array}{l}\text { Advantages }(\sqrt{ }) ; \\
\text { Disadvantages }(\mathbf{x}) \text {; } \\
\text { Gaps/Potential to exploit (•). }\end{array}$ & Category & $\begin{array}{l}\text { Common or } \\
\text { Potencial } \\
\text { Industry Sector }\end{array}$ & Source \\
\hline $\begin{array}{l}\text { Anaerobic } \\
\text { digester }\end{array}$ & $\begin{array}{l}\sqrt{ } \text { High process efficiency. } \\
\sqrt{ } \text { Extensive large-scale } \\
\text { experience. } \\
\sqrt{ } \text { Significant reduction in the } \\
\text { volume of sludge. } \\
\sqrt{ } \text { Obtaining biogas with } \\
\text { potential use in energy } \\
\text { generation. } \\
\sqrt{ } \text { Obtaining fertilizers. } \\
\sqrt{ } \text { Possibility of plant } \\
\text { shutdown without affecting } \\
\text { the microorganisms. } \\
\sqrt{ } \text { Generally, low OPEX. } \\
x \text { Low effectiveness in } \\
\text { eliminating pathogens and } \\
\text { nutrients. } \\
x \text { Generation of odors. } \\
x \text { High residence times. } \\
x \text { Generally, high CAPEX. } \\
x \text { Generally, post-treatment is } \\
\text { required. } \\
x \text { Additional costs for the } \\
\text { treatment of the sludge. } \\
x \text { Slight in range of } \\
\text { temperature control. } \\
x \text { Limited to residues with a } \\
\text { high content of organic } \\
\text { matter. }\end{array}$ & Established & $\begin{array}{l}\text { Agriculture } \\
\text { Beverage } \\
\text { Food } \\
\text { Other with } \\
\text { biosludge } \\
\text { generation }\end{array}$ & $\begin{array}{l}\text { (Colón et al., } \\
\text { 2017; Varjani et } \\
\text { al., 2021) }\end{array}$ \\
\hline
\end{tabular}




\begin{tabular}{|c|c|c|c|c|}
\hline Technology & $\begin{array}{l}\text { Advantages }(\sqrt{ }) \\
\text { Disadvantages }(x) \text {; } \\
\text { Gaps/Potential to exploit }(\bullet)\end{array}$ & Category & $\begin{array}{l}\text { Common or } \\
\text { Potencial } \\
\text { Industry Sector }\end{array}$ & Source \\
\hline & $\begin{array}{l}\text { - Reduce startup times. } \\
\text { - Reduce sensibility. } \\
\text { - Control of odor production. } \\
\text { - Reduce hydraulic residence } \\
\text { time. } \\
\text { - A better understanding of } \\
\text { fundamentals } \\
\text { - Improve the control of the } \\
\text { system. } \\
\text { - Improve Biogas quality. } \\
\text { - Temperature control. } \\
\text { Performance guarantees for } \\
\text { commercial operations } \\
\text { involving solids/sludge from } \\
\text { different IWW and a variety } \\
\text { of pollutants. }\end{array}$ & & & \\
\hline $\begin{array}{l}\text { Acidic or alkaline } \\
\text { hydrolysis }\end{array}$ & $\begin{array}{l}\sqrt{ } \text { High recovery. } \\
\sqrt{ } \text { Reduction of waste } \\
\text { volume. } \\
\sqrt{ } \text { Destruction of pathogens. } \\
x \text { High consumption of } \\
\text { chemicals. } \\
x \text { High energy requirement. } \\
x \text { Limited capacity. } \\
x \text { Hydrolysis Agent is } \\
\text { required. }\end{array}$ & Established & $\begin{array}{l}\text { Batteries } \\
\text { Chemicals }\end{array}$ & $\begin{array}{l}\text { (Guerra- } \\
\text { Rodríguez et al., } \\
\text { 2020) }\end{array}$ \\
\hline
\end{tabular}




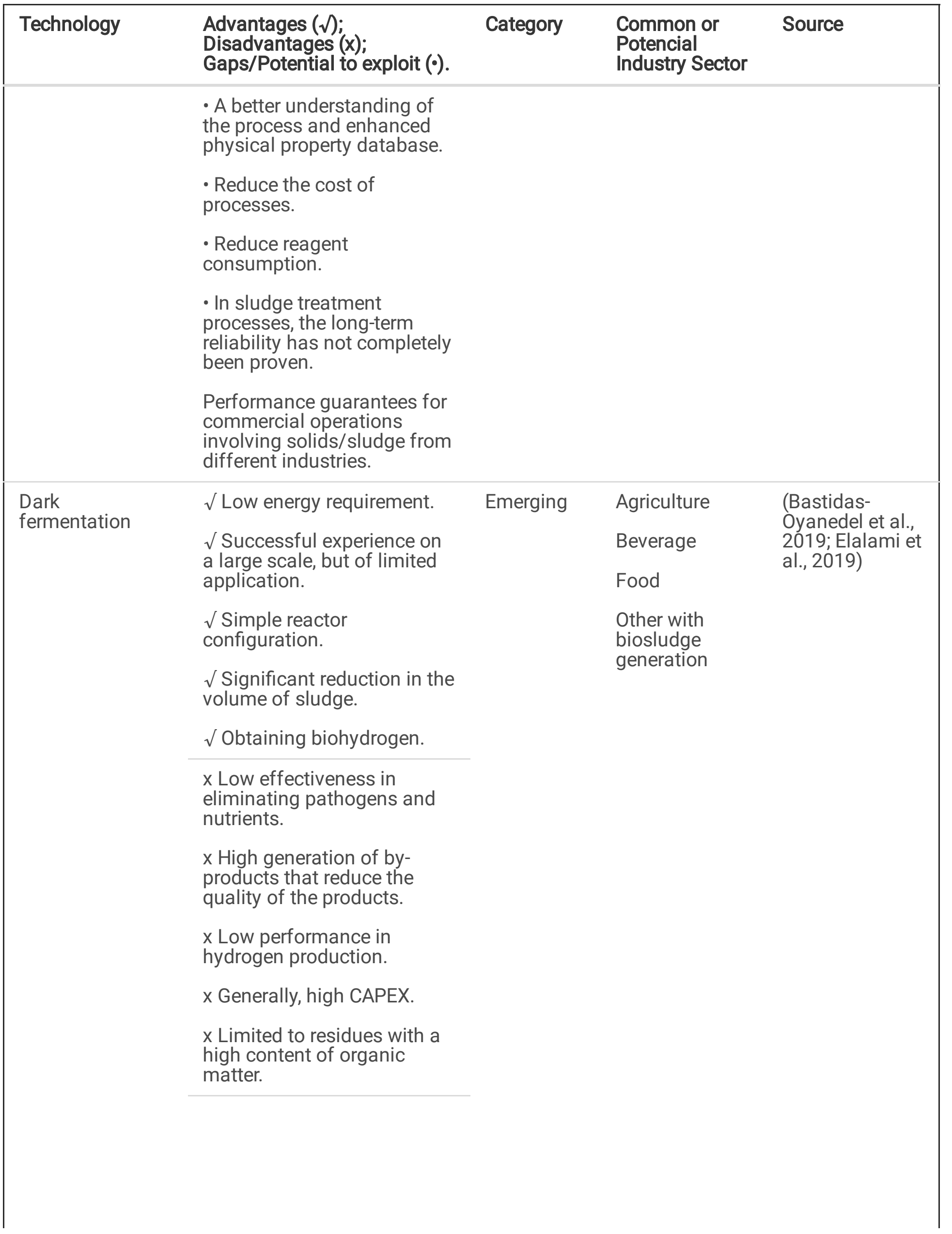




\begin{tabular}{|c|c|c|c|c|}
\hline \multirow[t]{6}{*}{ Technology } & $\begin{array}{l}\text { Advantages }(\sqrt{ }) \\
\text { Disadvantages }(\mathrm{x}) \text {; } \\
\text { Gaps/Potential to exploit (•). }\end{array}$ & Category & $\begin{array}{l}\text { Common or } \\
\text { Potencial } \\
\text { Industry Sector }\end{array}$ & Source \\
\hline & $\begin{array}{l}\text { - Integration of dark } \\
\text { fermentation with other } \\
\text { bioprocesses. }\end{array}$ & & & \\
\hline & - Liquid Fuel Production. & & & \\
\hline & • Fine Chemical Production. & & & \\
\hline & - Syngas Dark Fermentation. & & & \\
\hline & - Large-scale development. & & & \\
\hline \multirow{16}{*}{$\begin{array}{l}\text { Hydrothermal } \\
\text { treatment }\end{array}$} & $\sqrt{ }$ High energy and efficiency. & Established & Mining & \multirow{16}{*}{$\begin{array}{l}\text { (Costa et al., } \\
\text { 2020; Wikberg \& } \\
\text { Jermakka, } \\
\text { 2015; } \\
\text { Yoshimura \& } \\
\text { Byrappa, 2008) }\end{array}$} \\
\hline & $\sqrt{ }$ It can use different raw & & Chemical & \\
\hline & malemals at me same tinte. & & Metal & \\
\hline & $\begin{array}{l}\sqrt{ } \text { Unnecessary deployment } \\
\text { of microorganisms and } \\
\text { enzymes. }\end{array}$ & & & \\
\hline & $\begin{array}{l}\sqrt{ } \text { Potential combination with } \\
\text { other techniques. }\end{array}$ & & & \\
\hline & $\begin{array}{l}\text { x High temperatures and } \\
\text { pressure steam are required. }\end{array}$ & & & \\
\hline & $\mathrm{x}$ Coke and tar formation. & & & \\
\hline & $x$ Obstruction of the reactor. & & & \\
\hline & $\begin{array}{l}x \text { Difficulty in recycling and } \\
\text { regenerating the catalysts. }\end{array}$ & & & \\
\hline & x High CAPEX. & & & \\
\hline & $\begin{array}{l}\text { - Reduce temperatures, } \\
\text { pressure steam, or vacuum } \\
\text { requirement. }\end{array}$ & & & \\
\hline & $\begin{array}{l}\text { - Processing of } \\
\text { nanoparticles. }\end{array}$ & & & \\
\hline & $\begin{array}{l}\text { - Multi-energy-hydrothermal } \\
\text { technology }\end{array}$ & & & \\
\hline & - Supercritical hydrothermal. & & & \\
\hline & $\begin{array}{l}\text { - Reduce reagent } \\
\text { consumption. }\end{array}$ & & & \\
\hline & $\begin{array}{l}\text { - Performance guarantees } \\
\text { for commercial operations } \\
\text { involving solids/sludge from } \\
\text { different industries. }\end{array}$ & & & \\
\hline
\end{tabular}




\begin{tabular}{|c|c|c|c|c|}
\hline Technology & $\begin{array}{l}\text { Advantages }(\sqrt{ }) ; \\
\text { Disadvantages }(\mathbf{x}) \text {; } \\
\text { Gaps/Potential to exploit (·). }\end{array}$ & Category & $\begin{array}{l}\text { Common or } \\
\text { Potencial } \\
\text { Industry Sector }\end{array}$ & Source \\
\hline \multirow{17}{*}{$\begin{array}{l}\text { Bioelectric } \\
\text { systems (BES) }\end{array}$} & \multirow{6}{*}{$\begin{array}{l}\sqrt{ } \text { Generation of } \\
\text { biohydrogen. } \\
\sqrt{ } \text { Power generation. } \\
\sqrt{ } \text { Effective COD removal. } \\
\sqrt{ } \text { Greenhouse gas emissions } \\
\text { control. } \\
\sqrt{ } \text { Polishing wastewaters. }\end{array}$} & \multirow[t]{17}{*}{ Emerging } & \multirow{17}{*}{$\begin{array}{l}\text { Agriculture } \\
\text { Beverage } \\
\text { Food }\end{array}$} & \multirow{17}{*}{$\begin{array}{l}\text { (Cecconet et al., } \\
\text { 2020; Elalami et } \\
\text { al., 2019; Zhang } \\
\text { \& Tremblay, } \\
\text { 2016) }\end{array}$} \\
\hline & & & & \\
\hline & & & & \\
\hline & & & & \\
\hline & & & & \\
\hline & & & & \\
\hline & $\begin{array}{l}\mathrm{x} \text { Energy to stimulate } \\
\text { microbial activity is required. }\end{array}$ & & & \\
\hline & $\begin{array}{l}x \text { Low production rate and } \\
\text { limited efficiency. }\end{array}$ & & & \\
\hline & $\begin{array}{l}x \text { Long residence times are } \\
\text { required. }\end{array}$ & & & \\
\hline & $\begin{array}{l}\text { x Low or no large-scale } \\
\text { experience. }\end{array}$ & & & \\
\hline & -Water desalination. & & & \\
\hline & - IWW treatment. & & & \\
\hline & - Hydrogen production. & & & \\
\hline & - Large-scale development. & & & \\
\hline & $\begin{array}{l}\text { - A better understanding of } \\
\text { electroactive microbial } \\
\text { catalyst, electrochemical } \\
\text { reactor components, and } \\
\text { design. }\end{array}$ & & & \\
\hline & $\begin{array}{l}\text { - Development of models for } \\
\text { proper design and approach. }\end{array}$ & & & \\
\hline & $\begin{array}{l}\text { - Robust and proper BES } \\
\text { design. }\end{array}$ & & & \\
\hline
\end{tabular}




\begin{tabular}{|c|c|c|c|c|}
\hline Technology & $\begin{array}{l}\text { Advantages }(\sqrt{ }) ; \\
\text { Disadvantages }(\mathbf{x}) \text {; } \\
\text { Gaps/Potential to exploit ( } \cdot \text {. }\end{array}$ & Category & $\begin{array}{l}\text { Common or } \\
\text { Potencial } \\
\text { Industry Sector }\end{array}$ & Source \\
\hline Incineration & $\begin{array}{l}\sqrt{ } \text { Extensive large-scale } \\
\text { experience. } \\
\sqrt{ } \text { For biological sludge, its } \\
\text { calorific value is relatively } \\
\text { high, offering the opportunity } \\
\text { for energy recovery. } \\
\sqrt{ } \text { Minimization of odors. } \\
\sqrt{ } \text { Thermal destruction of } \\
\text { toxic organic compounds. } \\
\sqrt{ } \text { Ability to deal with a vast } \\
\text { diversity of wastes. } \\
\text { x It does not constitute a } \\
\text { method of total disposal of } \\
\text { the waste since } 30 \% \text { of this } \\
\text { remains as ash. } \\
\text { x High energy requirement. } \\
\text { x Requirement of a post- } \\
\text { treatment for the resulting } \\
\text { ashes. } \\
\text { x Requirement of a } \\
\text { sophisticated air quality } \\
\text { control system. } \\
x \text { High CAPEX and OPEX. } \\
\text { x Dehydration requirement. }\end{array}$ & Established & $\begin{array}{l}\text { Agrochemical } \\
\text { Batteries } \\
\text { Chemical } \\
\text { Electronics } \\
\text { Medical } \\
\text { Petroleum } \\
\text { Pharmaceutical } \\
\text { Plastics } \\
\text { Tannery }\end{array}$ & $\begin{array}{l}\text { (Colón et al., } \\
\text { 2017; Fytili \& } \\
\text { Zabaniotou, } \\
\text { 2008; Goli et al., } \\
\text { 2021; Ma \& } \\
\text { Rosen, 2021) }\end{array}$ \\
\hline
\end{tabular}




\begin{tabular}{|c|c|c|c|c|}
\hline Technology & $\begin{array}{l}\text { Advantages }(\sqrt{ }) ; \\
\text { Disadvantages }(x) \text {; } \\
\text { Gaps/Potential to exploit }(\cdot)\end{array}$ & Category & $\begin{array}{l}\text { Common or } \\
\text { Potencial } \\
\text { Industry Sector }\end{array}$ & Source \\
\hline & $\begin{array}{l}\text { - Reuse for the slag/ ash } \\
\text { produced during } \\
\text { incineration. } \\
\text { - Heavy metal recovery. } \\
\text { - Nutrient recovery. } \\
\text { - Waste to energy } \\
\text { development. } \\
\text { - Reduce energy requirement. } \\
\text { - Reduce costs. } \\
\text { - Analysis on a case-by-case } \\
\text { basis (based on industry } \\
\text { waste). } \\
\text { - Novel heating approaches } \\
\text { - Control of toxic gas } \\
\text { emissions. }\end{array}$ & & & \\
\hline Pyrolysis & $\begin{array}{l}\sqrt{ } \text { For biological sludge, its } \\
\text { calorific value is relatively } \\
\text { high, offering the opportunity } \\
\text { for energy recovery. } \\
\sqrt{ } \text { Minimization of odors. } \\
\sqrt{ } \text { Thermal destruction of } \\
\text { toxic organic compounds. } \\
\sqrt{ } \text { Low emission. } \\
\sqrt{ } \text { Extensive large-scale } \\
\text { experience, but of limited } \\
\text { application. } \\
\sqrt{ } \text { Usually, the process is self- } \\
\text { sustaining. } \\
\sqrt{ } \text { Production of biofuels and } \\
\text { other materials of interest. } \\
\sqrt{ } \text { Relatively lower operating } \\
\text { temperatures than other } \\
\text { thermal processes. } \\
\sqrt{ } \text { Some elements can be } \\
\text { recovered from the resulting } \\
\text { ash. } \\
\sqrt{ } \text { Minimal amounts of } \\
\text { generated waste. }\end{array}$ & innovative & $\begin{array}{l}\text { Agriculture } \\
\text { Beverage } \\
\text { Food } \\
\text { Other with } \\
\text { biosludge } \\
\text { generation }\end{array}$ & $\begin{array}{l}\text { (Bhatt et al., } \\
\text { 2021; Colón et } \\
\text { al., 2017; } \\
\text { Kamali et al., } \\
\text { 2022; Naqvi et } \\
\text { al., 2021; Ponsa } \\
\text { et al., 2017; } \\
\text { Shyam et al., } \\
\text { 2022; G. Wang } \\
\text { et al., 2020; } \\
\text { Zaharioiu et al., } \\
\text { 2021) }\end{array}$ \\
\hline
\end{tabular}




\begin{tabular}{|c|c|c|c|c|}
\hline Technology & $\begin{array}{l}\text { Advantages }(\sqrt{ }) ; \\
\text { Disadvantages }(\mathrm{x}) \text {; } \\
\text { Gaps/Potential to exploit ( } \bullet \text {. }\end{array}$ & Category & $\begin{array}{l}\text { Common or } \\
\text { Potencial } \\
\text { Industry Sector }\end{array}$ & Source \\
\hline & $\begin{array}{l}x \text { Since a reduced } \\
\text { atmosphere is required, the } \\
\text { products can be highly } \\
\text { corrosive, thus leading to } \\
\text { higher maintenance costs. }\end{array}$ & & & \\
\hline & x High energy requirement. & & & \\
\hline & $x$ High CAPEX and OPEX. & & & \\
\hline & $\begin{array}{l}x \text { Limited to residues with a } \\
\text { high content of organic } \\
\text { matter. }\end{array}$ & & & \\
\hline & $\mathrm{x}$ Dehydration requirement. & & & \\
\hline & - Large-scale development. & & & \\
\hline & $\begin{array}{l}\text { - Improved understanding of } \\
\text { co-pyrolysis mechanism. }\end{array}$ & & & \\
\hline & - Reduce energy requirement. & & & \\
\hline & $\begin{array}{l}\text { - Integration with renewable } \\
\text { energy sources. }\end{array}$ & & & \\
\hline & $\begin{array}{l}\text { - Biochar and other by- } \\
\text { product applications. }\end{array}$ & & & \\
\hline & - Reduce costs. & & & \\
\hline & $\begin{array}{l}\text { - Waste to energy } \\
\text { development. }\end{array}$ & & & \\
\hline & $\begin{array}{l}\text { - Development of efficient } \\
\text { adsorbents. }\end{array}$ & & & \\
\hline & $\begin{array}{l}\text { - Control of Nitrogen } \\
\text { emissions and air pollution. }\end{array}$ & & & \\
\hline & - Novel heating approaches & & & \\
\hline & $\begin{array}{l}\text { - More kinetic evaluation } \\
\text { studies. }\end{array}$ & & & \\
\hline
\end{tabular}




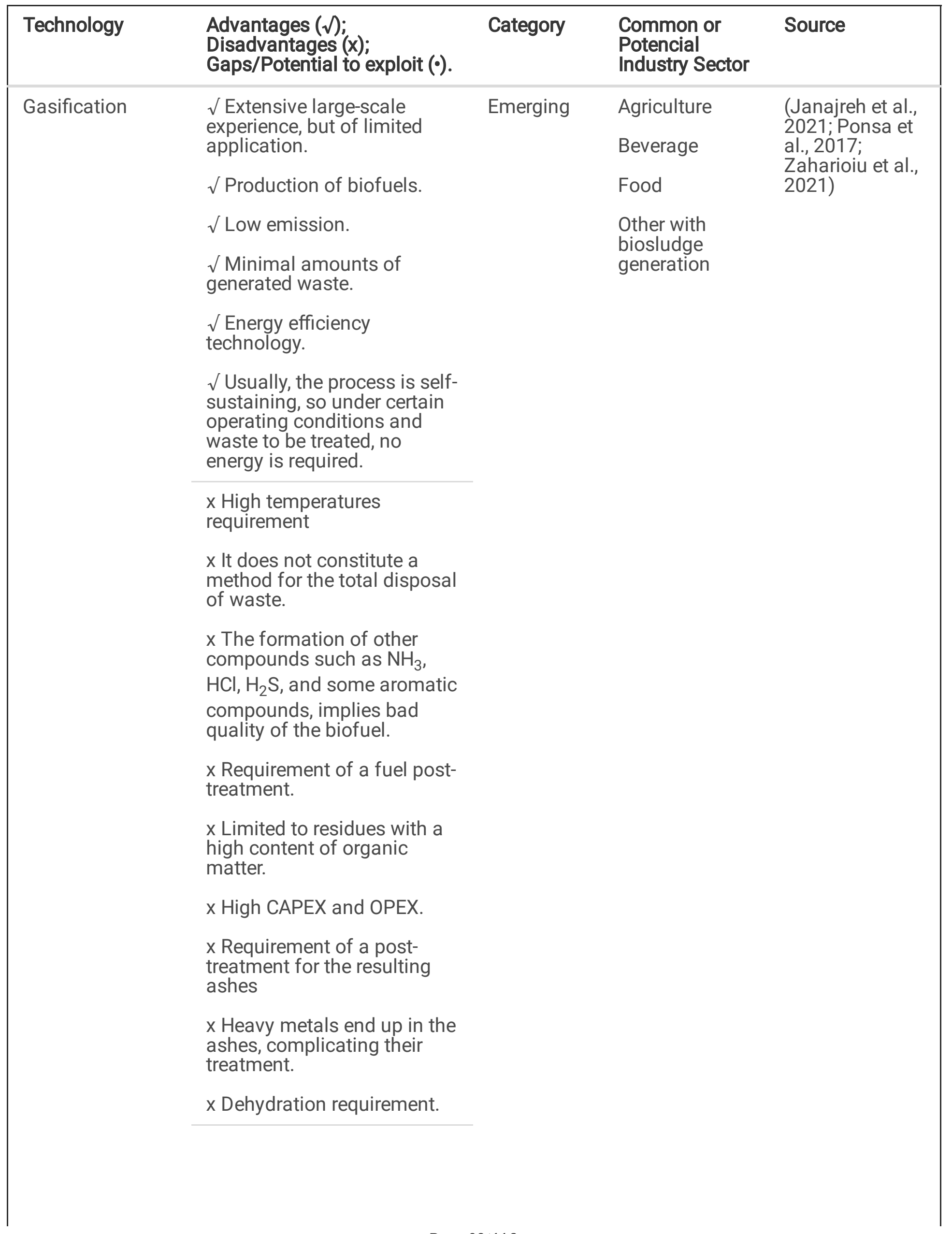




\begin{tabular}{|lllll|}
\hline Technology & $\begin{array}{l}\text { Advantages }(\sqrt{ }) ; \\
\text { Disadvantages }(x) ; \\
\text { Gaps/Potential to exploit }(\bullet) .\end{array}$ & Category & $\begin{array}{l}\text { Common or } \\
\text { Potencial } \\
\text { Industry Sector }\end{array}$ & Source \\
& & & \\
\hline
\end{tabular}

- Waste to energy development.

- Control of air pollution.

- Novel heating approaches.

- Hybrid processes and process intensification that result in enhanced efficiency and efficacy.

- Development of plasma gasification, supercritical water gasification, cogasification.

- Reduce energy requirement.

- Reduce costs.

- Improved understanding of gasification reactions.

- Tar valorization.

- Feedstock versus gasifier type studies.

- Gasifier sizing, and the different zonal influence (drying, combustion, and reduction) studies.

Wet oxidation (WO) $\sqrt{ }$ Extensive large-scale experience, but of limited application.

$\sqrt{ }$ An alternative solution to combustion.

$\sqrt{ }$ The gaseous product does not contain dangerous compounds.

$\sqrt{ }$ The resulting wastewater is constituted of biodegradable compounds and ammonia.

$\begin{array}{lll}\text { Emerging } & \text { Agriculture } & \text { (Fytili \& } \\ & & \text { Zabaniotou, } \\ & \text { Beverage } & \text { 2008; Genc et } \\ & \text { al., 2002; S. } \\ & \text { Pérez-Elvira et } \\ & \text { al., 2006; Sara } \\ & \text { Pérez-Elvira et } \\ & \text { al., 2017) }\end{array}$

(Fytili \& 2008: Genc et al., 2002; S al., 2006; Sara al., 2017) 


\begin{tabular}{|c|c|c|c|c|}
\hline Technology & $\begin{array}{l}\text { Advantages }(\sqrt{ }) \\
\text { Disadvantages }(x) \text {; } \\
\text { Gaps/Potential to exploit }(\cdot) \text {. }\end{array}$ & Category & $\begin{array}{l}\text { Common or } \\
\text { Potencial } \\
\text { Industry Sector }\end{array}$ & Source \\
\hline & $\begin{array}{l}\text { x High energy requirement. } \\
\text { x High OPEX. }\end{array}$ & & & \\
\hline & $\begin{array}{l}x \text { Post-treatment is required } \\
\text { for the final product. }\end{array}$ & & & \\
\hline & - Hybrid processes. & & & \\
\hline & - Reduce costs. & & & \\
\hline & - Reduce energy requirement. & & & \\
\hline & $\begin{array}{l}\text { - Database of successful WO } \\
\text { cases at lab, pilot, and plant- } \\
\text { level should be prepared. }\end{array}$ & & & \\
\hline & $\begin{array}{l}\text { - Database of successful WO } \\
\text { cases at lab, pilot, and plant- } \\
\text { level should be prepared. }\end{array}$ & & & \\
\hline & $\begin{array}{l}\text { - Hybrid processes and } \\
\text { process intensification that } \\
\text { result in enhanced efficiency } \\
\text { and efficacy. }\end{array}$ & & & \\
\hline & $\begin{array}{l}\text { - Performance guarantees } \\
\text { for commercial operations } \\
\text { involving different IWW } \\
\text { sludges and a variety of } \\
\text { pollutants. }\end{array}$ & & & \\
\hline \multirow{7}{*}{$\begin{array}{l}\text { Solidification } \\
\text { Stabilization } \\
\text { (S/S) }\end{array}$} & \multirow{3}{*}{$\begin{array}{l}\sqrt{ } \text { Reduction of pathogens. } \\
\sqrt{ } \text { Reduction of the } \\
\text { availability of metals. }\end{array}$} & \multirow[t]{7}{*}{ Established } & Agriculture & \multirow{7}{*}{$\begin{array}{l}\text { (Colón et al., } \\
\text { 2017; Ponsa et } \\
\text { al., 2017; Wong } \\
\text { \& Selvam, } \\
\text { 2006) }\end{array}$} \\
\hline & & & Beverage & \\
\hline & & & Food & \\
\hline & $\begin{array}{l}\sqrt{ } \text { Extensive large-scale } \\
\text { experience. }\end{array}$ & & Mining & \\
\hline & $\sqrt{ }$ Simple operation. & & & \\
\hline & $\sqrt{ }$ Recovery of nutrients. & & & \\
\hline & $\begin{array}{l}\sqrt{ } \text { Relatively low CAPEX and } \\
\text { OPEX. }\end{array}$ & & & \\
\hline
\end{tabular}




\begin{tabular}{|lllll|}
\hline Technology & $\begin{array}{l}\text { Advantages }(\sqrt{ }) ; \\
\text { Disadvantages }(\mathrm{x}) ; \\
\text { Gaps/Potential to exploit }(\cdot) .\end{array}$ & Category & $\begin{array}{l}\text { Common or } \\
\text { Potencial } \\
\text { Industry Sector }\end{array}$ & Source \\
& & & \\
\hline
\end{tabular}

$x$ High requirement of stabilizing agents.

$x$ Not applicable in sludge with low content of organic matter.

x Drying requirement.

$x$ Limited by the presence of heavy metals or other persistent contaminants.

- Recovery of heavy metals and other pollutants.

- Reduce the reagents requirement.

- Improve nutrient recovery.

- Performance guarantees for commercial operations involving different IWW sludges and a variety of pollutants.

Conventional leaching $\sqrt{ }$ Extensive large-scale experience.

$\sqrt{ }$ Relatively low CAPEX and OPEX.

$\sqrt{ }$ Low energy requirement.

$\sqrt{ }$ Simple and easy-tooperate equipment.

$\sqrt{ }$ Lower water requirement compared to other technologies such as flotation.

x High environmental impact.

x High solvent requirement.

$x$ Requirement of a posttreatment for the solvent.

\section{Established Agrochemical}

Batteries

Chemical

Electronics

Metals

Mining
(Ghorbani et al., 2016; Veit \& Bernardes, 2015) 


\begin{tabular}{|c|c|c|c|c|}
\hline Technology & $\begin{array}{l}\text { Advantages }(\sqrt{ }) \\
\text { Disadvantages }(x) \\
\text { Gaps/Potential to exploit }(\cdot)\end{array}$ & Category & $\begin{array}{l}\text { Common or } \\
\text { Potencial } \\
\text { Industry Sector }\end{array}$ & Source \\
\hline & $\begin{array}{l}\text { - New biodegradable } \\
\text { solvents, suitable for } \\
\text { selective recovery of } \\
\text { pollutants. } \\
\text { - New equipment and } \\
\text { process design for extraction } \\
\text { and recovery. } \\
\text { - Reduce costs. } \\
\text { - Efficient ways to regenerate } \\
\text { and reuse absorbents. } \\
\text { - Performance guarantees } \\
\text { for commercial operations } \\
\text { involving different IWW } \\
\text { sludge and solid wastes. } \\
\text { - A better understanding of } \\
\text { the process and enhanced } \\
\text { physical property database. }\end{array}$ & & & \\
\hline Bioleaching & $\begin{array}{l}\sqrt{ } \text { Lower environmental } \\
\text { impact compared to } \\
\text { conventional absorption } \\
\text { technologies. } \\
\sqrt{ } \text { There is a large-scale } \\
\text { experience for the recovery } \\
\text { of metals but of limited } \\
\text { application. } \\
\text { x Despite having large-scale } \\
\text { experience, it is not a very } \\
\text { widespread technology. } \\
\text { x Removal rate lower than } \\
\text { traditional methods. } \\
\text { x High CAPEX and OPEX. } \\
\text { x Slow process compared to } \\
\text { traditional methods. }\end{array}$ & Emerging & $\begin{array}{l}\text { Batteries } \\
\text { Electronics } \\
\text { Metals } \\
\text { Mining }\end{array}$ & $\begin{array}{l}\text { (Colón et al., } \\
\text { 2017; Johnson, } \\
\text { 2018; Mohanty } \\
\text { et al., 2018; Veit } \\
\text { \& Bernardes, } \\
\text { 2015) }\end{array}$ \\
\hline
\end{tabular}




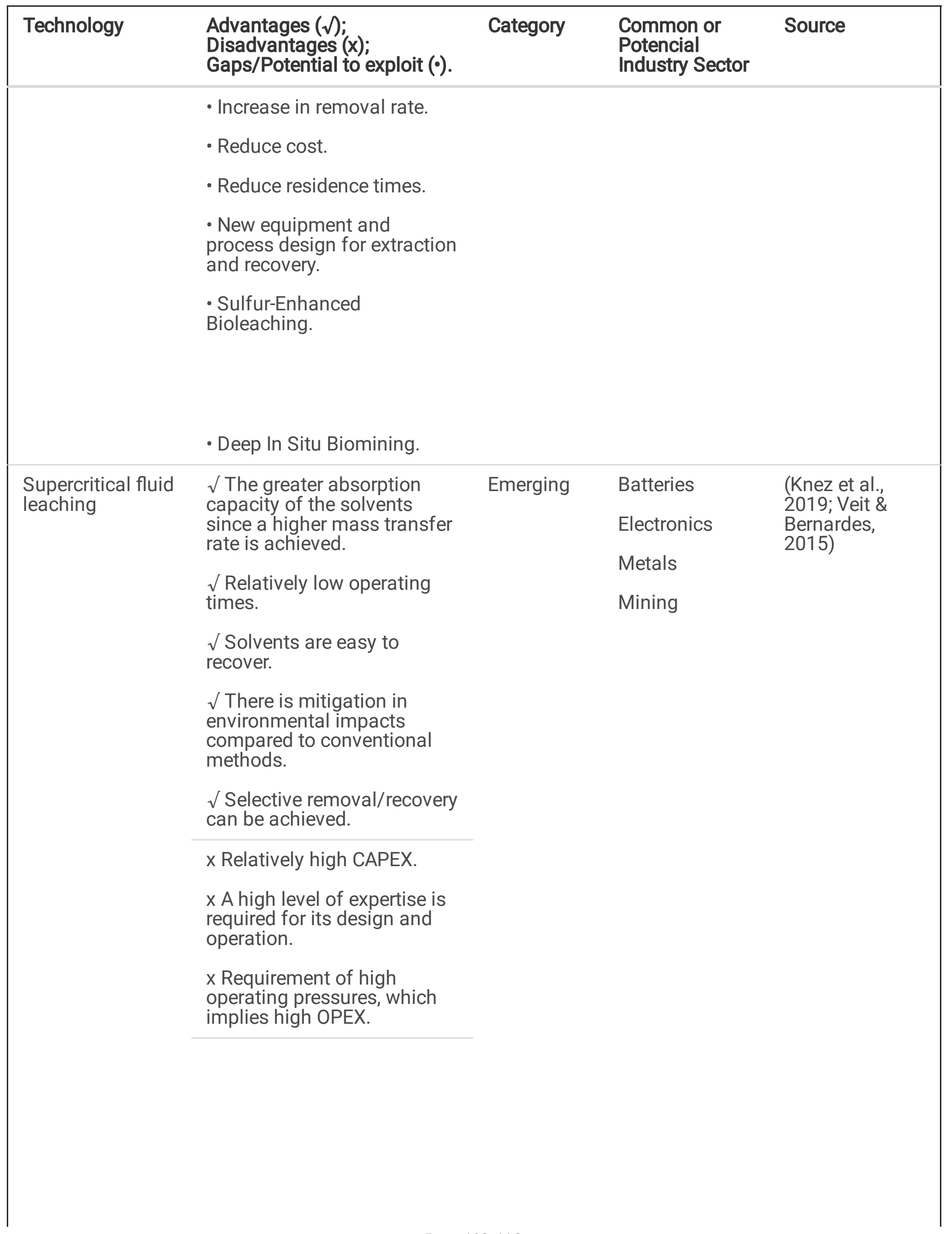




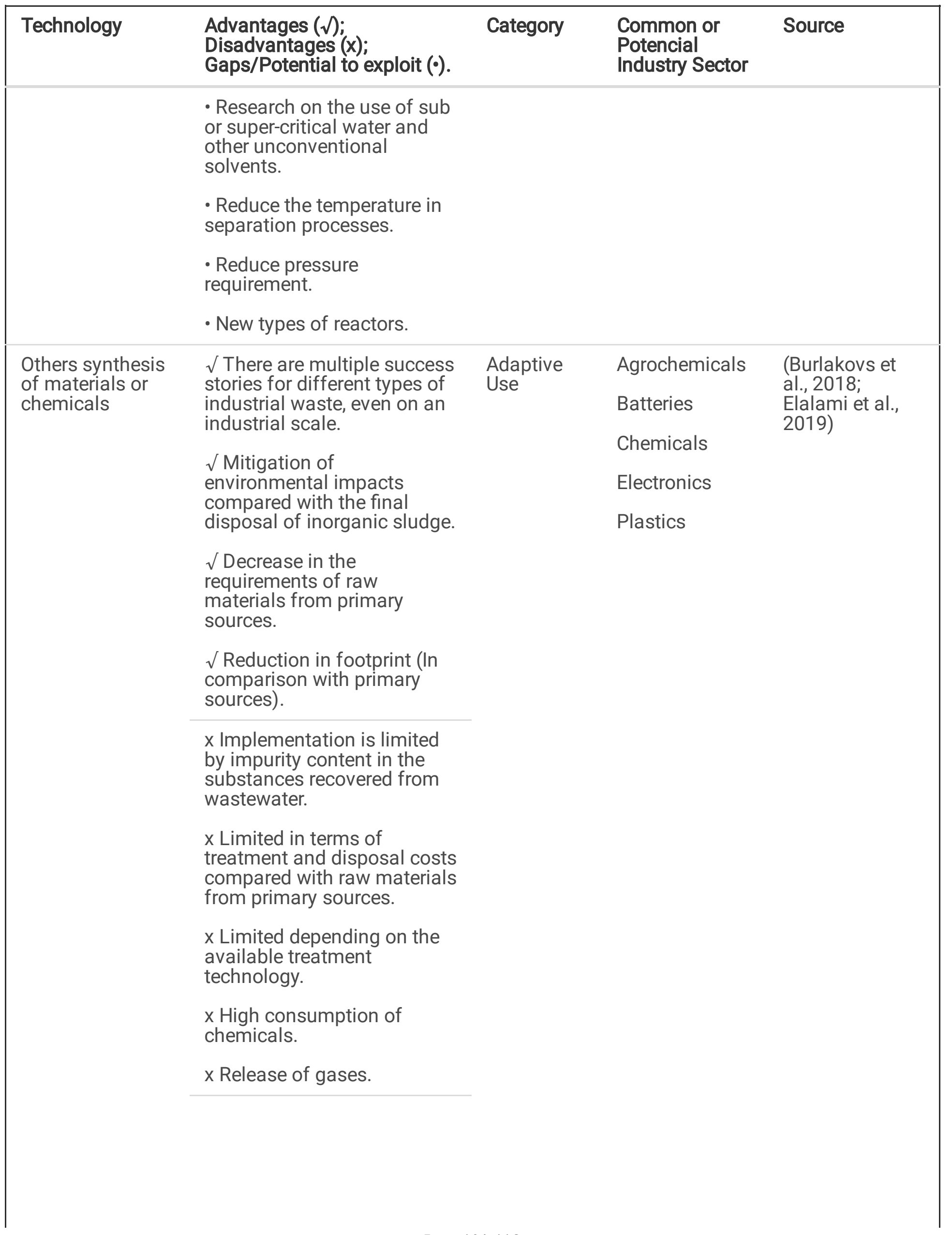




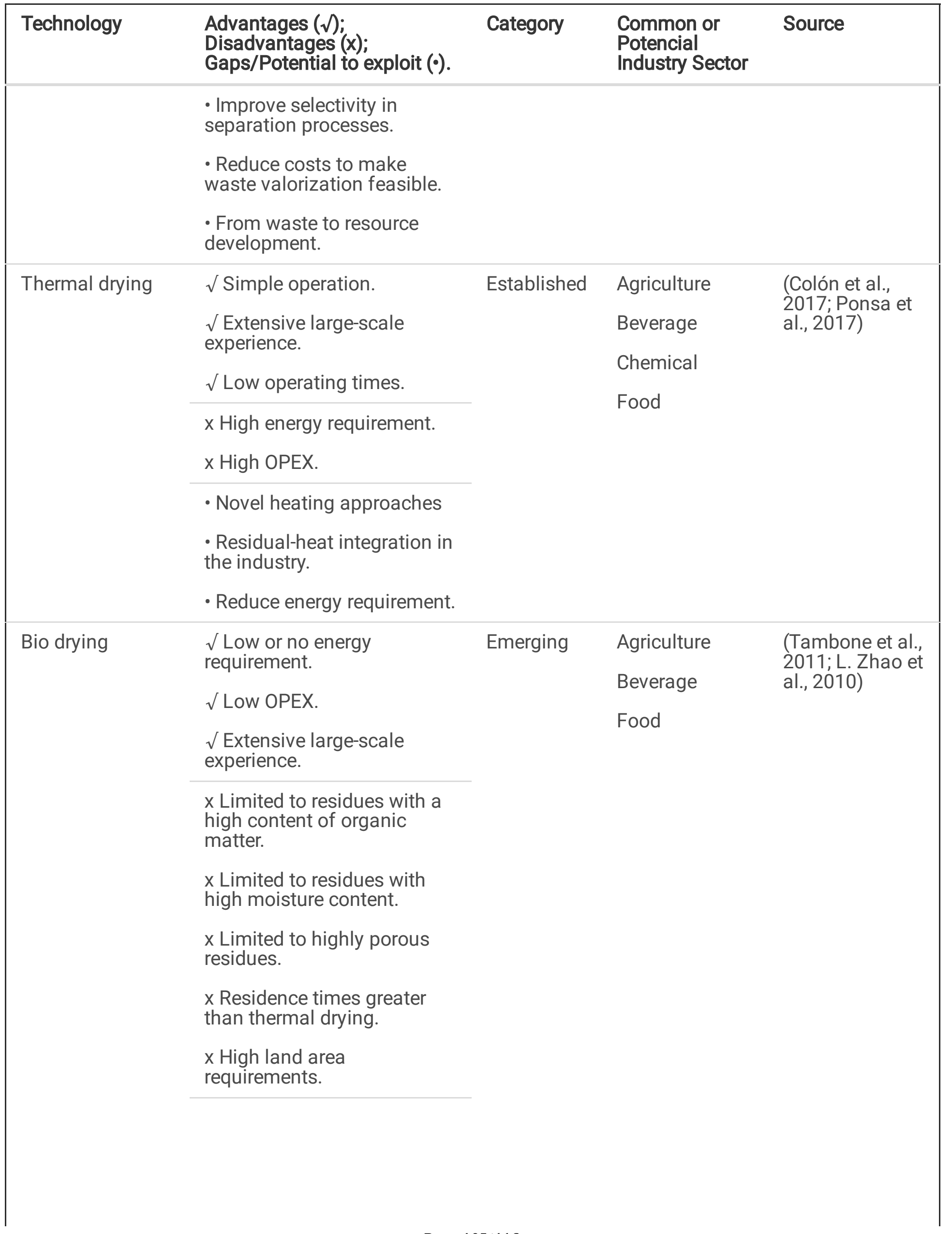




\begin{tabular}{|c|c|c|c|c|}
\hline \multirow[t]{4}{*}{ Technology } & $\begin{array}{l}\text { Advantages }(\sqrt{ }) ; \\
\text { Disadvantages }(\mathrm{x}) \text {; } \\
\text { Gaps/Potential to exploit (•). }\end{array}$ & \multirow[t]{4}{*}{ Category } & \multirow[t]{4}{*}{$\begin{array}{l}\text { Common or } \\
\text { Potencial } \\
\text { Industry Sector }\end{array}$} & \multirow[t]{4}{*}{ Source } \\
\hline & - Reduce residence times. & & & \\
\hline & $\begin{array}{l}\text { - Reduce land area } \\
\text { requirement. }\end{array}$ & & & \\
\hline & $\begin{array}{l}\text { - Study feasibility for } \\
\text { different IWW sludges. }\end{array}$ & & & \\
\hline \multirow[t]{10}{*}{ Solar drying } & $\sqrt{ }$ Low or No power & \multirow[t]{10}{*}{ Emerging } & \multirow{10}{*}{$\begin{array}{l}\text { Agriculture } \\
\text { Beverage } \\
\text { Food }\end{array}$} & \multirow{10}{*}{$\begin{array}{l}\text { (Bennamoun, } \\
2012 ; \\
\text { Kamarulzaman } \\
\text { et al., 2021; } \\
\text { Mohsin et al., } \\
\text { 2011) }\end{array}$} \\
\hline & 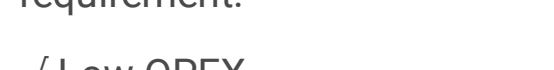 & & & \\
\hline & $\sqrt{ }$ Low OPEX. & & & \\
\hline & $\begin{array}{l}\sqrt{ } \text { Extensive large-scale } \\
\text { experience. }\end{array}$ & & & \\
\hline & $\begin{array}{l}x \text { Residence times longer } \\
\text { than thermal drying. }\end{array}$ & & & \\
\hline & $\begin{array}{l}\text { x High land area } \\
\text { requirements. }\end{array}$ & & & \\
\hline & x Operational limitations. & & & \\
\hline & - Reduce residence times. & & & \\
\hline & $\begin{array}{l}\text { - Reduce land area } \\
\text { requirement. }\end{array}$ & & & \\
\hline & $\begin{array}{l}\text { - Increase efficiency on } \\
\text { designs, especially with the } \\
\text { enhancement of the solar } \\
\text { absorber designs, } \\
\text { configurations and material, } \\
\text { integration of thermal } \\
\text { storage, and applications of } \\
\text { different thermos fluids. }\end{array}$ & & & \\
\hline \multirow{6}{*}{$\begin{array}{l}\text { Cement and } \\
\text { construction } \\
\text { aggregates } \\
\text { production base }\end{array}$} & $\sqrt{ }$ It may imply a significant & \multirow[t]{6}{*}{ Innovative } & \multirow{6}{*}{$\begin{array}{l}\text { Construction } \\
\text { Metal } \\
\text { Mining }\end{array}$} & \multirow{6}{*}{$\begin{array}{l}\text { (Bernal et al., } \\
\text { 2016; Martínez- } \\
\text { Martínez et al., } \\
\text { 2020; } \\
\text { Papatzani, } \\
\text { 2019) }\end{array}$} \\
\hline & & & & \\
\hline & $\begin{array}{l}\sqrt{ } \text { It reduces the requirement } \\
\text { of raw materials from } \\
\text { primary sources. }\end{array}$ & & & \\
\hline & $\begin{array}{l}\sqrt{ } \text { Reduction in the Carbon } \\
\text { footprint (In comparison with } \\
\text { other alternatives). }\end{array}$ & & & \\
\hline & $\begin{array}{l}\sqrt{ } \text { It is considered a green } \\
\text { construction material. }\end{array}$ & & & \\
\hline & $\begin{array}{l}\sqrt{ } \text { It reduces the need for new } \\
\text { landfills. }\end{array}$ & & & \\
\hline
\end{tabular}


$x$ In general, only residues with a high content of lime, silica, alumina, and iron oxide come to be considered viable.

$x$ It could downgrade the quality in terms of mechanical resistance and water adsorption.

$x$ The content of some species may limit the application of waste as a base material in the production of cement or as a construction aggregate.

$x$ The content of metals and other pollutants can be released from the element, implying an environmental impact on the environment.

$x$ It may require pretreatment systems for the removal of contaminants in the waste.

$\mathrm{x}$ It is not considered as such a system of use for valuable components since the technology consists of stabilizing and encapsulating the waste.

- Recycling industry.

- Specifications and guidelines for industry sludge/wastes.

- Improve the quality of industry sludge/wastes to minimize o even improve the final quality in terms of mechanical resistance and water adsorption.

- Improved the understanding of the performance of waste to aggregates or cement production. 


\begin{tabular}{|c|c|c|c|c|}
\hline Technology & $\begin{array}{l}\text { Advantages }(\sqrt{ }) \\
\text { Disadvantages }(\mathbf{x}) \text {; } \\
\text { Gaps/Potential to exploit (•). }\end{array}$ & Category & $\begin{array}{l}\text { Common or } \\
\text { Potencial } \\
\text { Industry Sector }\end{array}$ & Source \\
\hline \multirow{20}{*}{$\begin{array}{l}\text { Disposal in } \\
\text { landfills }\end{array}$} & \multirow{6}{*}{$\begin{array}{l}\sqrt{ } \text { Extensive large-scale } \\
\text { experience. } \\
\sqrt{ } \text { Low OPEX. } \\
\sqrt{ } \text { Easy to operate. } \\
\sqrt{ } \text { Easy to deal with } \\
\text { sludge/waste. }\end{array}$} & \multirow[t]{20}{*}{ Established } & Agrochemical & \multirow{20}{*}{$\begin{array}{l}\text { (Blair \& } \\
\text { Mataraarachchi, } \\
\text { 2021; Varjani et } \\
\text { al., 2021; } \\
\text { Woodard \& } \\
\text { Curran, 2006c) }\end{array}$} \\
\hline & & & Beverages & \\
\hline & & & Chemical & \\
\hline & & & Fond & \\
\hline & & & Medical & \\
\hline & & & & \\
\hline & \multirow{2}{*}{$\begin{array}{l}\mathrm{x} \text { It goes against the } \\
\text { principles of sustainable } \\
\text { development. }\end{array}$} & & Pharmaceutical & \\
\hline & & & Pulp/paper & \\
\hline & x Loss of resources. & & Tannery & \\
\hline & $\begin{array}{l}\mathrm{x} \text { The residue may still } \\
\text { require pre-treatment. }\end{array}$ & & Textile & \\
\hline & $\begin{array}{l}\text { x Requirement of specialized } \\
\text { landfills. }\end{array}$ & & & \\
\hline & x Drying requirement. & & & \\
\hline & $\begin{array}{l}x \text { Leachates are difficult and } \\
\text { expensive to treat. }\end{array}$ & & & \\
\hline & x Pollutes the soil. & & & \\
\hline & $\begin{array}{l}x \text { It could contribute to } \\
\text { groundwater pollution and } \\
\text { deforestation. }\end{array}$ & & & \\
\hline & $\begin{array}{l}\text { - GHG estimation and } \\
\text { reduction. }\end{array}$ & & & \\
\hline & - Leachate treatment. & & & \\
\hline & $\begin{array}{l}\text { - Material recovery after } \\
\text { closure. }\end{array}$ & & & \\
\hline & - Control of infiltration. & & & \\
\hline & - Restoration of soil. & & & \\
\hline
\end{tabular}

\section{Figures}




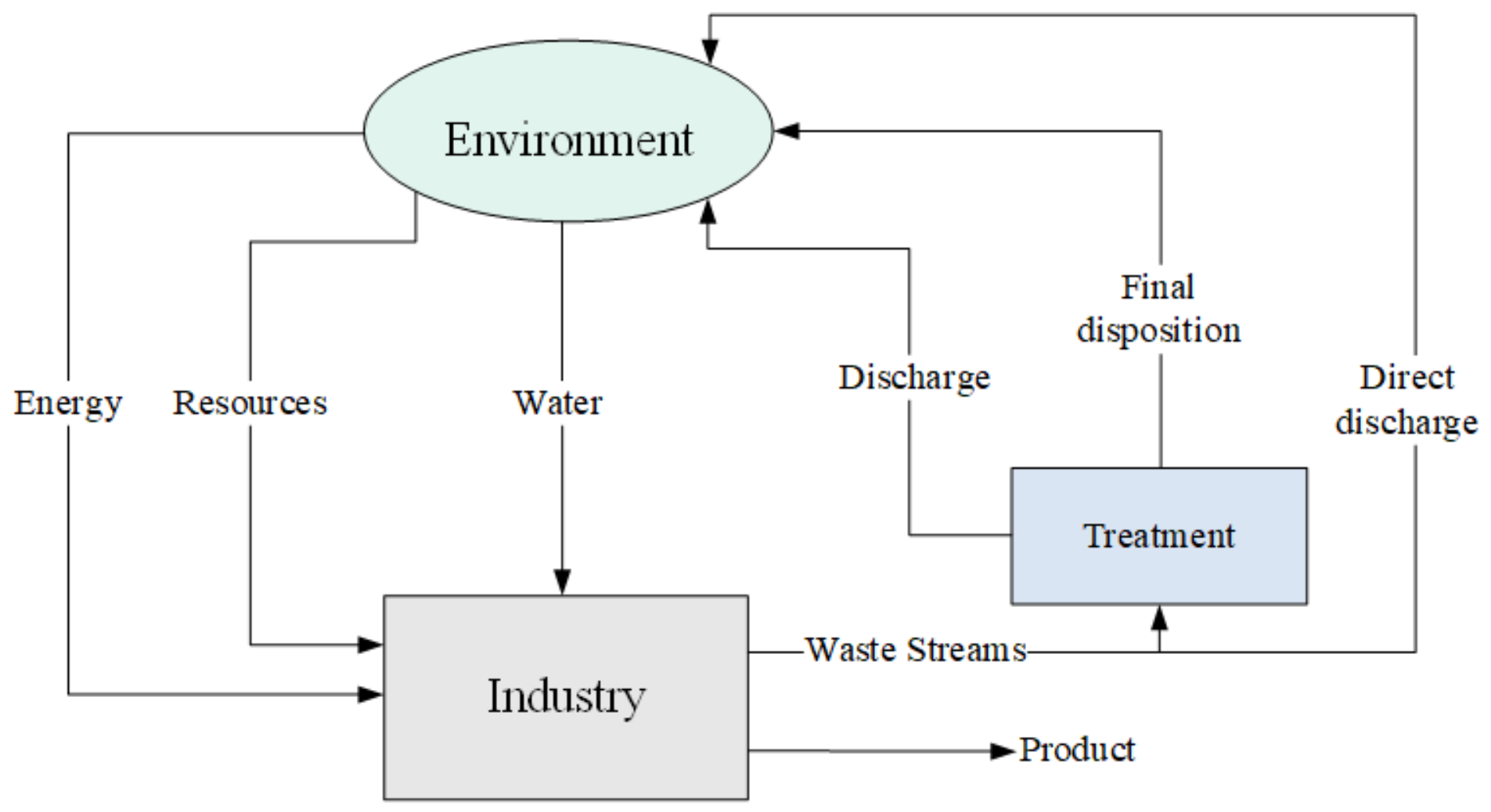

Figure 1

Traditional cycle in industrial process.

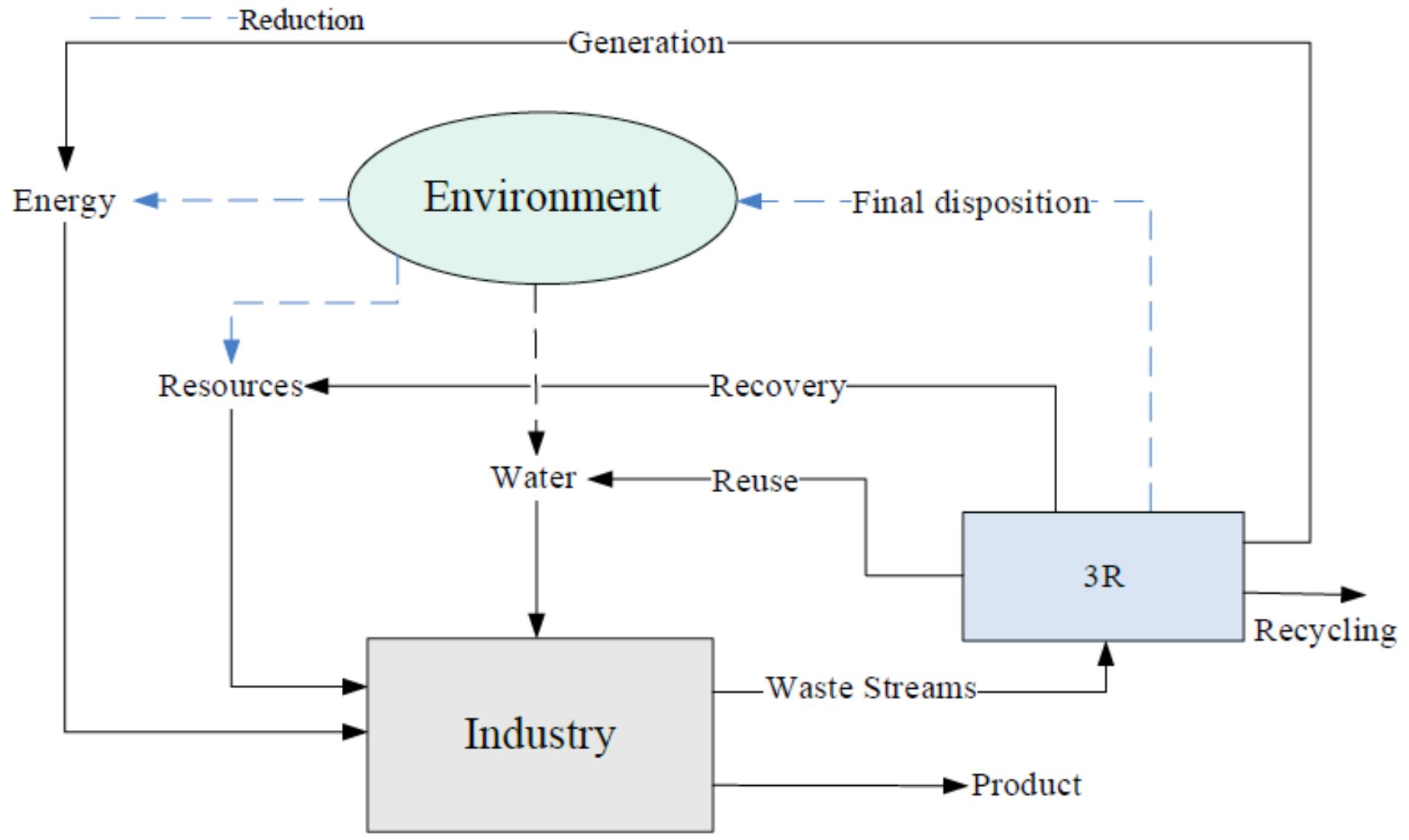


Figure 2

Ideal cycle in industrial process with a 3R scheme WWTP.

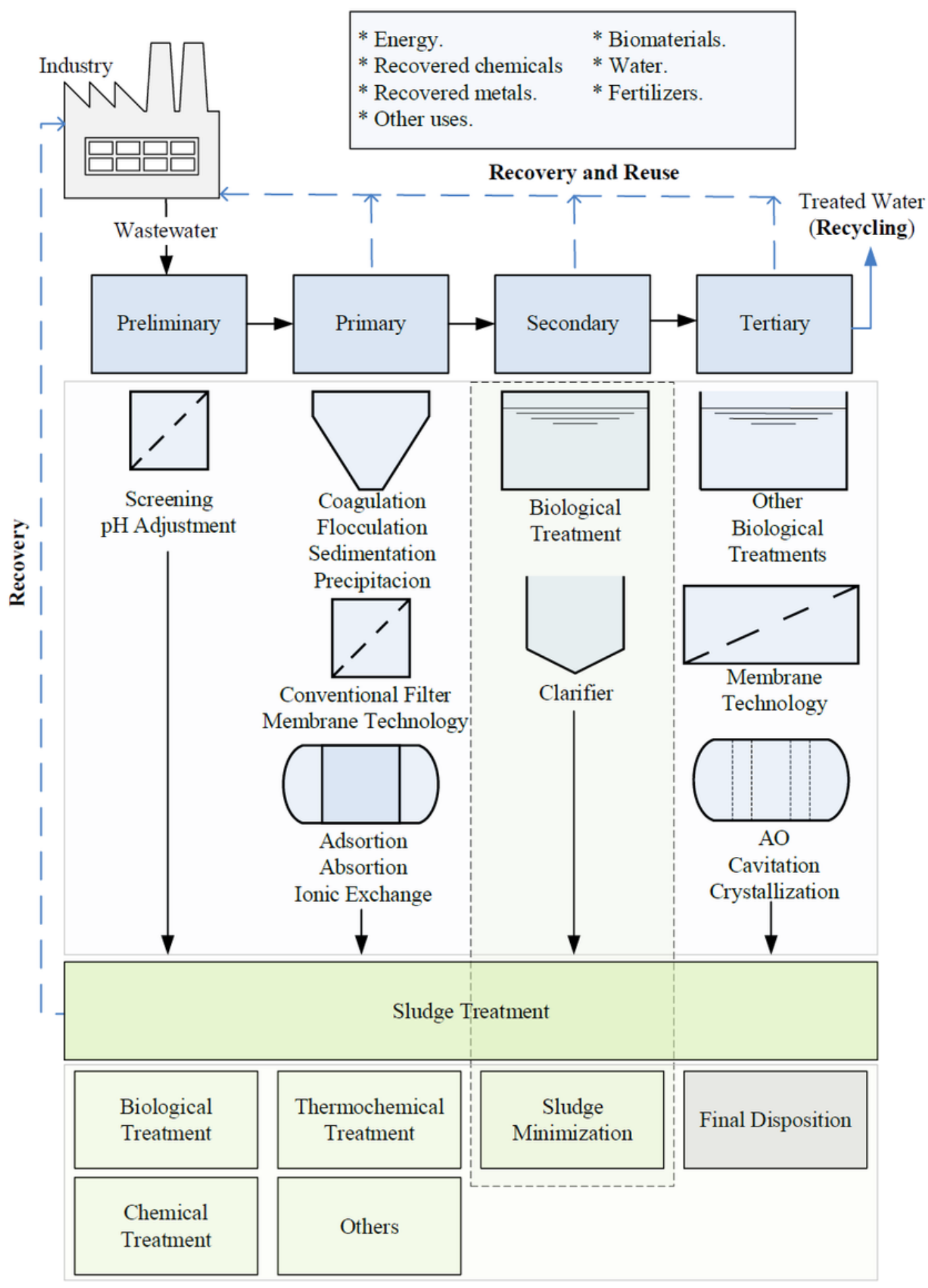

Figure 3

General combinations of unit operations and processes for a treatment system with recovery and revaluation of water and other contained elements. 


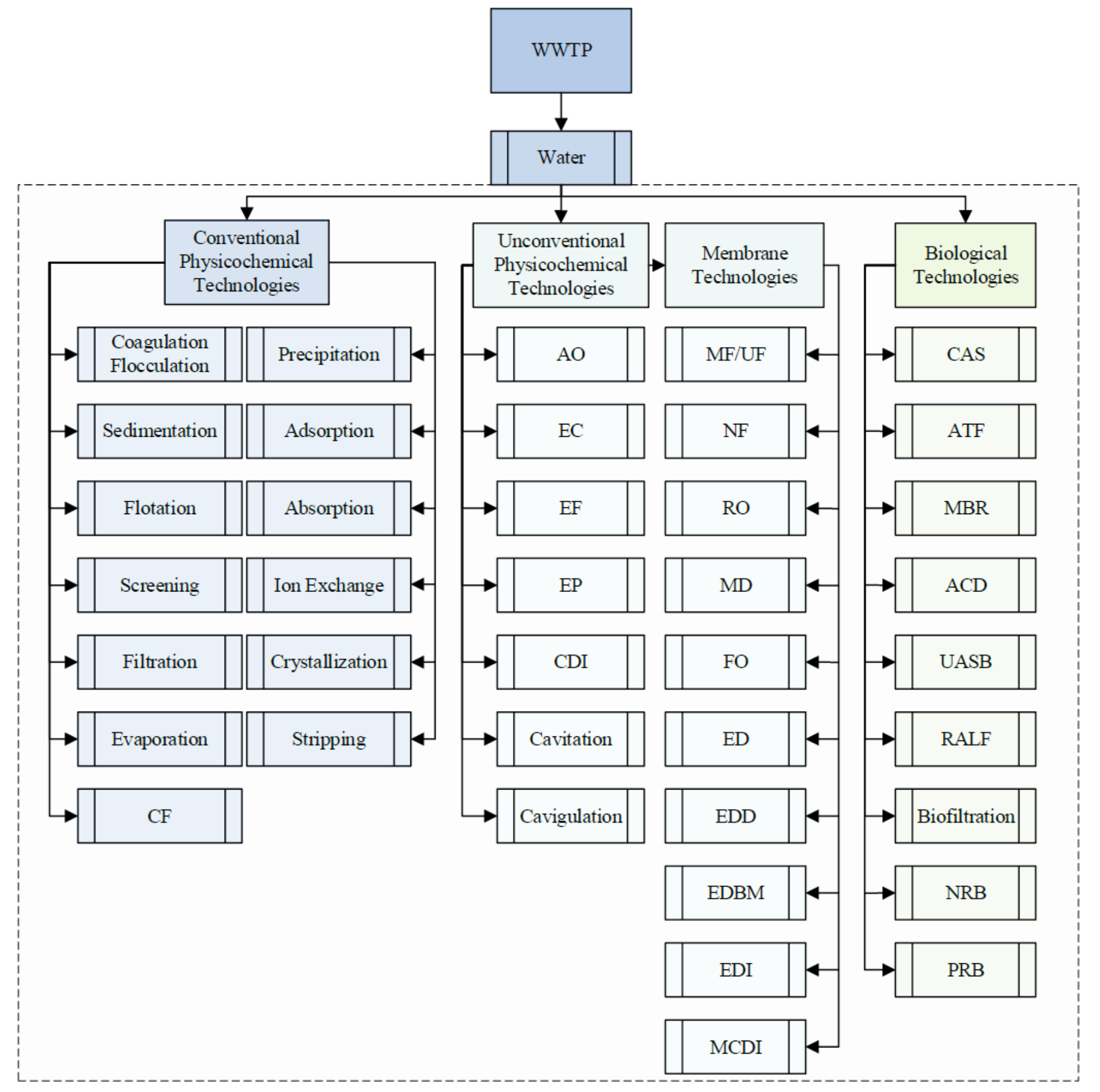

Figure 4

Wastewater treatment technologies. 


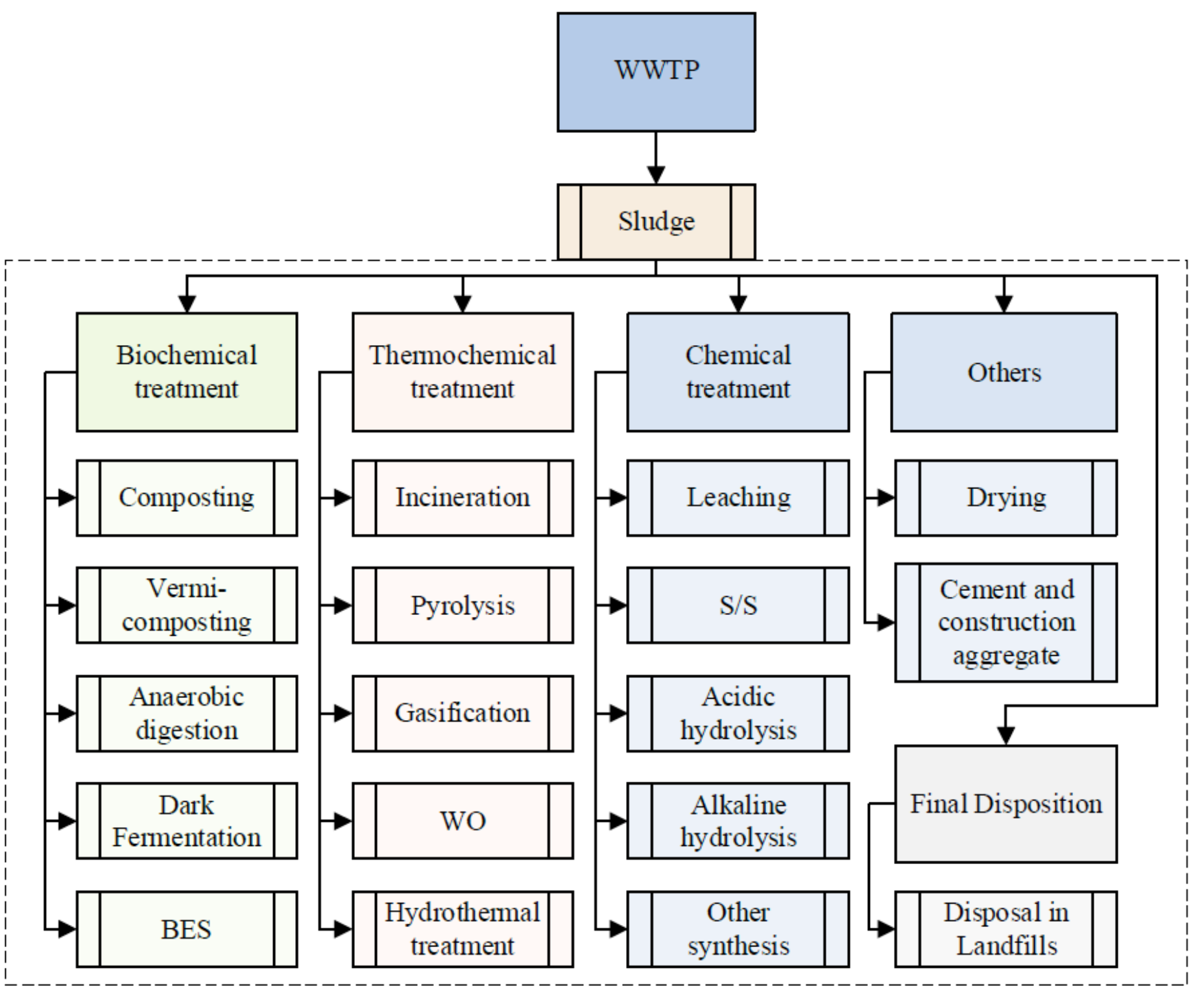

Figure 5

Derived sludge treatment technologies. 


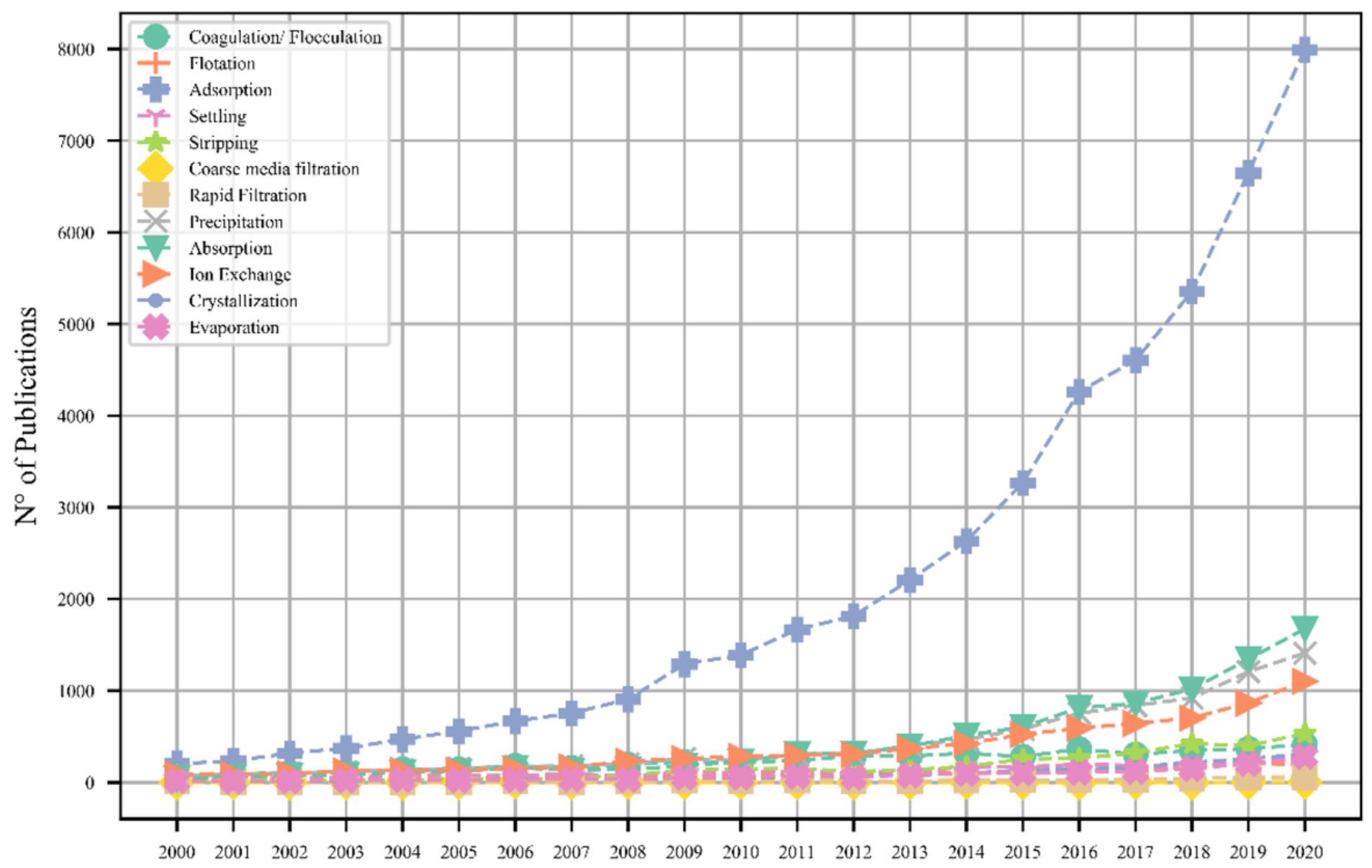

Figure 6

Number of publications of conventional wastewater treatment technologies.

Sources: Scopus by Elsevier. 

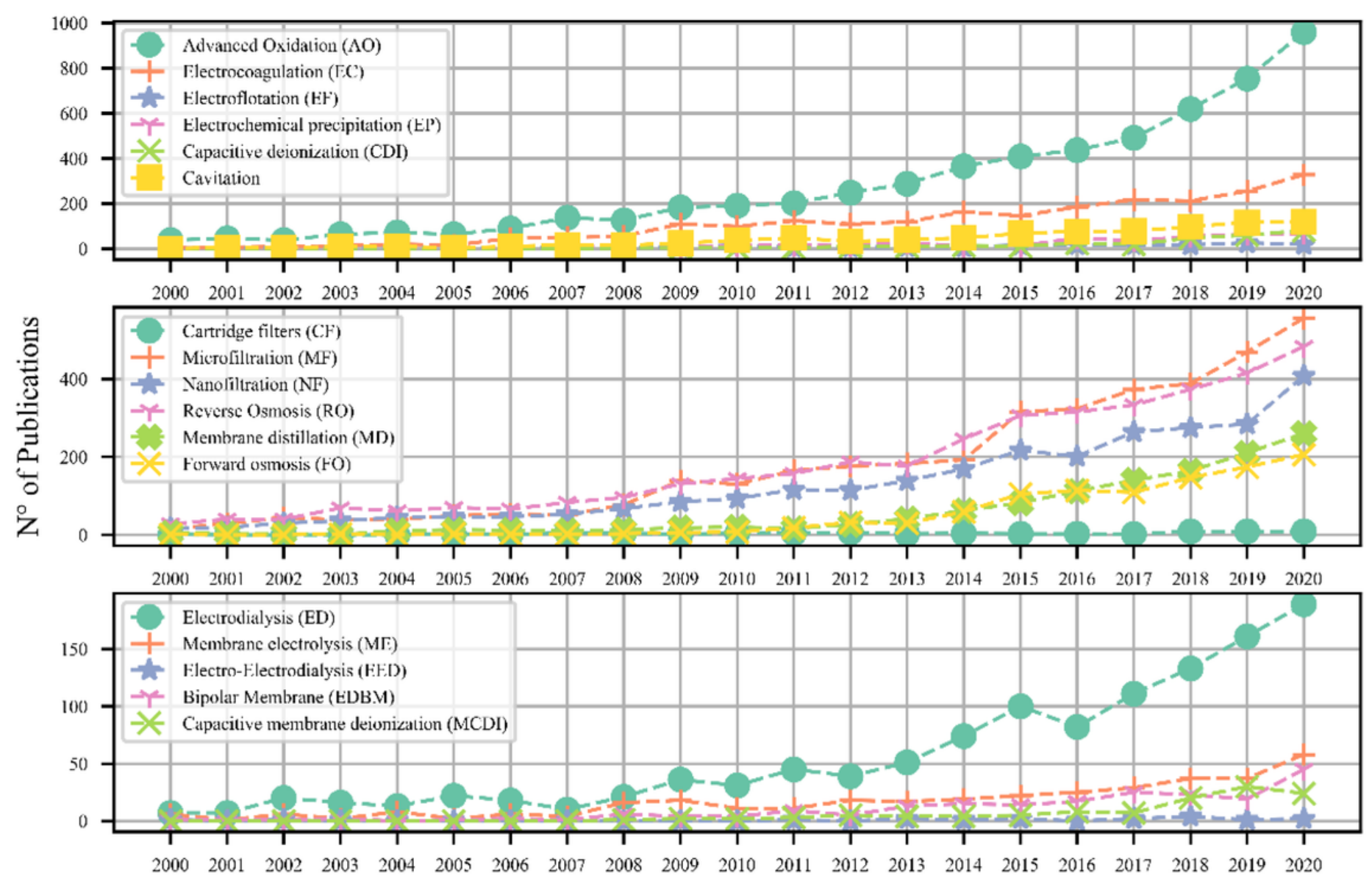

\section{Figure 7}

Number of publications of unconventional wastewater treatment technologies.

Sources: Scopus by Elsevier. 


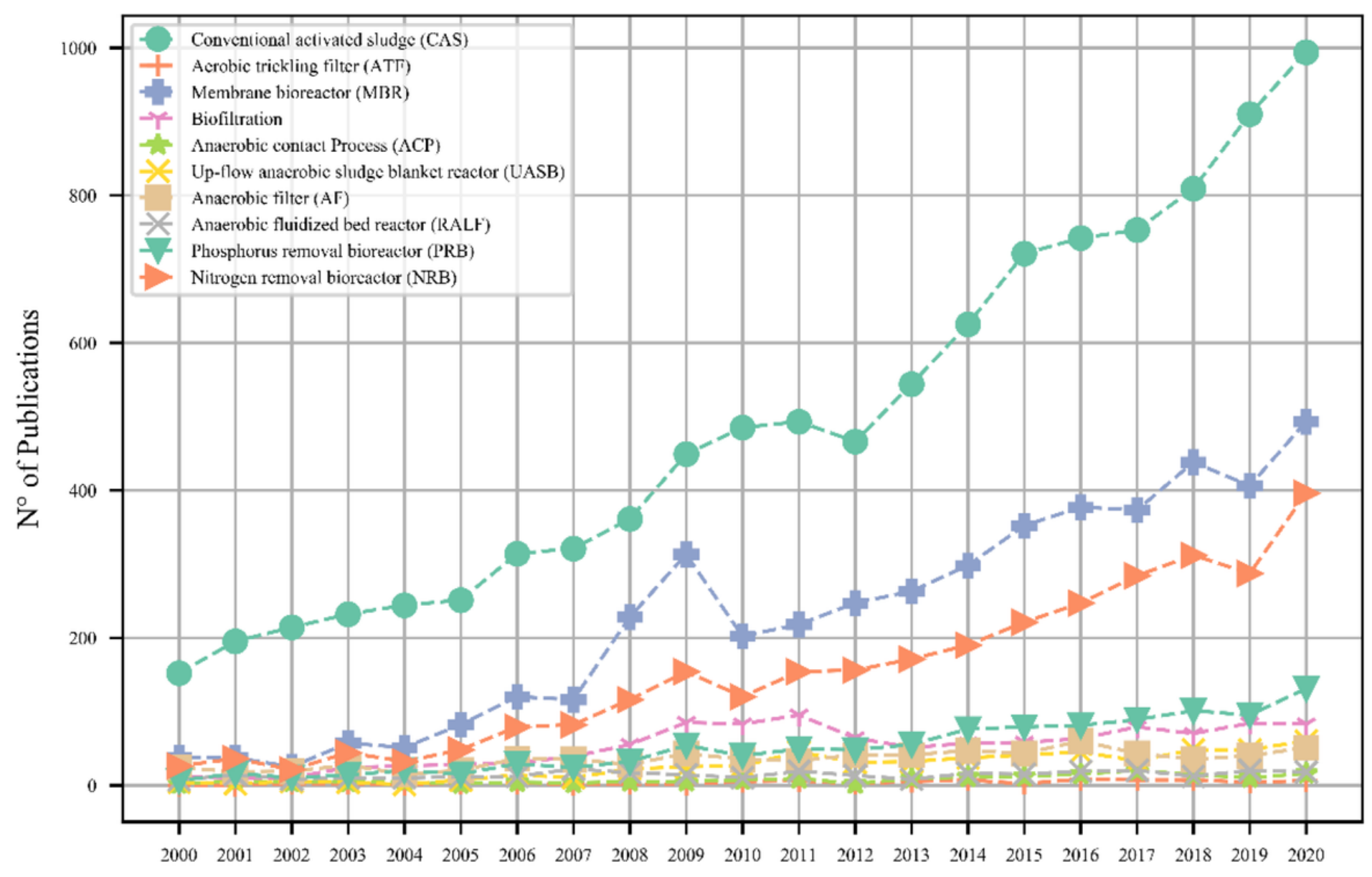

\section{Figure 8}

Number of publications of biological wastewater treatment technologies.

Sources: Scopus by Elsevier. 


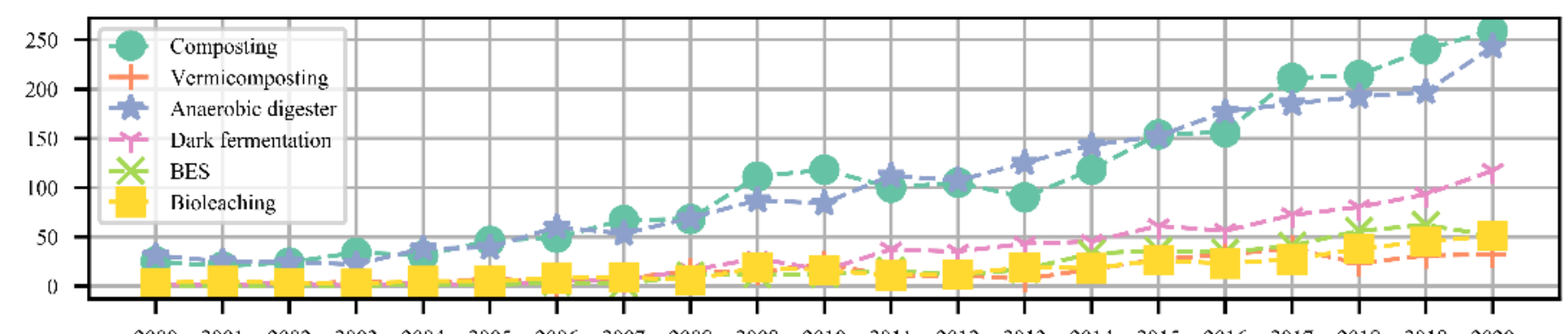

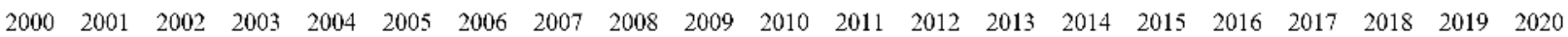

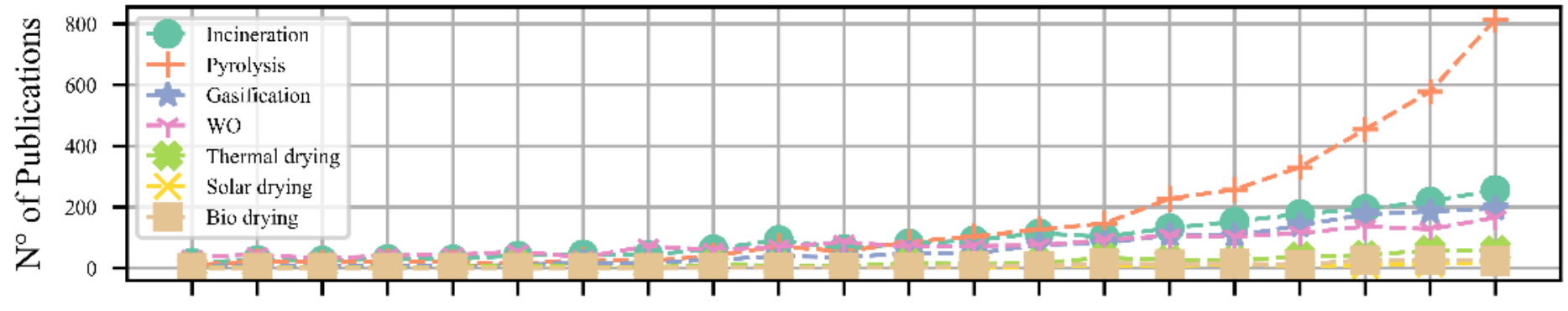

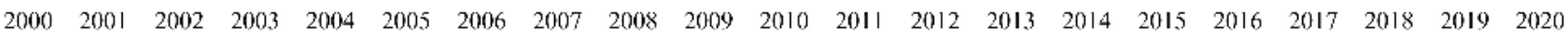

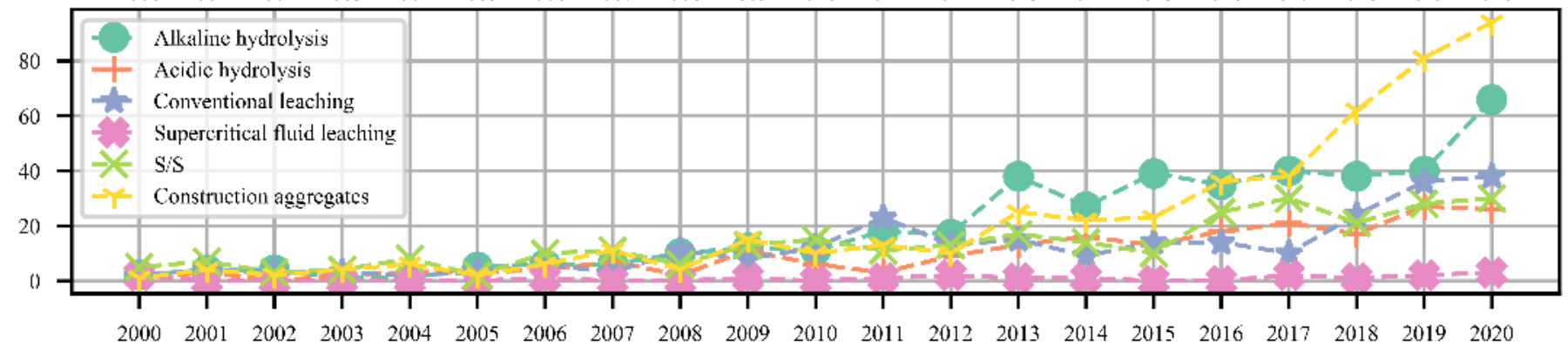

Figure 9

Number of publications of sludge from wastewater treatment technologies.

Sources: Scopus by Elsevier. 